\title{
SOLUTIONS OF NONLINEAR HYPERBOLIC EQUATIONS AT RESONANCE
}

\author{
L. CESARI* \\ Department of Mathematics, The University of Michigan, Ann Arbor, Michigan, U.S.A. \\ and \\ R. KANNAN $\dagger$ \\ Department of Mathematics, The University of Texas at Arlington, Arlington, Texas, U.S.A. \\ (Received 10 December 1980) \\ Key words and phrases: Operational equations, resonance, hyperbolic equations, condition (LL), \\ condition (*), Schauder's fixed point theorem, the spaces $\boldsymbol{A}_{p m}$, imbedding theorems, projection operators, \\ auxiliary equation, bifurcation equation, reduction to an alternative problem, wave equation.
}

\section{Introduction}

An Abstract Existence Analysis

2. Fixed Point Theorems

3. The Operational Equation

4. Preliminary Considerations Concerning the Hyperbolic Case

5. An Abstract Existence Theorem for the Hyperbolic Case

The Spaces $A_{p m}$

6. The spaces $A_{p m}$

7. Series Solutions for the Wave Equation

8. Complementary Remarks

9. A Measure Theoretical Property of Bounded Open Sets

10. Remarks on the (LL) and $\left(^{*}\right)$ conditions

\section{Applications}

11. Sufficient conditions for property $\left({ }^{*}\right)$

12. Analysis in the large of the equation $u_{f t}+u_{x x x x}=f(t, x, u)$

13. Estimation of $\omega_{0}$ for the elements of $A_{21}$

14. Analysis in the large of the doubly periodic solutions of the wave equation $u_{n}-u_{x x}=f(t, x, u)$

Appendix

\section{INTRODUCTION}

IN THE present paper we develop in some details an existence analysis for nonlinear abstract operator equations of the form

$$
E x=N x, \quad x \in X,
$$

particularly in view of applications to quasi-linear hyperbolic problems at resonance. Thus, if $E$ above is an unbounded linear operator $E: \operatorname{dom}(E) \rightarrow Y, \operatorname{dom}(E) \subset X$, where $X$ and $Y$ are real Banach spaces, and $N: X \rightarrow Y$ is a continuous nonnecessarily linear operator, we shall assume that the kernel of $E$ is not trivial and possibly infinite dimensional, $1 \leqslant \operatorname{dim} \operatorname{ker} E$

* Research partially supported by NSF award MCS 80-02337.

† Research partially supported by U.S. Army Research Grant DAAG29-80-C-0060. 
$\leqslant+\infty$, and that the partial inverse operator $H$ of $E$, or $H:$ Range $E \rightarrow X /$ ker $E$, is bounded, but not necessarily compact. Indeed, this is the difficult situation which actually may occur (dim $\operatorname{ker} E=\infty, H$ bounded but not necessarily compact) in hyperbolic problems. We shall call this the "hyperbolic case".

In this paper we shall see that the theorems we proved earlier (Cesari and Kannan [10, 11], Cesari $[5,6])$ for the "elliptic" case $(1 \leqslant \operatorname{dim} \operatorname{ker} E<+\infty, H$ compact $)$ have a natural extension to hyperbolic problems.

Of course, it may occur that for a given hyperbolic problem we have $\operatorname{dim} \operatorname{ker} E<\infty$ and $H$ compact. the latter may simply occur because of a suitable choice of spaces $X$ and $Y$ and their topologies. In this case, the theorems we proved earlier apply with no change.

Our analysis centers on suitable decompositions $X=X_{0} \times X_{1}, Y=Y_{0} \times Y_{1}$ of $X$ and $Y$, $X_{0}=\operatorname{ker} E, Y_{1}=$ range $E$, and the study of certain transformations $T: \Omega^{*} \rightarrow \Omega^{*}, \Omega^{*}=$ $S_{0} \times S_{1}, S_{0} \subset X_{0}, S_{1} \subset X_{1}$, or $\left(x^{*}, x_{1}\right) \rightarrow\left(\bar{x}^{*}, \bar{x}_{1}\right)$, of the form $\bar{x}_{1}=K_{1} x, \bar{x}^{*}=x^{*}-K_{0} x, x=$ $\left(x^{*}, x_{1}\right), \bar{x}=\left(\bar{x}^{*}, \bar{x}_{1}\right)$, so that their fixed points $x=\left(x^{*}, x_{1}\right), x=T x$, satisfy the equations $x_{1}=K_{1} x$ (auxiliary equation), and $K_{0} x=0$ (bifurcation equation). When needed, the map $T: \Omega^{*} \rightarrow \Omega^{*}$ is replaced by maps $T_{n}: \Omega_{n}^{*} \rightarrow \Omega_{n}^{*}, \Omega_{n}^{*}$ finite dimensional, in such a way that the sequence $\left[x_{n}\right]$ of fixed elements $x_{n}=T_{n} x_{n}$ is weakly convergent. The existence of at least a fixed point for $T$, (or for each $T_{n}$ ), is proved either by the Leray-Schauder topological argument, or by Schauder's fixed point theorem, based on the study of the inequality $\left(K_{0} x, x^{*}\right) \geqslant 0$ (or $\leqslant 0$ ) for $P x=x^{*}$.

In the elliptic case, as well as in ordinary differential equations, the inequality $\left(K_{0} x, x^{*}\right)$ $\geqslant 0$ (or $\leqslant 0$ ) (condition $(*)$ ) has been shown to include the Landesman and Lazer type conditions, and a number of other statements. We shall see the relevance of the same inequality in the hyperbolic case.

In Sections 2-5 we discuss some abstract theorems, in Sections 6-9 we summarize and briefly prove a number of statements concerning certain classes of Sobolev-type periodic functions and the Fourier series. In Section 10 we compare condition (*) with LandesmanLazer type conditions.

In Sections 11-14 we show that our uniform approach applies to problems in the large which had been previously discussed by Petzeltova, Hall, and others only in the perturbation case.

In [7] we shall see that a direct application of Schauder's fixed point theorem enables us to prove existence for some hyperbolic problems with $\infty$-dimensional kernel.

Some points of this paper have been presented at the May 1978 Conference in Florence, Italy, and distributed to the audience there in provisional form, and more points have been presented at the October 1979 Conference at Oklahoma State University, Stillwater (Differential Equations, pp. 1-21, Academic Press, New York, 1980).

\section{AN ABSTRACT EXISTENCE ANALYSIS}

\section{FIXED POINT THEOREMS}

Let $X=X_{0}+X_{1}$ be a decomposition of a real Hilbert space $X$, with inner product ( , ) norm \|\| , and projection operator $P: X \rightarrow X$ such that $P X=X_{0},(I-P) X=X_{1}$.

(2.i) Let $X$ be a real Hilbert space, and $X_{0}$ finite dimensional. Let $R, r$ be positive numbers, let $S_{0}=\left[x^{*} \in X_{0} \mid\left\|x^{*}\right\| \leqslant R\right], S_{1}=\left[x_{1} \in X_{1} \mid\left\|x_{1}\right\| \leqslant r\right]$ and $\Omega=S_{0} \times S_{1}$. Let $K_{1}: \Omega \rightarrow X_{1}$ be a 
compact map, let $K_{0}: \Omega \rightarrow X_{0}$ be a continuous map, and assume that (a) $\left\|K_{1} x\right\| \leqslant r$ for all $x$ $\in \Omega$; (b) $\left(K_{0} x, x^{*}\right) \leqslant 0$ [or $\geqslant 0$ ] for all $x=x^{*}+x_{1},\left\|x^{*}\right\|=R,\left\|x_{1}\right\| \leqslant r$. Then, there is at least one point $x=x^{*}+x_{1} \in \Omega$ with $x_{1}=K_{1} x, K_{0} x=0$.

Proof. Assume that $\left(K_{0} x, x^{*}\right) \leqslant 0$ holds in (b). Let $T: \Omega \rightarrow X$ denote the map defined by $T x=P x+K_{1} x+K_{0} x$, and note that, if $x$ is a fixed point of $T$, or $x=T x$, then, by writing $x=P x+(I-P) x$, we derive $(I-P) x-K_{1} x=K_{0} x$, where $(I-P) x-K_{1} x \in X_{1}$ and $-K_{0} x \in X_{0}$. Hence $(I-P) x-K_{1} x=0, K_{0} x=0$, that is, $x_{1}=K_{1} x, K_{0} x=0$.

Now we note that $T$ is a compact map, since $K_{1}$ is compact, and $P+K_{0}$ has finite dimensional range $X_{0}$. Thus, by the theory of Leray and Schauder, to prove that $T$ has some fixed point in $\Omega$, it is enough to prove that $(I-\lambda T) x \neq 0$ for all $x \in \Omega$ and $0<\lambda<1$. Indeed, for $x=$ $x^{*}+x_{1},\left\|x_{1} 1\right\|=r,\left\|x^{*}\right\| \leqslant R$ we have

$$
\begin{gathered}
\left((I-\lambda T) x, x_{1}\right)=\left\|x_{1}\right\|^{2}-\left(\lambda K_{1} x, x_{1}\right) \geqslant\left\|x_{1}\right\|^{2}-\lambda\left\|K_{1} x\right\|\left\|x_{1}\right\| \\
\\
\geqslant r^{2}-\lambda r^{2}>0 .
\end{gathered}
$$

For $x=x^{*}+x_{1},\left\|x_{1}\right\| \leqslant r,\left\|x^{*}\right\|=R$ we have analogously

$$
\left((I-\lambda T) x, x^{*}\right)=\left\|x^{*}\right\|^{2}-\lambda\left\|x^{*}\right\|^{2}-\lambda\left(K_{0} x, x^{*}\right)>0 .
$$

In any case $(I-\lambda T) x \neq 0$ for $x \in \partial \Omega, 0<\lambda<1$.

A statement similar to (2.i) was proved by Cesari and Kannan [10] by a different proof based on Schauder's fixed point theorem. Statement (2.i) was proved by Kannan and McKenna [19] by the argument given above. For extensions of (2.i) to Banach spaces, again based on Schauder's fixed point theorem, see Cesari $[5,6]$. Here is another version of $(2 . i)$ for Banach spaces, based on Schauder's fixed point theorem, and whose proof is particularly elementary and transparent.

Let us assume that there is a bilinear operator $X_{0} \times X_{0} \rightarrow$ reals, or $\langle u, v\rangle$, such that

$$
|\langle u, v\rangle| \leqslant\|u\|\|v\| \quad \text { for all } u, v \in X_{0} \text {; }
$$

$$
\langle u, u\rangle \geqslant 0 \text { for all } u, \text { and }\langle u, u\rangle=0 \text { if and only if } u=0 .
$$

If $X$ is a real Hilbert space then we can take for $\langle$,$\rangle the inner product. The existence of such$ operators $\langle u, v\rangle$ is a rather common occurrence (cf. Cesari [4]). Obviously, the linear operator $\langle u, v\rangle$ is continuous as an operator $X_{0} \times X_{0} \rightarrow$ reals.

Let $X=X_{0} \times X_{1}$ be a decomposition of a real Banach space $X$ with projection operator $P: X \rightarrow X$ such that $P X=X_{0},(I-P) X=X_{1}$. Here, by a projection operator $P$ we mean any linear bounded idempotent operator, and thus $X_{0}$ and $X_{1}$ are necessarily closed subspaces of the Banach space $X$ in the topology generated by the norm \|\| of $X$.

Let $R_{0}, r$ be positive numbers, let $S_{0}=\left[x^{*} \in X_{0} \mid\left\|x^{*}\right\| \leqslant R_{0}\right], S_{1}=\left[x_{1} \in X_{1}\left\|x_{1}\right\| \leqslant r\right]$, and $\Omega$ $=S_{0} \times S_{1}$.

(2.ii) Let $X$ be a real Banach space, let $X=X_{0}+\mathrm{X}_{1}$ be a decomposition of $X$ into closed subspaces of which $X_{0}$ is finite dimensional, and $P, R_{0}>0, r>0, S_{0}, S_{1}, \Omega$ be as above. Let $K_{0}: \Omega \rightarrow X_{0}$ be a continuous map, $K_{1}: \Omega \rightarrow X_{1}$ be a compact continuous map, and assume that $\left\|K_{0} x\right\| \leqslant J_{0},\left\|K_{1} x\right\| \leqslant J_{1}$ for all $x \in \Omega, J_{0}, J_{1}$ constants, with $J_{1} \leqslant r, J_{0}<R_{0}$. Let us also assume that $\left\langle K_{0} x, x^{*}\right\rangle \leqslant 0$ [or $\geqslant 0$ ] for all $x \in \Omega, x^{*}=P x, \bar{R}_{0} \leqslant\left\|x^{*}\right\| \leqslant R_{0}$, for some $\bar{R}_{0}<$ $R_{0}-J_{0}$. Then there is at least one point $x \in \Omega, x=x^{*}+x_{1}$, with $x_{1}=K_{1} x, K_{0} x=0$. 
Proof of (2.ii). Let us assume we always have $\left\langle K_{0} x, x^{*}\right\rangle \leqslant 0$. We take now positive numbers $R_{1}, R_{2}, R, \alpha, \beta$ satisfying the relations

$$
\begin{aligned}
& \bar{R}_{0} \leqslant R_{1}<R_{2}<R \leqslant R_{0}, \quad \alpha>0, \quad \beta>0, \\
& R_{1}+J_{0} \leqslant R, \quad R_{2}+J_{0} \leqslant R, \quad \alpha J_{0} R+\beta J_{0} \leqslant 2,
\end{aligned}
$$

which we shall prove below to be compatible with the hypotheses of the theorem. Let us consider the transformation $T: x \rightarrow \bar{x}$, or $\Omega \rightarrow X$, defined by

where

$$
T: \bar{x}_{1}=K_{1} x, \quad \bar{x}^{*}=x^{*}+g\left(x^{*}, x_{1}\right), \quad x=\left(x^{*}, x_{1}\right) \in \Omega, \quad \bar{x}=\left(\bar{x}^{*}, \bar{x}_{1}\right),
$$

and

$$
\begin{aligned}
& g\left(x^{*}, x_{1}\right)=K_{0} x \quad \text { for }\left\|x^{*}\right\| \leqslant R_{1}, \\
& g\left(x^{*}, x_{1}\right)=\left[\alpha\left\langle K_{0} x, x^{*}\right\rangle-\beta\left\|K_{0} x \mid\right\| x^{*} \text { for } R_{2} \leqslant\left\|x^{*}\right\| \leqslant R,\right. \\
& g\left(x^{*}, x_{1}\right)=\lambda K_{0} x+(1-\lambda)\left[\alpha\left\langle K_{0} x, x^{*}\right\rangle-\beta\left\|K_{0} x\right\| x^{*} \text { for } R_{1} \leqslant\left\|x^{*}\right\| \leqslant R_{2},\right.
\end{aligned}
$$

$$
\lambda=\left(R_{2}-R_{1}\right)^{-1}\left(R_{2}-\left\|x^{*}\right\|\right), \quad 0 \leqslant \lambda \leqslant 1 .
$$

Let us prove that $T$ maps $\Omega$ into itself. First, we note that $\left\|\bar{x}_{1}\right\|=\left\|K_{1} x\right\| \leqslant J_{1} \leqslant r$ in any case.

Now we note that, for $\left\|x^{*}\right\| \leqslant R_{1}$, we have $\bar{x}^{*}=x^{*}+K_{0} x$, hence

$$
\left\|\bar{x}^{*}\right\| \leqslant\left\|x^{*}\right\|+\left\|K_{0} x\right\| \leqslant R_{1}+J_{0} \leqslant R .
$$

For $R_{2} \leqslant\left\|x^{*}\right\| \leqslant R$, we have

$$
\begin{aligned}
& \alpha J_{0} R+\beta J_{0} \leqslant 2, \quad \bar{x}^{*}=\left[1+\alpha\left\langle K_{0} x, x^{*}\right\rangle-\beta\left\|K_{0} x \mid\right\| x^{*},\right. \\
& -1 \leqslant 1-\alpha J_{0} R-\beta J_{0} \leqslant 1+\alpha\left\langle K_{0} x, x^{*}\right\rangle-\beta\left\|K_{0} x\right\| \leqslant 1,
\end{aligned}
$$

and again $\left\|x^{*}\right\| \leqslant\left\|x^{*}\right\|$.

For $R_{1} \leqslant\left\|x^{*}\right\| \leqslant \mathrm{R}_{2}$, we have

$$
\bar{x}^{*}=\left[1+(1-\lambda)\left(\alpha\left\langle K_{0} x, x^{*}\right\rangle-\beta\left\|K_{0} x\right\|\right)\right] x^{*}+\lambda K_{0} x,
$$

where again the bracket is between -1 and 1 , and $\left\|\lambda K_{0} x\right\| \leqslant J_{0}$. Hence, $\left\|\bar{x}^{*}\right\| \leqslant$ $\left\|x^{*}\right\|+J_{0} \leqslant R_{2}+J_{0} \leqslant R$. We have proved that $T: \Omega \rightarrow \Omega$.

Now we have to prove that we have $\bar{x}^{*}=x^{*}$ if and only if $K_{0} x=0$. Certainly, $\bar{x}^{*}=x^{*}$ if and only if $g=0$, and, for $\left\|x^{*}\right\| \leqslant R_{1}$, certainly $g=0$ if and only if $K_{0} x=0$.

For $R_{2} \leqslant\left\|x^{*}\right\| \leqslant R$, we have

$$
g=\left[\alpha\left\langle K_{0} x, x^{*}\right\rangle-\beta\left\|K_{0} x\right\|\right] x^{*}, \quad x^{*} \neq 0,
$$

and $\alpha\left\langle K_{0} x, x^{*}\right\rangle-\beta\left\|K_{0} x\right\| \leqslant-\beta\left\|K_{0} x\right\|$. Thus, $g=0$ if and only if $\left\|K_{0} x\right\|=0$. For $R_{1}<\left\|x^{*}\right\|<$ $R_{2}$, we have

hence

$$
g=\lambda K_{0} x+(1-\lambda)\left[\alpha\left\langle K_{0} x, x^{*}\right\rangle-\beta\left\|K_{0} x\right\|\right] x^{*},
$$

$$
\left\langle g, x^{*}\right\rangle=\lambda\left\langle K_{0} x, x^{*}\right\rangle+(1-\lambda)\left[\alpha\left\langle K_{0} x, x^{*}\right\rangle-\beta\left\|K_{0} x\right\|\right]\left\langle x^{*}, x^{*}\right\rangle,
$$

where now $\lambda>0,1-\lambda>0,\left\langle K_{0} x, x^{*}\right\rangle \leqslant 0, x^{*} \neq 0$, hence $\left\langle x^{*}, x^{*}\right\rangle>0$. Thus, $\left\langle g, x^{*}\right\rangle<0$ for $K_{0} x \neq 0$, and finally $g=0$ if and only if $\left\|K_{0} x\right\|=0$.

In any case, that is, for any $x^{*},\left\|x^{*}\right\| \leqslant R$, we have $\bar{x}^{*}=x^{*}$ if and only if $K_{0} x=0$. 
Here, $K_{1}$ is compact by hypothesis, and $K_{0}$ is continuous and bounded, and has finite dimensional range. Thus, $T: \Omega \rightarrow \Omega$ is a continuous compact map and $\Omega$ is closed and convex. By Schauder's fixed point theorem, there is a fixed point $x=T x, x=\left(x^{*}, x_{1}\right) \in \Omega$, and then $x_{1}=K_{1} x$ and $\bar{x}^{*}=x^{*}$, hence $K_{0} x=0$.

Relations (2.3) are compatible with the hypotheses of the theorem. Indeed, $R_{0}-J_{0}>\bar{R}_{0}$ and we can take for instance $R=R_{0}, R_{2}=R_{0}-J_{0}$, and $R_{1}$ any number $\bar{R}_{0}<R_{1}<R_{0}-$ $J_{0}$. Finally, we can choose arbitrary numbers $\alpha>0, \beta>0$, sufficiently small, so that $\alpha R_{0} J_{0}+\beta J_{0} \leqslant 2$.

Remark 1. Instead of considering the transformation $T$ defined by (2.4-2.5), we could have considered the transformation $T$ defined by

$$
\bar{T}: \bar{x}_{1}=K_{1} x, \quad \bar{x}^{*}=x^{*}+g\left(x^{*}+\bar{x}_{1}, x^{*}\right),
$$

where as before $x=x^{*}+x_{1}, \bar{x}=\bar{x}^{*}+\bar{x}_{1}, x^{*}, \bar{x}^{*} \in X_{0}, \quad x_{1}, \bar{x}_{1} \in X_{1},\left\|x^{*}\right\| \leqslant R_{0},\left\|x_{1}\right\| \leqslant r$, and where $P \bar{x}=\bar{x}^{*}$. This transformation has been already used in (Cesari [6]).

Remark 2. It is clear that the inequality $\left(K_{0} x, x^{*}\right) \leqslant 0$ [or $\geqslant 0$ ] is only a devise to guarantee that $T: \bar{x}_{1}^{*}=K_{1} x, \bar{x}^{*}=x^{*}-K_{0} x, x \in \Omega$, maps $\Omega$ into itsclf with $I-\lambda T$ having a constant topological degree as $\lambda$ describes $[0,1]$ and hence-under compactness hypotheses and by Leray-Schauder's theory- $T$ has a fixed point in $\Omega$. Often, we shall be able to prove directly that $T$ maps $\Omega$ into itself, and then the existence of a fixed point under the same compactness hypotheses follow from Schauder's fixed point theorem.

\section{THE OPERATIONAL EQUATION}

Let $X, Y$ be real Hilbert spaces. Let $E: D(E) \rightarrow Y$ be a linear operator with domain $D(E)$ $\subset X$, let $N: X \rightarrow Y$ be an operator nonnecessarily linear, and let us consider the equation

$$
E x=N x, \quad x \in X
$$

Let ker $E$ denote the kernel of $E$, that is, the subspace of $X$ of all $x \in X$ with $E x=0$, and let $Y_{1}$ denote the range of $E$. Let us assume that there are projection operators $P: X \rightarrow X$ and $Q: Y \rightarrow Y$ such that

$$
\begin{aligned}
& P X=X_{0} \supset \operatorname{ker} E, \quad(I-P) X=X_{1}, \\
& Q Y=Y_{0}, \quad(I-Q) Y=Y_{1}=\text { range } E .
\end{aligned}
$$

The map $E: D(E) \cap X_{1} \rightarrow Y_{1}$ is one-one and onto, and the inverse map $H: Y_{1} \rightarrow D(E) \cap$ $X_{1}$ is, therefore, one-one and onto, and $H$ is linear. We need only to assume that $E, H, P$, $Q$ satisfy the relations (a) $H(I-Q) E x=(I-P) x$, (b) $Q E x=E P x$, (c) $E H(I-Q) x=$ $(I-Q) x$. Then, it is easy to verify that equation (3.1) is equivalent to the following system of auxiliary and bifurcation equations

$$
\begin{gathered}
x=P x+H(I-Q) N x, \\
Q(E-N) x=0 .
\end{gathered}
$$

If $X_{0}=\operatorname{ker} E$, then (b) reduces to $Q E x=E P x=0$, and by writing $x=x^{*}+x_{1}, x^{*}=P x$ $\in X_{0}, x_{1}=(I-P) x \in X_{1}$, auxiliary and bifurcation equations reduce to

$$
x=H(I-Q) N\left(x^{*}+x_{1}\right)
$$




$$
Q N\left(x^{*}+x_{1}\right)=0
$$

Let $L=\|H\|$. Also, let $S: Y_{0} \rightarrow X_{0}$ by any continuous operator for which we only require here that $S^{-1}(0)=0$. Then, equation (3.3) can be replaced by $S Q(E-N) x=0$ and equation (3.5) by $S Q N x=0$. Moreover, equation (3.3) can be replaced by $S(E P-Q N) x=0$.

(3.i) (An abstract theorem for the case $\operatorname{dim} \operatorname{ker} E<\infty$ and $H$ compact).

Let $X_{0}=\operatorname{ker} E$ be nontrivial and finite dimensional, let $H$ be compact, let $N$ and $S$ be continuous operators. Let us assume that there are numbers $R, r$ positive such that (a) for all $x^{*} \in X_{0}, x_{1} \in X_{1},\left\|x^{*}\right\| \leqslant R,\left\|x_{1}\right\| \leqslant r$ we have $\left\|N\left(x^{*}+x_{1}\right)\right\| \leqslant L^{-1} r$, (b) for all $x^{*} \in X_{0}, x_{1} \in$ $X_{1},\left\|x^{*}\right\|=R,\left\|x_{1}\right\| \leqslant r$ we have $\left(\operatorname{SQN}\left(x^{*}+x_{1}\right), x^{*}\right) \geqslant 0$ [or $\leqslant 0$ ], then equation $E x=N x$ has at least one solution $x=x^{*}+x_{1}, x^{*} \in X_{0}, x_{1} \in X_{1},\left\|x^{*}\right\| \leqslant R,\left\|x_{1}\right\| \leqslant r,\|x\| \leqslant\left(R^{2}+r^{2}\right)^{1 / 2}$.

We need only apply (2.i) with $K_{1} x=H(I-Q) N x$ and $K_{0} x=S Q N x$.

Remark. If $X_{0} \supset$ ker $E$, if $X_{0}$ is finite dimensional, and we assume $E$ to be continuous on $X_{0}$, then both $S E P$ and $S Q N$ are continuous maps on $X$, and (3.i) still holds with the inequality in (b) replaced by $\left(S(E P-Q N)\left(x^{*}+x_{1}\right), x^{*}\right) \geqslant 0$ [or $\left.\leqslant 0\right]$. The proof is the same.

The following corollaries for $X=Y, P=Q, S=I$, are worth noting.

COROLlaRY 1. If we know that there are constants $J_{0}>0, K \geqslant L J_{0}$ such that (A) $\|N x\| \leqslant J_{0}$ for all $x \in X$; (B) $\left(N\left(x^{*}+x_{1}\right), x^{*}\right) \geqslant 0 \quad$ [or $\left.\leqslant 0\right]$ for all $x^{*} \in X_{0}, x_{1} \in X_{1}$, with $\left\|x^{*}\right\| \leqslant R_{0},\left\|x_{1}\right\| \leqslant K$, then conditions (a), (b) of (3.i) hold.

Indeed we can take any $R \geqslant R_{0}$ and $r=K$, so that $\|N x\| \leqslant J_{0}=L^{-1}\left(L J_{0}\right) \leqslant L^{-1} K=L^{-1} r$.

CoROLlaRY 2. If we know that there are constants $J_{0} \geqslant 0, J_{1}>0,0<k<1, R_{0} \geqslant 0, K_{0} \geqslant$ $L J_{0}, K_{1}>L J_{1}$ such that:

$\left(A_{k}\right) \quad\|N x\| \leqslant J_{0}+J_{1}\|x\|^{k}$ for all $x \in X$;

$\left(B_{k}\right) \quad\left(N\left(x^{*}+x_{1}\right), x^{*}\right) \geqslant 0[$ or $\leqslant 0]$ for all $x^{*} \in X_{0}, x_{1} \in X_{1}$

with $\left\|x^{*}\right\| \geqslant R_{0},\left\|x_{1}\right\| \leqslant K_{0}+K_{1}\|x\|^{k}$;

then conditions (a), (b) of (3.i) hold.

Indeed, first choose a constant $\alpha>0$ such that $J_{0}+J_{1}\left(1+\alpha^{2}\right)^{k / 2} R_{0}^{k} \geqslant L^{-1} \alpha R_{0}$ and $L J_{1}\left(1+\alpha^{2}\right)^{k / 2} \leqslant K_{1}$, then take $r=\alpha R$ and take $R \geqslant R_{0}$ so that $J_{0}+J_{1}\left(1+\alpha^{2}\right)^{k / 2} R^{k}=$ $L^{-2} \alpha R$. Now for $\left\|x_{1}\right\| \leqslant r,\left\|x^{*}\right\| \leqslant R$, we have

$$
L\|N x\| \leqslant L\left(J_{0}+J_{1}\left(1+\alpha^{2}\right)^{k / 2} R^{k}\right)=\alpha R=r .
$$

On the other hand, for $\left\|x_{1}\right\| \leqslant r,\left\|x^{*}\right\|=R$, we also have

$$
\begin{aligned}
\left\|x_{1}\right\| \leqslant r & =\alpha R=L\left(J_{0}+J_{1}\left(1+\alpha^{2}\right)^{k / 2} R^{k}\right) \\
& \leqslant K_{0}+\left(L J_{1}\right)\left(1+\alpha^{2}\right)^{k / 2} R^{k} \leqslant K_{0}+K_{1} R^{k} \leqslant K_{0}+K_{1}\|x\|^{k} .
\end{aligned}
$$


COROLlaRY 3. If we know that there are constants $J_{0} \geqslant 0, J_{1}>0, k \geqslant 1, R_{0} \geqslant 0, K_{0}>$ $L J_{0}, K_{0}>L J_{1}$, such that $\left(A_{k}\right),\left(B_{k}\right)$ hold (for fixed $k \geqslant 1$ ), and $\left(C_{k}\right) J_{1} \in \gamma$ where $\gamma>0$ is a constant which depends only on $R_{0}, L, K_{1}$, then the conditions (a), (b) of (3.i) hold.

The proof is similar to the one for $0<k<1$. First we choose $\alpha>0$ so that $L J_{1}\left(1+\alpha^{2}\right)^{k / 2}=K_{1}$, hence $\alpha=\left(\left(K_{1} L^{-1} J_{1}^{-1}\right)^{2 / k}-1\right)^{1 / 2}$. Now we take $r=\alpha R$ and $R \geqslant R_{0}$ so as to satisfy

$$
J_{0}+J_{1}\left(1+\alpha^{2}\right)^{k / 2} R^{k}=L^{-1} \alpha R .
$$

If we can find such an $R \geqslant R_{0}$ the argument is the same as before. To verify that such an $R \geqslant R_{0}$ exists we write the equation for $R$ in the form

$$
J_{0} R^{-1}+J_{1}\left(K_{1} L^{-1} J_{1}^{-1}\right) R^{k-1}=L^{-1}\left(\left(K_{1} L^{-1} J_{1}^{-1}\right)^{2 / k}-1\right)^{1 / 2},
$$

where $k-1 \geqslant 0$. Thus, all we have to require is that $J_{0}>0$ is sufficiently small, namely so that

$$
J_{0} R_{0}^{-1}+K_{1} L^{-1} R_{0}^{k-1}<L^{-1}\left(\left(K_{1} L^{-1} J_{1}^{-1}\right)^{2 / k}-1\right)^{1 / 2} .
$$

\section{PRELIMINARY CONSIDERATIONS CONCERNING THE HYPERBOLIC CASE}

Let $E, N$ be operators from their domains $D(E), D(N)$ in a space $\mathscr{D}$ with ranges in a space $\mathscr{Y}$, both $\mathscr{X}$ and $\mathscr{Y}$ real Banach spaces or Hilbert spaces. Let us consider the operator equation $E x=N x$ as in Section 2. Its solution $x$ in $\mathscr{X}$ may be expected to be usual solutions, or generalized solutions, according to the choice of $\mathscr{X}$. We shall consider first smaller spaces $X$ and $Y$, say $X \subset \mathscr{X}, Y \subset \mathscr{Y}$, both real Hilbert spaces, and we shall assume that the inclusion map $j: X \rightarrow \mathscr{X}$ is compact.

We shall then construct a sequence of elements $\left[x_{k}\right], x_{k} \in X$, which is bounded in $X$, or $\left\|x_{k}\right\| \leqslant M$. Then, there is a subsequence, say still $[k]$ for the sake of simplicity, such that $\left[j x_{k}\right]$ converges strongly in $\mathscr{X}$ toward some element $\xi$. On the other hand, $X$ is Hilbert, hence reflexive, and we can take the subsequence, say still $[k]$, in such a way that $x_{k} \rightarrow x$ weakly in $X$. Actually, $\zeta=j x$, that is, $\zeta$ is the same element $x \in X$ thought of as an element in $\mathscr{X}$. In other words:

(4.i) If $x_{k} \rightarrow x$ weakly in $X$ and $j x_{k} \rightarrow \zeta$ strongly in $\mathscr{X}$, then $\zeta=j x$.

Indeed, $j: X \rightarrow \mathscr{X}$ is a linear compact map, hence continuous (see, e.g., [3, p. 285, Theorem 17.1]). As a consequence, $x_{k} \rightarrow x$ weakly in $X$ implies that $j x_{k} \rightarrow j x$ weakly in $\mathscr{X}$ (see, e.g., $\left[3\right.$, p. 295, Proposition 12]). Since $j x_{k} \rightarrow \zeta$ strongly in $\mathscr{X}$, we have $\zeta=j x$.

We shall assume that $X_{1}$ and $X_{0}$ contain finite dimensional subspaces $X_{1 n}, X_{0 n}$ such that $X_{1 n} \subset X_{1, n+1} \subset X_{1}, X_{0 n} \subset X_{0, n+1} \subset X_{0}, n=1,2, \ldots$, with $\cup_{n} X_{1 n}=X_{1}, \quad \cup_{n} X_{0 n}=X_{0}, \quad$ and assume that there are projection operators $R_{n}: X_{1} \rightarrow X_{1 n}, S_{n}: X_{0} \rightarrow X_{0 n}$ with $R_{n} X_{1}=X_{1 n}$, $S_{n} X_{0}=X_{0 n}$ (cf. similar assumptions in Rothe [23]). Since $X$ is a real Hilbert space, we may think of $R_{n}$ and $S_{n}$ as orthogonal projections and then $\left\|R_{n} x\right\|_{X} \leqslant\|x\|_{X},\left\|S_{n} x^{*}\right\|_{X} \leqslant\left\|x^{*}\right\|_{X}$ for all $x \in X_{1}$ and $x^{*} \in X_{0}$.

Thus, we see that in the process of limit just mentioned, $x_{k} \rightarrow x$ weakly in $X, j x_{k} \rightarrow j x$ strongly in $\mathscr{X}$, and the limit element can still be thought of as belonging to the smaller space $X$. This situation is well known in the important case $X=W_{2}^{N}(G), \mathscr{X}=W_{2}^{m}(G), 0 \leqslant n<N$, $X \subset \mathscr{X}, G$ any open set in some $\mathbb{R}^{\nu}, \nu \geqslant 1$. Then, the weak convergence $x_{k} \rightarrow x$ in $W_{2}^{N}(G)$ 
implies the strong convergence $j x_{k} \rightarrow j x$ in $W_{2}^{n}(G)$, and $\zeta=j x$ is still an element of the smaller space $X=W_{2}^{N}(G)$.

Concerning the subspaces $X_{0 n}$ of $X_{0}$ it is not restrictive to assume that there is a complete orthonormal system $\left[v_{1}, v_{2}, \ldots, v_{n}, \ldots\right]$ in $X_{0}$ and that $X_{0 n}=s p\left(v_{1}, v_{2}, \ldots, v_{n}\right), n=1,2$, .... We shall further assume that there is a complete orthonormal system ( $w_{1}$, $\left.w_{2}, \ldots, w_{n}, \ldots\right)$ in $Y_{0}$, we take $Y_{0 n}=s p\left(w_{1}, \ldots, w_{n}\right)$, and denote by $S_{n}^{\prime}$ the orthogonal projection of $Y_{0}$ onto $Y_{0 n}$.

We consider now the coupled system of operator equations

$$
\begin{gathered}
x=S_{n} P x+R_{n} H(I-Q) N x, \\
0=S_{n}^{\prime} Q N x .
\end{gathered}
$$

We shall now define a map $\alpha_{n}: Y_{0 n} \rightarrow X_{0 n}$ by taking $\alpha_{n} y=\Sigma_{1}^{n}\left(y, w_{i}\right) v_{i}$. Then, we have $0=$ $\alpha_{n} S_{n}^{\prime} Q N x$ if and only if $0=S_{n}^{\prime} Q N x$, that is, $\alpha_{n}^{-1} 0=0$. We conclude that system $(4.1-4.2)$ is equivalent to system

$$
\begin{gathered}
x=S_{n} P x+R_{n} H(I-Q) N x, \\
0=\alpha_{n} S_{n}^{\prime} Q N x .
\end{gathered}
$$

(4.ii) (LEMMA). Under the hypotheses above, let us assume that there are constants $R$, $r>0$ such that

(a) for all $x^{*} \in X_{0}, x_{1} \in X_{1},\left\|x^{*}\right\| \leqslant R$, $\left\|x_{1}\right\| \leqslant r$, we have $\left\|N\left(x^{*}+x_{1}\right)\right\| \leqslant L^{-1} r$;

(b) for all $\left\|x^{*}\right\|=R,\left\|x_{1}\right\| \leqslant r$ we have $\left(\alpha_{n} S_{n}^{\prime} Q N\left(x^{*}+x_{1}\right), x^{*}\right) \geqslant 0$ [or $\leqslant 0$ ].

Then, for every $n$, system (4.1), (4.2) has at least a solution $x_{n}=x_{0 n}^{*}+x_{1 n}, x_{n} \in D(E) \cap$ $\left(X_{0 n} \times X_{1 n}\right), S_{n} P x_{n}=x_{0 n}^{*}$, with $\left\|x_{n}\right\| \leqslant M=\left(R^{2}+r^{2}\right)^{1 / 2}, M$ independent of $n$.

Proof. If we consider the subset $C_{n}$ of $X_{0 n} \times X_{1 n}$ made up of all $x=x^{*}+x_{1}, x^{*} \in X_{0}, x_{1} \in$ $X_{1 n}$ with $\left\|x^{*}\right\| \leqslant R,\left\|x_{1}\right\| \leqslant r$, we see that

$$
\begin{gathered}
\left\|R_{n} H(I-Q) N x\right\| \leqslant r \text { for all } x \in C_{n}, \\
\left(\alpha_{n} S_{n}^{\prime} Q N x, x^{*}\right) \geqslant 0[\text { or } \leqslant 0] \quad \text { for all } x \in C_{n} \text { with }\left\|x^{*}\right\|=R .
\end{gathered}
$$

Now the assumptions actually used in the proof of (3.i) are satisfied. In particular, the compactness of the bounded operator $R_{n} H$ follows from the fact that $R_{n} H$ has a finite dimensional range, and the finite dimensionality of the kernel of $E$ is now replaced by the fact that the range of $\alpha_{n} S_{n}^{\prime} Q N$ is certainly finite dimensional. The bound $M=\left(R^{2}+r^{2}\right)^{1 / 2}$ is independent of $n$.

\section{AN ABSTRACT EXISTENCE THEOREM FOR THE HYPERBOLIC CASE}

In order to solve the equation $E x=N x$ we now adopt a "passage to the limit argument". We assume that both the Hilbert spaces $X$ and $Y$ are contained in the real Banach (or Hilbert) spaces $\mathscr{X}$ and $\mathscr{Y}$ with compact injections $j: X \rightarrow \mathscr{X}, j^{\prime}: Y \rightarrow \mathscr{Y}$. Actually, we can limit ourselves to the consideration of the spaces $\mathbb{P}$ and $\bar{Y}$ made up of limit elements from sequences in $X$ and $Y$ respectively as mentioned in Section 4. Hence, $\mathscr{X}$ is identical to $X$ and $\mathscr{Y}$ is identical to $Y$, though they may have different topologies. We shall write $\mathscr{X}=j X, \bar{Y}=j^{\prime} Y$. 
Analogously, we take $\mathscr{X}_{0}=j X_{0}, \mathscr{Y}_{0}=j^{\prime} Y_{0}, \mathscr{X}_{1}=j X_{1}, \mathscr{Y}_{1}=j^{\prime} Y_{1}$, and the linear operators $\bar{P}: \overline{\mathscr{X}} \rightarrow \mathscr{X}_{0}, Q: \overline{\mathscr{Y}} \rightarrow \mathscr{Y}_{0}$ are then defined by $P x=x^{*}$ in $\overline{\mathscr{X}}$ if $P x=x_{0}$ in $X ; Q y=y^{*}$ in $\mathscr{Y}$ if $Q y=y^{*}$ in $\bar{Q}$.

We now assume the following:

(c) $x_{n} \rightarrow x$ weakly in $X$ and $j x_{n} \rightarrow j x$ strongly in $\mathscr{X}$ implies that $N x_{n} \rightarrow N x$ strongly in $\mathscr{Y}, S_{n} P x_{n}$ $\rightarrow P x$ strongly in $\mathscr{Y}$, and $R_{n} x_{n} \rightarrow x$ strongly in $\mathscr{X}$.

Under the hypotheses of (4.ii) there are elements $x_{n} \in X_{n}$ such that

$$
\begin{gathered}
x_{n}=S_{n} P x_{n}+R_{n} H(I-Q) N x_{n}, \\
0=\alpha_{n} S_{n}^{\prime} Q N x_{n},
\end{gathered}
$$

where $\left\|x_{n}\right\| \leqslant M$ for all $n$. Hence, there exists a subsequence, say still $\left[x_{n}\right]$, such that $x_{n} \rightarrow$ $x$ weakly in $X$ and $j x_{n} \rightarrow j x$ strongly in $\mathscr{X}$. Then, by (5.1), (5.2), proceeding to the limit, we have

$$
x=P x+H(I-Q) N x, \quad 0=Q N x, \quad x \rightarrow \overline{\mathscr{X}} .
$$

Indeed, as $n \rightarrow \infty, S_{n}^{\prime}$ converges to the identity $I: Y_{0} \rightarrow Y_{0}$ and $\alpha_{n}$ converges to a homeomorphism $\alpha: Y_{0} \rightarrow X_{0}$ in the sense that $S_{n} y \rightarrow y, \alpha_{n} y \rightarrow y$ as $n \rightarrow \infty$.

We now remark that, in $\mathscr{X}$ the operator $E$ may have no meaning and thus the concept of solution of $E x=N x$ has to be properly understood. However, $x \in \mathscr{X}$ and thus, by Section $4, x$ is still an element of $X$ on which $E$ is defined. Furthermore, as a consequence of the hypotheses on $P$ and $H$, we have $Q E=E P=0$ and $E H(I-Q)=I-Q$. Thus, from the above limit equation we have

$$
\begin{aligned}
E x & =E P x+E H(I-Q) N x+Q N x \\
& =E P x+(I-Q) N x+Q N x=N x .
\end{aligned}
$$

We summarize now the hypotheses and the conclusions concerning the operator equation $E x$ $=N x$. We have obtained:

(5.i) Theorem. Let $D E: D(E) \rightarrow Y, D(E) \subset X \subset \mathscr{X}$, E a linear operator, $N: X \rightarrow Y$ a nonnecessarily linear operator, $X, Y$ real Hilbert spaces, $\mathscr{X}$, $\mathscr{Y}$ real Banach or Hilbert spaces with compact injections $j: X \rightarrow \mathscr{X}, j^{\prime}: Y \rightarrow \mathcal{Y}$, with projection operators $P: X \rightarrow X, Q: Y \rightarrow Y$, and decompositions $X=X_{0}+X_{1}, Y=Y_{0}+Y_{1}, X_{0}=P X=\operatorname{ker} E, Y_{1}=(I-Q) Y=$ range $E, X_{0}$ infinitely dimensional, and bounded partial inverse $H: Y_{1} \rightarrow X_{1}$. Let $L=\|H\|$, let $N: X \rightarrow Y$ be a continuous operator, and let $P, Q, H, E, N$ satisfy (a), (b), (c) of Section 2. Let $X_{0 n}, X_{1 n}, Y_{0 n}$ be finite dimensional subspaces of $X_{0}, X_{1}, Y_{0}$ with orthogonal projection operators $R_{n}: X_{1} \rightarrow X_{1}, S_{n}: X_{0} \rightarrow X_{0}, S_{n}^{\prime}: Y_{0} \rightarrow Y_{0}$ with $R_{n} X_{1}=X_{1 n}, S_{n} X_{0}=X_{0 n}, S_{n}^{\prime} Y_{0}=Y_{0 n}$, satisfying (c) of the present section with $\operatorname{dim} X_{0 n}=\operatorname{dim} Y_{0 n}$. Let $\alpha_{n}: Y_{n} \rightarrow X_{n}$ be the operator defined in Section 4.

If there are constants $R_{0}, r>0$ such that (a) for all $x^{*} \in X_{0}, x_{1} \in X_{1},\|x *\| \leqslant R_{0},\left\|x_{1}\right\| \leqslant r$, we have $\left\|N\left(x^{*}+x_{1}\right)\right\| \leqslant L^{-1} r$; and (b) for all $\left\|x^{*}\right\|=R_{0},\left\|x_{1}\right\| \leqslant r$ we have $\left(\alpha_{n} S_{n}^{\prime} Q N\left(x^{*}+x_{1}\right), x^{*}\right) \geqslant 0$ [or $\left.\leqslant 0\right]$, then equation $E x=N x$ has at least one solution $\|x\| \leqslant\left(R_{0}^{2}+r^{2}\right)^{1 / 2}$.

In this theorem (5.i) no requirement is made concerning the behavior of $N\left(x^{*}+x_{1}\right)$ outside 
the set $S=\left[\left(x^{*}, x\right) \in X,\left\|x^{*}\right\| \leqslant R_{0},\left\|x_{1}\right\| \leqslant r\right]$, and thus it allows for an arbitrary growth for $N(x)$ as $\|x\| \rightarrow \infty$.

However, it is easy to see that, if (a) $\|N x\| \leqslant J_{0}$ for some constant $J_{0}$ and all $x \in X$; and (b) for some $R_{0}$ the inequality (b) in (5.i) holds for all $\left\|x^{*}\right\| \geqslant R_{0}$ and $\left\|x_{1}\right\| \leqslant L J_{0}$, then (a), (b) certainly hold for $R_{0}$ as stated in (b) and $r=K=L J_{0}$, where $K$ is the constant of Corollary 1 of Section 3. We have seen in Section 3 that an analogous determination of $R_{0}$ and $r$ can be made in cases of slow growth $\|N x\| \leqslant J_{0}+J_{1}\|x\|^{k}, 0<k<1$, and even in the case that $\|N x\| \leqslant J_{0}+J_{1}\|x\|^{k}$ for some $k \geqslant 1$ provided $J_{1}$ is sufficiently small (cf. [5,6] for cases of arbitrary growth).

Remark 1. Note that the modified bifurcation equation (5.2), or $\alpha_{n} S_{n}^{\prime} Q N x=0$, can always be replaced by the equation

$$
J_{n} \alpha_{n} S_{n}^{\prime} Q N x=0
$$

where $J_{n}: X_{0 n} \rightarrow X_{0 n}$ is an invertible operator. When this is done, we shall require that (b) holds with the inequality replaced by

$$
\left(J_{n} \alpha_{n} S_{n}^{\prime} Q N x, x^{*}\right) \geqslant 0 \quad[\text { or } \leqslant 0] .
$$

The following corollary of $(5 . i)$ is of interest. Again $L=\|H\|$.

(5.ii) Let $N: X \rightarrow Y$ be a continuous map, and let there be monotone nondecreasing nonnegative functions $\alpha(R), \beta(R), R>0$, such that:

(i) $x \in X$, $\|x\| \leqslant R$ implies $\|N x\| \leqslant \alpha(R)$;

(ii) $x_{1}, x_{2} \in X,\left\|x_{1}\right\|,\left\|x_{2}\right\| \leqslant R$ implies $\left\|N x_{1}-N x_{2}\right\| \leqslant \beta(R)\left\|x_{1}-x_{2}\right\|$. Let us assume further that

(iii) there are numbers $R_{0}, r>0$ such that $L \beta\left(\left(R_{0}^{2}+r^{2}\right)^{1 / 2}\right)<1, L \alpha\left(\left(R^{2}+r^{2}\right)^{1 / 2}\right) \leqslant r$; and

(iv) $\left(\alpha_{n} S_{n}^{\prime} Q N\left(x^{*}+x_{1}\right), x^{*}\right) \geqslant 0$ [or $\left.\leqslant 0\right]$ for all $\left\|x^{*}\right\|=R_{0}$ and $\left\|x_{1}\right\| \leqslant r$.

Then the equation $E x=N x$ has at least one solution $x=x^{*}+x_{1},\left\|x^{*}\right\| \leqslant R_{0},\left\|x_{1}\right\| \leqslant r$.

Proof. We proceed as for (5.i) where now we first follow ([12], no. 5). Let $B$ denote the set of all $x=x^{*}+x_{1}, x^{*} \in B_{0}=\left[x^{*} \in X_{0},\left\|x^{*}\right\| \leqslant R_{0}\right], x_{1} \in B_{1}=\left[x_{1} \in X_{1},\left\|x_{1}\right\| \leqslant r\right]$. For every $n$, let $B_{n}$ denote the set of all $x=x^{*}+X_{1}, x^{*} \in B_{0 n}=S_{n} P B, x_{1} \in B_{1 n}=R_{n}(I-P) B$. Then, the truncated auxiliary equation $x=S_{n} P x+R_{n} H(I-Q) N x$, for each arbitrary but fixed $x_{n}^{*} \in$ $\in B_{0 n}=S_{n} P B$, becomes $x_{1}=R_{n} H(I-Q) N\left(x_{n}^{*}+x_{1}\right), x_{1} \in B_{1 n}$, whose second member is a contraction map of $B_{1 n}$ into itself. Hence, the same auxiliary equation has a unique solution $x_{n 1}=\tau\left(x_{n}^{*}\right) \in B_{1 n}$, or $x=x_{n}^{*}+\tau\left(x_{n}^{*}\right)=T\left(x_{n}^{*}\right)$, where now $T$ is a continuous map from $B_{0 n}$ into $B_{0 n} \times B_{1 n}$. The truncated bifurcation equation is now reduced to $\alpha_{n} S_{n}^{\prime} Q N T\left(x_{n}^{*}\right)=0, x_{n}^{*} \in$ $B_{0 n}$, and the inequality in (iv) can be used to obtain the existence of a solution $x_{0 n}^{*}$ of this equation. Then, system $(5.1),(5.2)$ has a solution $x_{n}=x_{0 n}^{*}+\tau\left(x_{0 n}^{*}\right)$. Since $\left\|x_{0 n}^{*}\right\| \leqslant R_{0}\left\|x_{n}\right\|=$ $\left\|T\left(x_{0 n}^{*}\right)\right\| \leqslant\left(R_{0}^{2}+r^{2}\right)^{1 / 2}=R$, and these bounds are independent of $n$, we can proceed as for (5.i) to obtain the existence of a solution $x \in B=B_{0} \times B_{1}$ of the equation $E x=N x, x=$ $x^{*}+x_{1},\left\|x^{*}\right\| \leqslant R_{0},\left\|x_{1}\right\| \leqslant r$. 
6. THE SPACES $A_{p m}$

We discuss here in detail the class $A_{p m}$ of periodic functions. For the sake of completeness we prefer to present here the boundary value problem which has motivated the study of this class. Indeed, we consider the problem of existence of solutions $u(t, x)$, periodic in $t$ of period $2 \pi$, for the differential equation and boundary conditions.

$$
\begin{gathered}
D_{t}^{2} u+(-1)^{p} D_{x}^{2 p}=f(t, x, u, \ldots), \quad 0<x<\pi, \quad-\infty<t<+\infty, \\
u(t, 0)=u(t, \pi)=0, \quad-\infty<t<+\infty, \\
D_{x}^{2 s} u(t, 0)=D_{x}^{2 s} u(t, \pi)=0, \quad s=1,2, \ldots, p-1, \\
u(t+2 \pi, x)=u(t, x), \quad 0<x<\pi, \quad-\infty<t<-\infty .
\end{gathered}
$$

Thus, for $p=1$, we have the wave problem $u_{t t}-u_{x x}=0$ with the condition $u(t, 0)=$ $u(t, \pi)=0$ and $2 \pi$-periodicity in $t$.

Let $G=[0,2 \pi] \times[0, \pi]$. Let $D$ denote the set of all real valued functions $u(t, x), 2 \pi$ periodic in $t$, of class $C^{\infty}$ in $G$, and such that $D_{x}^{2 k} u(t, 0)=D_{x}^{2 k} u(t, \pi)=0, k=0,1, \ldots$ Let $A_{p m}$ denote the completion of $D$ under the norm

$$
\|u\|_{p m}=\left(\iint_{G}\left(\left(D_{t}^{m} u\right)^{2}+\left(D_{x}^{p m} u\right)^{2}\right) \mathrm{d} t \mathrm{~d} x\right)^{1 / 2} .
$$

Then, $A_{p m}$ is a real Hilbert space with inner product

$$
(u, v)_{p m}=\left(D_{t}^{m} u, D_{t}^{m} v\right)+\left(D_{x}^{p m} u, D_{x}^{p m} v\right), \quad u, v \in A_{p m}
$$

where in the second member the inner products are in $L_{2}(G)$.

Let $E$ denote the operator defined by $E u=D_{l}^{2} u+(-1)^{p} D_{x}^{2} u$. Thus, for $m=p=1, E u$ $=u_{t}-u_{x x},(u, v)_{1,1}=\left(u_{t}, v_{t}\right)+\left(u_{x}, v_{x}\right)$. For any $g(t, x) \in A_{p m}$, we may consider the linear problem $E u=g$. We say that $u$ is a weak solution of this problem with boundary conditions (6.2-6.4) provided $u \in A_{p m}$ and $(u, E y)_{L_{2}}=(g, y)_{L_{2}}$ for all $y \in D$. Then, both equation $E u$ $=g$ and boundary conditions $(6.2-6.4)$ are understood in the weak sense. A complete orthonormal system in $A_{p 0}=L_{2}(G)$ is

$$
\left\{e_{k l}\right\}=\left\{2^{1 / 2} \pi^{-1} \sin k t \sin l x, 2^{1 / 2} \pi^{-1} \cos k t \sin l x, \pi^{-1} \sin l x\right\}
$$

whose elements are naturally indexed by $l=1,2, \ldots, k=0, \pm 1, \pm 2, \ldots$ For every element $u \in A_{p m}, u$ has a Fourier development $u=\Sigma_{k l} a_{k l} e_{k l}$, where $\Sigma_{k i}$ ranges over all $l=$ $1,2, \ldots, k=0, \pm 1, \pm 2, \ldots$. The $L_{2}$-integrable functions $D_{t}^{m} u, D_{x}^{p m} u$ have Fourier series which can be obtained by formal differentiation, and thus

$$
\sum_{k l} a_{k l}^{2}\left(k^{2 m}+l^{2 p m}\right)=\|u\|_{p, m}^{2}<+\infty .
$$

(6.i) If $u \in A_{p m}$ then $D_{t}^{\alpha} D_{x}^{\beta} u$ is continuous if $m>\alpha+p^{-1} \beta+(2 p)^{-1}(p+1)$ and then $\left\|D_{t}^{\alpha} D_{x}^{\beta} u\right\|_{\infty} \leqslant c\|u\|_{p m}$, where the constant $c$ depends only on $\alpha, \beta, m, p$. Moreover, for given $\alpha, \beta, p, m, C$, with $m>\alpha+p^{-1} \beta+(2 p)^{-1}(p+1)$, and $C>0$, the functions $u$ with $u \in A_{p m}$, $\|u\|_{p m} \leqslant C$ are uniformly continuous. 
Proof. If $u \in A_{p m}$ then $u(t, x)=\sum_{k l} a_{k l} e_{k l}$ with $\sum_{k l} a_{k l}^{2}\left(k^{2 m}+l^{2 p m}\right)=\|u\|_{p m}^{2}<+\infty$, or $a_{k l}\left(k^{2 m}+p^{p m}\right)^{1 / 2}=b_{k l}$ and $\Sigma b_{k l}^{2}<+\infty$. Note that $\left(k^{2}+l^{2 p}\right)^{m} \leqslant 2^{m-1}\left(k^{2 m}+l^{2 p m}\right)$. From distribution theory then

$$
\begin{aligned}
\left|D_{l}^{\alpha} D_{x}^{\beta} u(t, x)\right| & =\left|\sum_{k l} b_{k l} \frac{D_{l}^{\alpha} D_{x}^{\beta} e_{k l}}{\left(k^{2 m}+l^{2 p m}\right)^{1 / 2}}\right| \\
& \leqslant\left(\sum_{k l} b_{k l}^{2}\right)^{1 / 2}\left[\sum_{k l}\left(\frac{D_{l}^{\alpha} D_{x}^{\beta} e_{k l}}{\left(k^{2 m}+l^{2 p m}\right)^{1 / 2}}\right)^{2}\right]^{1 / 2} \leqslant 2^{(m-1) / 2}\|u\|_{p m}\left(\sum_{k l} \frac{k^{2 \alpha} l^{2 \beta}}{\left(k^{2}+l^{2 p}\right)^{m}}\right)^{1 / 2} .
\end{aligned}
$$

The sum in the series in parenthesis is, up to a multiplicative constant, less than the value of the following double integral, on which we perform the elementary substitution $y=z^{1 / p}$, $y \geqslant 1, z \geqslant 1$, and then we use polar coordinates $x=\rho \cos \theta, z=\rho \sin \theta$,

$$
\begin{aligned}
I & =\int_{1}^{\infty} \int_{1}^{\infty} \frac{x^{2 \alpha} y^{2 \beta}}{\left(x^{2}+y^{2 p}\right)^{m}} \mathrm{~d} x \mathrm{~d} y=\int_{1}^{\infty} \int_{1}^{\infty} \frac{x^{2 \alpha} z^{2 \beta / p}}{\left(x^{2}+z^{2}\right)^{m}}(1 / p) z^{(1 / p)-1} \mathrm{~d} x \mathrm{~d} z \\
& =(1 / p) \int_{0}^{\pi / 2}(\cos \theta)^{2 \alpha}(\sin \theta)^{(2 \beta+1-p) / p} \mathrm{~d} \theta \int_{1}^{\infty} \rho^{2 \alpha+(2 \beta / p)+(1 / p)-2 m} \mathrm{~d} \rho .
\end{aligned}
$$

This integral has a finite value for $2 \alpha+(2 \beta / p)+(1 / p)-2 m<-1$ or $m>\alpha+p^{-1} \beta+$ $(2 p)^{-1}(1+p)$. Thus for $p=2$, we obtain the requirement $m>\alpha+2^{-2} \beta+3 / 4$, which is certainly satisfied if $m \geqslant \alpha+2^{-1} \beta+1$. The same series above

$$
\sum_{k l} b_{k l}\left(k^{2 m}+l^{p m}\right)^{-1 / 2} D_{t}^{\alpha} D_{x}^{\beta} e_{k l}
$$

converges uniformly. To prove this we have only to show that it is uniformly Cauchy. Indeed, any partial sum $\Sigma^{\prime}$ with indices, say $M \leqslant|k|+l \leqslant P$, is in absolute value

$$
\begin{aligned}
& \leqslant\left(\Sigma^{\prime} b_{k l}^{2}\right)^{1 / 2}\left(\Sigma^{\prime}\left(\left(k^{2 m}+l^{p m}\right)^{-1 / 2} D_{l}^{\alpha} D_{x}^{\beta} e_{k l}\right)^{2}\right)^{1 / 2} \\
& \leqslant\|u\|_{p m}\left(\Sigma^{\prime}\left(\left(k^{2 m}+l^{p m}\right)^{-1 / 2} D_{t}^{\alpha} D_{x}^{\beta} e_{k l}\right)^{2}\right)^{1 / 2},
\end{aligned}
$$

where the first factor in the last term in bounded, and the second factor approaches zero as $M, P \rightarrow+\infty$, independently of $t, x$ and $u$. This proves (6.i).

(6.ii) If $u \in A_{p m}$ then $D_{t}^{\alpha} D_{x}^{\beta} u \in L_{q}$ provided $\alpha+p^{-1} \beta+(2 p)^{-1}(p+1) \geqslant m$ and $2<q<$ $(2 p+2) /(p+1+2 \alpha p+2 \beta-2 m p)$. Then $\left\|D_{t} D_{x} u\right\|_{L_{q}} \leqslant c\|u\|_{p m}$ where the constant $c$ depends only on $\alpha, \beta, m, p, q$.

Proof. As before

$$
D_{l}^{\alpha} D_{x}^{\beta} u(t, x)=\sum_{k l} b_{k l}\left(k^{2 m}+l^{2 p m}\right)^{-1 / 2} D_{t}^{\alpha} D_{x}^{\beta} e_{k l} .
$$

Let us find a number $\zeta, 1<\zeta<2$, such that

$$
S=\sum_{k l}\left|b_{k l}\left(k^{2 m}+l^{2 p m}\right)^{-1 / 2} k^{\alpha} l^{\beta}\right|^{\zeta}<+\infty
$$

This series can be majorized by

$$
\left(\sum_{k l}\left(\left|b_{k l}\right|^{\zeta}\right)^{2 / \zeta}\right)^{\zeta / 2}\left(\sum_{k l}\left(\left(\left(k^{2}+l^{2 p}\right)^{-m / 2} k^{\alpha} l^{\beta}\right)^{\zeta}\right)^{2 /(2-\xi)}\right)^{1-\zeta / 2} .
$$


Thus, it is enough to prove that the series inside the last parenthesis is convergent. For this, it is enough to show the convergence of the following double integral, on which we perform as before the substitution $y=z^{1 / p}, y \geqslant 1, z \geqslant 1$, and the change into polar coordinates $x=$ $\rho \cos \theta, z=\rho \sin \theta$ :

$$
\begin{aligned}
& \int_{1}^{\infty} \int_{1}^{\infty}\left(x^{\alpha} y^{\beta}\left(x^{2}+y^{2 p}\right)^{-m / 2}\right)^{2 \xi(2-\zeta)} \mathrm{d} x \mathrm{~d} y \\
& \quad=\int_{1}^{\infty} \int_{1}^{\infty}\left(x^{\alpha} z^{\beta / p}\left(x^{2}+z^{2}\right)^{-m / 2}\right)^{2 \xi /(2-\xi)}(1 / p) z^{(1 / p)-1} \mathrm{~d} x \mathrm{~d} z \\
& \quad=(1 / p) \int_{0}^{\pi / 2}(\cos \theta)^{2 \alpha \zeta(2-\xi)}(\sin \theta)^{2 \beta \xi(2-\xi) p+(1-p) / p} \mathrm{~d} \theta \cdot \int_{1}^{\infty} \rho^{(\alpha+\beta / p-m)(2 \zeta /(2-\xi))+1 / p} \mathrm{~d} \rho .
\end{aligned}
$$

This integral has a finite value for $(\alpha+\beta / p-m)(2 \xi /(2-\xi))+1 / p<-1$, or $\zeta>\zeta_{0}=$ $(2 p+2)(2 m p+p+1-2 \alpha p-2 \beta)^{-1}$, provided $2 m p+p+1-2 \alpha p-2 \beta>0$. This condition is satisfied and we have $1 \leqslant \zeta_{0}<2$ if $2 m p-p-1 \leqslant 2 \alpha p+2 \beta<2 m p$. Now, for any such $\zeta$, series (6.5) is convergent, and by the Young-Hausdorff theorem (cf. [18, Vol. 2, p. 600$]), D_{t}^{\alpha} D_{x}^{\beta} u$ is $L_{q}$ integrable for $q=\zeta(\zeta-1)^{-1}$, that is, $D_{t}^{\alpha} D_{x}^{\beta} u \in L_{q}$ for all $q<\zeta_{0}\left(\zeta_{0}\right.$ $-1)^{-1}$, or

$$
q<(2 p+2)(p+1+2 a p+2 \beta-2 m p)^{-1},
$$

and $\left\|D_{t}^{\alpha} D_{x}^{\beta} u\right\|_{q} \leqslant S$. Under the assumptions of (6.ii) the inequalities above are all satisfied.

(6.iii) If $u \in A_{p m}$, then $D_{t}^{\alpha} D_{x}^{\beta} u \in L_{2}$ if $\alpha+p^{-1} \beta \leqslant m$, and then $\left\|D_{t}^{\alpha} D_{x}^{\beta} u\right\|_{L_{2}} \leqslant c\|u\|_{m p}$ where $c$ is a constant depending only on $\alpha, \beta, p, m$.

Proof. As before we have

$$
D_{i}^{\alpha} D_{x}^{\beta} u(t, x)=\sum_{k l} b_{k}\left(k^{2 m}+l^{2 p m}\right)^{-1 / 2} D_{l}^{\alpha} D_{x}^{\beta} e_{k l}
$$

where $\|u\|_{m p}^{2}=\Sigma_{k l} b_{k l}^{2}<+\infty$. We have to prove that

$$
\iint_{G}\left(D^{\alpha} D^{\beta} u(t, x)\right)^{2} \mathrm{~d} t \mathrm{~d} x=\sum_{k l} b_{k}^{2}\left(k^{2 m}+l^{2 p m}\right)^{-1} k^{2 \alpha} q^{2 \beta}<+\infty .
$$

Indeed, for all real numbers $A, b \geqslant 0$ and integers $m \geqslant 1$, we have $A^{m}+B^{m} \leqslant(A+B)^{m}$ $\leqslant 2^{m-1}\left(A^{m}+B^{m}\right)$. Hence, for $\alpha+p^{-1} \beta \leqslant m$, we also have

$$
\begin{aligned}
k^{2 \alpha} p^{\beta} & =k^{2 \alpha} l^{2 p(\beta / p)} \leqslant\left(k^{2}+l^{2 p}\right)^{\alpha}\left(k^{2}+l^{2 p}\right)^{(\beta / p)} \\
& \leqslant\left(k^{2}+l^{2 p}\right)^{\alpha+\beta l p} \leqslant\left(k^{2}+l^{2 p}\right)^{m} \leqslant 2^{m-1}\left(k^{2 m}+l^{2 p m}\right) .
\end{aligned}
$$

In other words, the last series is majorized by $2^{m-1} \Sigma_{k l} b_{k l}^{2}$.

As an immediate application of the above statements we note the following:

For $p=2, m=0$, then $A_{20}=L_{2}$.

For $p=2, m=1,\|u\|_{A_{21}}^{2}=\left\|u_{t}\right\|_{L_{2}}^{2}+\left\|u_{x x}\right\|_{L_{2}}^{2}$, and for $u \in A_{21}$, then $u \in C, u_{x} \in L_{q}$ for any $q<6, u_{t}, u_{x x} \in L_{2}$, and there are constants $\mu_{0}, \mu_{1}, \mu_{0}^{\prime},\left(\mu_{1}\right.$ depending on $q$ ) such that $\|u\|_{\infty} \leqslant$ $\mu_{0}\|u\|_{A_{21}},\left\|u_{x}\right\|_{L_{q}} \leqslant \mu_{1}\|u\|_{A_{21}}$ for any $q<6,\left\|u_{\|}\right\|_{L_{2}} \leqslant\|u\|_{A_{21}},\left\|u_{x x}\right\| \leqslant\|u\|_{A_{21}},\|u\|_{L_{2}} \leqslant \mu_{u}^{\prime} \mid\|u\|_{A_{21}}$ (with $\left.\mu_{0}^{\prime} \leqslant(\text { meas } G)^{1 / 2} \mu_{0}\right)$. 
For $p=2, m=2,\|u\|_{A_{22}}^{2}=\left\|u_{t 1}\right\|_{L_{2}}^{2}+\left\|u_{x x x x}\right\|_{L_{2}}^{2}$, and for $u \in A_{22}$, then $u, u_{t}, u_{x}, u_{x x} \in C, u_{t x}, u_{x x x}$ $\in L_{q}$ for any $q<6$, and $u_{t}, u_{x x x} \in L_{q}$ for any $q<6$, and $u_{t}, u_{t x x}, u_{x x x x} \in L_{2}$. Thus, $u_{x}, u_{t}$, $u_{x}, u_{x x} \in A_{21}$ also. Moreover, there are constants as above such that $\|u\|_{\infty} \leqslant \mu_{0}\|u\|_{A_{22}},\left\|u_{t}\right\|_{\infty}$, $\left\|u_{x}\right\|_{\infty},\left\|u_{x x}\right\| \leqslant \mu_{1}\|u\|_{A_{22}},\left\|u_{t x}\right\|_{L_{q}},\left\|u_{x x x}\right\|_{L_{q}} \leqslant \mu_{2}\|u\|_{A_{22}}, 2 \leqslant q<6,\left\|u_{t t}\right\|_{L_{2}},\left\|u_{x x x}\right\|_{L_{2}},\left\|u_{x x x x}\right\|_{L_{2}} \leqslant$ $\mu_{3}\|u\|_{A_{22}}$

For $p=2, m=3,\|u\|_{A_{23}}^{2}=\left\|u_{t t t}\right\|_{L_{2}}^{2}+\left\|u_{x x x x x x}\right\|_{L_{2}}^{2}$, and for $u \in A_{23}$, then $u, u_{t}, u_{x}, u_{t i}, u_{x x}, u_{t x x}$, $u_{x x x}, u_{x x x x} \in C, u_{t x}, u_{x x x x x} \in L_{q}$ for all $q<6$, and $u_{t t t}, u_{t t x x}, u_{x x x x x x} \in L_{2}$, and there are constants as above relating the norms $L_{\alpha}, L_{q}, L_{2}$ to the norm in $A_{23}$.

For $p=1, m=1,\|u\|_{A_{11}}^{2}=\left\|u_{t}\right\|_{L_{2}}^{2}+\left\|u_{x}\right\|_{L_{2}}^{2}$, and for $u \in A_{11}$, then $u \in L_{q}$ for any $q, 1 \leqslant$ $q<\infty, u_{t}, u_{x} \in L_{2}$, and $\|u\|_{L_{q}} \leqslant \mu_{1 q}\|u\|_{A_{11}},\left\|u_{t}\right\|_{L_{2}} \leqslant\|u\|_{A_{11}},\left\|u_{x}\right\|_{L_{2}} \leqslant\|u\|_{A_{11}}$ for a suitable constant $\mu_{1 q}>0$.

For $p=1, m=2,\|u\|_{A_{12}}^{2}=\left\|u_{t t}\right\|_{L_{2}}^{2}+\left\|u_{x x}\right\|_{L_{2}}^{2}$, and for $u \in A_{12}$, then $u \in C, u_{t}, u_{x} \in L_{q}$ for any $q, \quad 1 \leqslant q<\infty, \quad u_{t t} \in L_{2}, \quad u_{x x} \in L_{2}, \quad u_{t x} \in L_{2}, \quad$ and $\quad\|u\|_{\infty} \leqslant \mu_{0}\|u\|_{A_{11}}, \quad\left\|u_{t}\right\|_{L_{q}} \leqslant \mu_{1 q}\|u\|_{A_{11}}$. $\left\|u_{t x}\right\| \leqslant \mu_{1}\|u\|_{A_{11}},\left\|u_{t t}\right\|_{L_{2}} \leqslant\|u\|_{A_{11}},\left\|u_{x x}\right\| \leqslant\|u\|_{A_{11}}$ for suitable constants $\mu_{1}, \mu_{1 q}, \mu_{1}>0$.

Remark 2. The imbedding theorems proved above have corresponding compact imbedding statements. We do not develop this point here. However, the following case will be needed below. If $u \in A_{22}$, then $u, u_{t}, u_{x} \in C$, and $u_{t t} \in L_{2}, u_{t x} \in L_{q}$ for any $2 \leqslant q \leqslant 6, u_{x x} \in C$, as stated in the third example above. In particular $u_{t t}, u_{t x}, u_{x x} \in L_{2}$, and

$$
\|u\|_{\infty},\left\|u_{t}\right\|_{\infty},\left\|u_{x}\right\|_{\infty},\left\|u_{t a}\right\|_{L_{2}},\left\|u_{t}\right\|_{L_{2}},\left\|u_{x x}\right\|_{L_{2}} \leqslant \gamma\|u\|_{A_{22}}
$$

for some absolute constant $\gamma$. Thus, $u_{t}, u_{x}$ both belong to the Sobolev space $W^{1.2}(G), G$ a two dimensional interval. By the Rellich-Kondrashov theorem (cf. [1, (6.2), Part I, p. 144]) the embedding $W^{1,2}(G) \rightarrow W^{0, q}(G)$ is compact for every $1 \leqslant q<\infty$. In other words, if $\left[u_{s}, s=\right.$ $1,2, \ldots]$ is a sequence of elements $u_{s} \in A_{22}$ with $\left\|u_{s}\right\|_{A_{22}} \leqslant M$ for all $s$, then the functions $u_{s}$ are equi-Lipschitzian, and for a suitable subsequence, say still [s], then $u_{s} \rightarrow u$ uniformly to a Lipschitz function $u$, and $\left(u_{s}\right)_{t} \rightarrow u_{t},\left(u_{s}\right)_{x} \rightarrow u_{x}$ strongly in $L_{q}(G)$ for any $1 \leqslant q<\infty$, and even pointwise almost everywhere.

Remark 3. Some of the above results can be seen also in $[16,17,20]$. Our proofs are not always the same, but we cover this matcrial for the sake of completeness.

\section{SERIES SOLUTIONS FOR THE WAVE EQUATION}

In connection with the previous considerations, the following precise estimates will be needed in Section 14.

Let $\left[e_{k l}, k, l=0, \pm 1, \pm 2, \ldots\right]$ denote the system generated by $\exp (i k t) \exp (i l \tau)$ in $\mathbb{R}^{2}$, and orthogonal in $G=[0,2 \pi] \times[0,2 \pi]$. Let $u(t, \tau)=\Sigma_{k^{2} \neq p} b_{k l} e_{k l}$ be any function in $A_{11}, 2 \pi-$ periodic in $t$ and $\tau$, thus $u, u_{t}, u_{\tau}, \in L_{2}(G)$, and

$$
K^{2}=\sum_{k^{2} \neq l} b_{k l}^{2}\left(k^{2}+l^{2}\right)=\left\|u_{t}\right\|_{L_{2}}^{2}+\left\|u_{u}\right\|_{L_{2}}^{2}<+\infty .
$$

We shall consider the function

$$
v(t, \tau)=\sum_{k^{2} \neq l^{2}} b_{k}\left(-k^{2}+l^{2}\right)^{-1} e_{k l},
$$


and for every $\Lambda>1$, also the function

$$
w(t, \tau)=\sum_{\left|-k^{2}+R\right| \geqslant \Lambda} b_{k l}\left(-k^{2}+l^{2}\right)^{-1} e_{k l}
$$

(7.i) For $u$ as above, $v$ and $w$ have bounded first order partial derivatives and

$$
\begin{aligned}
\left|v_{t}(t, \tau)\right|,\left|v_{\tau}(t, \tau)\right| & \leqslant(\pi / \sqrt{6}) K=1.2826 K, \\
\left|w_{t}(t, \tau)\right|,\left|w_{\tau}(t, \tau)\right| & \leqslant 2 K\left(\Lambda^{1 / 2}-1\right)^{-1 / 2}, \\
|v(t, \tau)| & \leqslant 3.06126 K .
\end{aligned}
$$

Proof. Let $c_{k l}=b_{k l}\left(k^{2}+l^{2}\right)^{1 / 2}$, so that $\Sigma c_{k l}^{2}=K^{2}<+\infty$, where $\Sigma$ denotes a sum ranging over all $k, l=0, \pm 1, \pm 2, \ldots, k^{2} \neq l^{2}$. Then

$$
\begin{aligned}
& v=\Sigma b_{k l}\left(-k^{2}+l^{2}\right)^{-1} e_{k l}=\Sigma c_{k}\left(k^{2}+l^{2}\right)^{-1 / 2}\left(-k^{2}+l^{2}\right)^{-1} e_{k l} \\
& v_{t}=\Sigma c_{k l} k\left(k^{2}+l^{2}\right)^{-1 / 2}\left(-k^{2}+l^{2}\right)^{-1} e_{k l}^{\prime}
\end{aligned}
$$

where $e_{k l}^{\prime}$ is derived from $e_{k l}$ by replacing $\sin k t, \cos k t$ by $\cos k t,-\sin k t$. Then, since $\left|e_{k l}\right| \leqslant$ $\pi^{-1}$, we have

$$
\begin{aligned}
\left|v_{l}(t, \tau)\right| & \leqslant\left(\Sigma c_{l}^{2}\right)^{1 / 2}\left(\Sigma k^{2}\left(k^{2}+l^{2}\right)^{-1}\left(-k^{2}+l^{2}\right)^{-2} \pi^{-2}\right)^{1 / 2} \\
& \leqslant \pi^{-1} K\left(4 \sum_{k, l \geqslant 0, k \neq l} k^{2}\left(k^{2}+l^{2}\right)^{-1}(k+l)^{-2}(k-l)^{-2}\right)^{1 / 2} \\
& \leqslant \pi^{-1} K\left(4 \sum_{k, l \geqslant 0, k \neq l}(k+l)^{-2}(k-l)^{-2}\right)^{1 / 2} \\
& \leqslant \pi^{-1} K\left(4 \sum_{s=1}^{\infty} s^{-2} \Sigma^{\prime}(2 k-s)^{-2}\right)^{1 / 2}
\end{aligned}
$$

where the inner sum is extended to all $k=0,1, \ldots, s$ with $2 k \neq s$. Thus, the inner sum is $\leqslant 2\left(1+3^{-2}+5^{-2}+\ldots\right) \leqslant 2\left(\pi^{2} / 8\right)$ if $s$ is odd, and $\leqslant 2\left(2^{-2}+4^{-2}+\ldots\right)$ if $s$ is even. Since $1+2^{-2}+3^{-2}+\ldots=\pi^{2} / 6$, we have in any case

$$
\begin{aligned}
\left|v_{l}(t, \tau)\right| & \leqslant \pi^{-1} K(\pi / 2)\left(\sum_{1}^{\infty} s^{-2}\right)^{1 / 2} \\
& \leqslant \pi^{-1} K(\pi / 2) 2(\pi / \sqrt{6})=(\pi / \sqrt{6}) K=1 \cdot 2826 K,
\end{aligned}
$$

and analogously for $v_{\tau}$. The computations for $w$ are the same, where the sum with respect to $s$ ranges over all integers $\geqslant \Lambda^{1 / 2}$, and

Analogously, we have

$$
\sum_{s \geqslant \Lambda^{1 / 2}} s^{-2} \leqslant \int_{\Lambda^{1 / 2}-1}^{+\infty} s^{-2} \mathrm{~d} s=\left(\Lambda^{1 / 2}-1\right)^{-1}
$$

$$
\begin{aligned}
|v(t, \tau)| & \leqslant\left(\Sigma c_{k l}^{2}\right)^{1 / 2}\left(\Sigma\left(k^{2}+l^{2}\right)^{-1}\left(-k^{2}+l^{2}\right)^{-2} \pi^{-2}\right)^{1 / 2} \\
& \leqslant \pi^{-1} K\left(4 \sum_{k, l \geqslant 0, k \neq l}\left(k^{2}+l^{2}\right)^{-1}(k+l)^{-2}\right)^{1 / 2} \\
& \leqslant \sqrt{2} \pi^{-1} K\left(4 \sum_{s=1}^{\infty} s^{-4} \Sigma^{\prime}(2 k-s)^{-2}\right)^{1 / 2}
\end{aligned}
$$


where $1+2^{-4}+3^{-4}+\ldots=1 \cdot 08232$. Thus

with

$$
|v(t, \tau)| \leqslant 2 \sqrt{2} \pi^{-1} K(\pi / 2)(1.08232)=1.53063 K,
$$

$$
K=\left(\Sigma c_{k}^{2}\right)^{1 / 2}=\left(\iint_{G}\left(u_{t}^{2}+u_{t}^{2}\right) \mathrm{d} t \mathrm{~d} \tau\right)^{1 / 2}
$$

Now let $u(t, \tau)=\Sigma b_{k l} e_{k l}$ be any function in $A_{11}$, where now the sum ranges over all $k, l=0$, $\pm 1, \pm 2, \ldots$, and let $U(t, \tau)$ denote the function $U(t, \tau)=\Sigma_{k^{2}=l^{2}} b_{k l} e_{k l}$, where now the sum ranges over all $k, l=0, \pm 1, \pm 2, \ldots$ with $k^{2}=l^{2}$, or $k= \pm l$. Let $K_{0}$ denote the constant $K_{0}^{2}=\Sigma_{k^{2}=l^{2}} b_{k l}^{2}\left(k^{2}+l^{2}\right)$.

(7.ii) For $u \in A_{11}$, then $U$ is a Lipschitz function satisfying

$$
|U(t+h, \tau)-U(t, \tau)| \leqslant \nu_{1} K_{0}|h|, \quad|U(t, \tau+k)-U(t, \tau)| \leqslant \nu_{1} K_{0}|k|, \quad|U(t, \tau)| \leqslant \nu_{1} K_{0}
$$

where $\nu_{1}$ is an absolute constant.

Proof. Using Fourier series we have

$$
\begin{aligned}
u & =\sum_{m, n=0}^{\infty} \lambda_{m n}\left(a_{m n} \cos m t \cos n \tau+b_{m n} \sin m t \cos n \tau+c_{m n} \cos m t \sin n \tau\right. \\
& \left.+d_{m n} \sin m t \sin n \tau\right),
\end{aligned}
$$

where $\lambda_{00}=\frac{1}{4}, \lambda_{m n}=\frac{1}{2}$ if $m=0, n \geqslant 1$ and if $m \geqslant 1, n=0, \lambda_{m n}=1$ otherwise, and we denote by $M$ the constant

$$
M=\left[\sum_{n=1}^{\infty}\left(a_{n n}^{2}+b_{n n}^{2}+c_{n n}^{2}+d_{n n}^{2}\right) n^{2}\right]^{1 / 2}
$$

Then we have

$$
\begin{aligned}
U(t, \tau) & =\left(\frac{1}{4}\right) a_{00}+\sum_{n=1}^{\infty}\left(a_{n n} \cos n t \cos n \tau+b_{n n} \sin n t \cos n \tau+c_{n n} \cos n t \sin n \tau\right. \\
& \left.+d_{n n} \sin n t \sin n \tau\right), \\
|U(t, \tau)| & \leqslant\left(\frac{1}{4}\right)\left|a_{00}\right|+\left[\sum_{n=1}^{\infty}\left(a_{n n}^{2}+b_{n n}^{2}+c_{n n}^{2}+d_{n n}^{2}\right) n^{2}\right]^{1 / 2} \\
& \times\left[\sum_{n=1}^{\infty} n^{-2}\left(\left(\cos ^{2} n t+\sin ^{2} n t\right) \cos ^{2} n \tau+\left(\cos ^{2} n t+\sin ^{2} n t\right) \sin ^{2} n \tau\right)\right]^{1 / 2} \\
& =\left(\frac{1}{4}\right)\left|a_{00}\right|+\left[\sum_{n=1}^{\infty}\left(a_{n n}^{2}+b_{n n}^{2}+c_{n n}^{2}+d_{n n}^{2}\right) n^{2}\right]^{1 / 2}\left[\sum_{n=1}^{\infty} n^{-2}\right]^{1 / 2} \\
& =\left(\frac{1}{4}\right)\left|a_{00}\right|+M\left(\sum_{n=1}^{\infty} n^{-2}\right)^{1 / 2}=\left(\frac{1}{4}\right)\left|a_{00}\right|+M(\pi / \sqrt{6}) .
\end{aligned}
$$


Analogously

$$
\begin{aligned}
U(t+h, \tau)-U(t, \tau) & =\sum_{n=1}^{\infty}\left[a_{n n}(\cos n(t+h) \cos n \tau-\cos n t \cos n \tau)\right. \\
& +b_{n n}(\sin n(t+h) \cos n \tau-\sin n t \cos n \tau) \\
& +c_{n n}(\cos n(t+h) \sin n \tau-\cos n t \sin n \tau) \\
& \left.+d_{n n}(\sin n(t+h) \sin n \tau-\sin n t \sin n \tau)\right] \\
& =(h / 2) \sum_{n=1}^{\infty}\left[a_{n n} \cos n \tau(-2 \sin n(t+h / 2))\right. \\
& +b_{n n} \cos n \tau(2 \cos n(t+h / 2)) \\
& +c_{n n} \sin n \tau(-2 \sin n(t+h / 2)) \\
& \left.+d_{n n} \sin n \tau(2 \cos n(t+h / 2))\right] n \sigma_{n}(h)
\end{aligned}
$$

where $\sigma_{n}(h)=\sin (n h / 2) /(n h / 2)$. Since $\left|\sigma_{n}(h)\right| \leqslant 1$, we have

$$
\begin{aligned}
|U(t+h)-U(t, \tau)| & \leqslant|(h / 2)|\left[\sum_{n=1}^{\infty}\left(a_{n n}^{2}+b_{n n}^{2}+c_{n n}^{2}+d_{n n}^{2}\right) n^{2}\right]^{1 / 2} \\
& \times\left[\sum_{n=1}^{\infty} n^{-2}\left(\cos ^{2} n t+\sin ^{2} n \tau\right)\left(4 \sin ^{2} n(t+h / 2)\right)\right. \\
& \left.+\left(\cos ^{2} n \tau+\sin ^{2} n \tau\right)\left(4 \cos ^{2} n(t+h / 2)\right)\right]^{1 / 2} \\
& \leqslant|h| M\left(\sum_{n=1}^{\infty} n^{-2}\right)^{1 / 2}=(\pi / \sqrt{6}) M|h|,
\end{aligned}
$$

with

$$
\pi / \sqrt{6}=1.28255, \quad M^{2}=\sum_{n=1}^{\infty}\left(a_{n n}^{2}+b_{n n}^{2}+c_{n n}^{2}+d_{n n}^{2}\right) n^{2} \leqslant \pi^{-2} \iint_{G} u_{l}^{2} \mathrm{~d} t \mathrm{~d} t
$$

Also

$$
\begin{aligned}
& |U(t+h, \tau)-U(t, \tau)| \leqslant 6^{-1 / 2}|h|\left\|u_{n}\right\|_{L_{2}}, \\
& \mid U(t, \tau) \leqslant\|u\|_{L_{2}}+6^{-1 / 2}\left\|u_{\|_{2}}\right\|_{L_{2}}
\end{aligned}
$$

with $6^{-1 / 2}=0 \cdot 40825$.

\section{COMPLEMENTARY REMARKS}

In the already quoted work by Petzeltova [20] of the boundary value problem of Section 12 , an unnecessary restriction was made on the data (cf. [20]) which will be eliminated in our analysis in Section 12. To do this the following remarks will be relevant.

First let us note the following elementary solutions to the linear equations below:

(i) $u_{t t}+u_{x x x x}=1$, or equivalently

$$
u_{t t}+u_{x x x x}=(4 / \pi) \sum_{l=1}^{\infty}(2 l-1)^{-1} \sin (2 l-1) x,
$$


with boundary conditions

$$
\begin{aligned}
& u(t, 0)=u(t, \pi)=u_{x x}(t, 0)=u_{x x}(t, \pi)=0, \\
& u(t+2 \pi)=u(t, \pi)
\end{aligned}
$$

has the solution

$$
\begin{aligned}
u(t, x) & =24^{-1} x^{4}-12^{-1} \pi x^{3}+24^{-1} \pi^{3} x \\
& =(4 / \pi) \sum_{l=1}^{\infty}(2 l-1)^{-5} \sin (2 l-1) x
\end{aligned}
$$

(ii) $u_{n}+u_{x x x x}=\sin k t$ for $k^{2} \neq(2 l-1)^{4}$, any $l$, or equivalently

$$
u_{t}+u_{x x x x}=(4 / \pi) \sum_{l=1}^{\infty}(2 l-1)^{-1} \sin k t \sin (2 l-1) x,
$$

with boundary conditions (8.1), has the solution

$$
u(t, x)=(4 / \pi) \sum_{l=1}^{\infty}(2 l-1)^{-1}\left[(2 l-1)^{4}-k^{2}\right]^{-1} \sin k t \sin (2 l-1) x .
$$

Analogous series hold for $\cos k t$ replacing $\sin k t$.

(iii) $u_{n}+u_{x x x x}=x$, or equivalently

$$
u_{t}+u_{x x x x}=2 \sum_{l=1}^{\infty}(-1)^{l+1} l^{-1} \sin l x
$$

with boundary conditions (8.1) has the solution

$$
\begin{aligned}
u(t, x) & =120^{-1} x^{5}-36^{-1} \pi^{2} x^{3}+(360)^{-1} 7 \pi^{4} x \\
& =2 \sum_{l=1}^{\infty}(-1)^{l+1} l^{-5} \sin l x .
\end{aligned}
$$

(iv) $u_{t}+u_{x x x x}=x \sin k t$ for $k^{2} \neq l^{4}$, any $l$, or

$$
u_{n}+u_{x x x x}=2 \sum_{l=1}^{\infty}(-1)^{l+1} l^{-1} \sin k t \sin l x
$$

with boundary conditions ( 8.1 ) has the solution

$$
u(t, x)=2 \sum_{i=1}^{\infty}(-1)^{l+1} l^{-1}\left(-k^{2}+l^{4}\right)^{-1} \sin k t \sin l x .
$$

Analogous series hold for $\cos k t$ replacing $\sin k t$.

Let us consider now a slightly more general situation. Let $f_{1}(t), f_{2}(t)$ be periodic functions of period $2 \pi$ and class $C^{1}$, and let $f(t, x),-\infty<t<+\infty, 0 \leqslant x \leqslant \pi$, be the function linear in $x$ with

or

$$
f(t, 0)=f_{1}(t), f(t, \pi)=f_{2}(t),
$$

$$
f(t, x)=f_{1}(t)+x \pi^{-1}\left(f_{2}(t)-f_{1}(t)\right) \text {. }
$$


If

$$
\begin{gathered}
f_{1}(t)=2^{-1} a_{0}+\sum_{k=1}^{\infty}\left(a_{k} \cos k t+b_{k} \sin k t\right), \\
\pi^{-1}\left(f_{2}(t)-f_{1}(t)\right)=2^{-1} c_{0}+\sum_{k=1}^{\infty}\left(c_{k} \cos k t+d_{k} \sin k t\right),
\end{gathered}
$$

then

$$
\begin{aligned}
f(t, x) & =2^{-1} a_{0}+\sum_{k=1}^{\infty}\left(a_{k} \cos k t+b_{k} \sin k t\right) \\
& +\left[2 \sum_{l=1}^{\infty}(-1)^{l+1} l^{-1} \sin l x\right]\left[2^{-1} c_{0}+\sum_{k=1}^{\infty}\left(c_{k} \cos k t+d_{k} \sin k t\right)\right] .
\end{aligned}
$$

Let $f^{*}(t, x)$ denote the same function $f$ written as a double Fourier series with all terms with $k^{2}=l^{4}$ removed. Then, the equation

$$
u_{t t}+u_{x x x x}=f^{*}(t, x)
$$

with boundary conditions (8.1) has the solution

$$
\begin{aligned}
u(t, x) & =\left(2^{-1} a_{0}\right)(4 / \pi) \sum_{l=1}^{\infty}(2 l-1)^{-5} \sin (2 l-1) x \\
& +(4 / \pi) \sum_{k, l}(2 l-1)^{-1}\left[(2 l-1)^{4}-k^{2}\right]^{-1}\left[a_{k} \cos k t+b_{k} \sin k t\right] \sin (2 l-1) x \\
& +2\left(2^{-1} c_{0}\right) \sum_{l=1}^{\infty}(-1)^{l+1} l^{-5} \sin l x \\
& +2 \sum_{k, l}(-1)^{l+1} l^{-1}\left(l^{4}-k^{2}\right)^{-1}\left[c_{k} \cos k t+d_{k} \sin k t\right] \sin l x,
\end{aligned}
$$

where $\Sigma_{k l}$ ranges over all $k, l=1,2, \ldots, k^{2} \neq(2 l-1)^{4}$ in the second series, and $k^{2} \neq l^{4}$ in the fourth series.

(8.i) For $f_{1}(t), f_{2}(t)$ of class $C^{1}$ and $2 \pi$-periodic, the solution (8.3) of equation (8.2) is of class $A_{22}$.

Proof. To prove this it is enough to show that for the functions $u(t, x)$ defined by the second and fourth series (8.3), both $u_{t t}$ and $u_{x x x x}$ are of class $L^{2}$. For the second series we have

$$
\begin{aligned}
u_{x x x x} & =(4 / \pi) \sum_{k l}(2 l-1)^{3}\left[(2 l-1)^{4}-k^{2}\right]^{-1}\left[a_{k} \cos k t+b_{k} \sin k t\right] \sin (2 l-1) x, \\
u_{t t} & \left.=-(4 / \pi) \sum_{k l}(2 l-1)^{-1} k^{2}[2 l-1)^{4}-k^{2}\right]^{-1}\left[a_{k} \cos k t+b_{k} \sin k t\right] \sin (2 l-1) x,
\end{aligned}
$$

where $\sum_{k=1}^{\infty} k^{2}\left(a_{k}^{2}+b_{k}^{2}\right)<+\infty$. Thus, it is enough to show that

$$
\begin{aligned}
& \sum_{k=1}^{\infty} k^{2} a_{k}^{2}\left[\sum_{(2 l-1)^{4} \neq k^{2}}^{\infty}(2 l-1)^{6}\left[(2 l-1)^{4}-k^{2}\right]^{-2} k^{-2}\right]<+\infty, \\
& \sum_{k=1}^{\infty} k^{2} a_{k}^{2}\left[\sum_{\substack{l=1 \\
l}}^{\infty}(2 l-1)^{-2}\left[(2 l-1)^{4}-k^{2}\right]^{-2} k^{2}\right]<+\infty .
\end{aligned}
$$


We shall actually prove that there is a constant $B>0$ such that, for any $k$, we have

$$
\begin{aligned}
& \sum_{l=1}^{\infty}(2 l-1)^{6}\left[(2 l-1)^{4}-k^{2}\right]^{-2}<B k^{2}, \\
& \sum_{l=1}^{\infty}(2 l-1)^{-2}\left[(2 l-1)^{4}-k^{2}\right]^{-2}<B k^{-2},
\end{aligned}
$$

where the terms with $k^{2}=(2 l-1)^{4}$ are omitted.

It is enough we prove that for some constant $C>0$ we have

$$
\begin{aligned}
& I_{1}=\int_{\sqrt{k+1}}^{\infty} x^{6}\left(x^{4}-k^{2}\right)^{-2} \mathrm{~d} x<C k^{2}, \\
& I_{2}=\int_{1}^{\sqrt{k-1}} x^{6}\left(x^{4}-k^{2}\right)^{-2} \mathrm{~d} x<C k^{2}
\end{aligned}
$$

and

$$
\begin{aligned}
& I_{3}=\int_{\sqrt{k+1}}^{\infty} x^{-2}\left(x^{4}-k^{2}\right)^{-2} \mathrm{~d} x<C k^{-2} \\
& I_{4}=\int_{1}^{\sqrt{k-1}} x^{-2}\left(x^{4}-k^{2}\right)^{-2} \mathrm{~d} x<C k^{-2}
\end{aligned}
$$

Indeed, for $k=b^{2}$, or $b=\sqrt{k}$, we have

$$
\begin{aligned}
\int x^{6}\left(x^{4}-b^{4}\right)^{-2} \mathrm{~d} x & =3(16 b)^{-1} \log \left(|x-b||x+b|^{-1}\right) \\
& +3(8 b)^{-1} \arctan (x / b)-4^{-1} x^{3}\left(x^{4}-b^{4}\right)^{-1}+C
\end{aligned}
$$

and hence

$$
\begin{aligned}
I_{1} & =3 \pi\left(16 k^{1 / 2}\right)^{-1}-3\left(16 k^{1 / 2}\right)^{-1} \log \left[\left(1+k^{-1}\right)^{1 / 2}-1\right]\left[\left(1+k^{-1}\right)^{1 / 2}+1\right]^{-1} \\
& -3\left(8 k^{1 / 2}\right)^{-1} \arctan \left(1+k^{-1}\right)^{1 / 2}+\left(\frac{1}{4}\right)\left(2+k^{-1}\right)^{-1} k^{1 / 2}\left(1+k^{-1}\right)^{3 / 2} \\
I_{2} & =3\left(16 k^{1 / 2}\right)^{-1} \log \left[1-\left(1-k^{-1}\right)^{1 / 2}\right]\left[\left(1-k^{-1}\right)^{1 / 2}+1\right]^{-1} \\
& +3\left(8 k^{1 / 2}\right)^{-1} \arctan \left(1-k^{-1}\right)^{1 / 2}+4^{-1}\left(2-k^{-1}\right)^{-1}\left(1-k^{-1}\right)^{3 / 2} \\
& -3\left(16 k^{1 / 2}\right)^{-1} \log \left(k^{1 / 2}-1\right)\left(1+k^{1 / 2}\right)^{-1}-3\left(8 k^{1 / 2}\right)^{-1} \arctan k^{-1 / 2}+4^{-1}\left(1-k^{2}\right)^{-1}
\end{aligned}
$$

Analogously,

$$
\begin{aligned}
\int x^{-2}\left(x^{4}-b^{4}\right)^{-2} \mathrm{~d} x & =-5\left(16 b^{9}\right)^{-1} \log \left(|x-b||x+b|^{-1}\right) \\
& -5\left(8 b^{9}\right)^{-1} \arctan (x / b)-\left(4 b^{8}\right)^{-1}\left(5 x^{4}-4 b^{4}\right) x^{-1}\left(x^{4}-b^{4}\right)^{-1}+C
\end{aligned}
$$

and hence

$$
\begin{aligned}
I_{3} & =5\left(16 k^{9 / 2}\right)^{-1} \log \left[\left(1+k^{-1}\right)^{1 / 2}-1\right]\left[\left(1+k^{-1}\right)^{1 / 2}+1\right]^{-1} \\
& -5\left(8 k^{9 / 2}\right)^{-1}\left[(\pi / 2)-\arctan \left(1+k^{-1}\right)^{1 / 2}\right] \\
& +\left(4 k^{4}\right)^{-1}(k+1)^{-1 / 2}(2 k+1)^{-1}\left(k^{2}+10 k+5\right)
\end{aligned}
$$




$$
\begin{aligned}
I_{4} & =\left(4 k^{4}\right)^{-1}(k-1)^{-1 / 2}(2 k-1)^{-1}\left(k^{2}-10 k+5\right) \\
& +\left(4 k^{4}\right)^{-1}\left(4 k^{2}-5\right)\left(k^{2}-1\right)^{-1} \\
& -5\left(8 k^{9 / 2}\right)^{-1}-\left[\arctan \left(1-k^{-1}\right)^{1 / 2}-\arctan k^{-1 / 2}\right] \\
& -5\left(16 k^{9 / 2}\right)^{-1} \log \left[1-\left(1-k^{-1}\right)^{1 / 2}\right]\left[\left(1-k^{-1}\right)^{1 / 2}+1\right]^{-1} \\
& +5\left(15 k^{9 / 2}\right)^{-1} \log \left[k^{1 / 2}-1\right]\left[k^{1 / 2}+1\right]^{-1} .
\end{aligned}
$$

The stated estimates for $I_{1}, I_{2}, I_{3}, I_{4}$ are now evident. This proves (8.i).

(8.ii) (LEMMA) [20]. If $\phi \in L(G)$ and $\iint_{G} \phi \mathrm{d} t \mathrm{~d} x=0$, then for every $\psi \in L(G)$ such that $m \leqslant \psi \leqslant M$, we also have

$$
-2^{-1}(M-m) \iint_{G}|\phi| \mathrm{d} t \mathrm{~d} x \leqslant \iint \phi \psi \mathrm{d} t \mathrm{~d} x \leqslant 2^{-1}(M-m) \iint_{G}|\phi| \mathrm{d} t \mathrm{~d} x
$$

Indeed, if $G^{+}=[(t, x) \in G \mid \phi(t, x) \geqslant 0]$ and $G^{-}=G-G^{+}$, then

$$
\iint_{G^{+}} \phi \mathrm{d} t \mathrm{~d} x=-\iint_{G^{-}} \phi \mathrm{d} t \mathrm{~d} x=2^{-1} \iint_{G}|\phi| \mathrm{d} t \mathrm{~d} x
$$

and

$$
\begin{aligned}
\iint_{G} \phi \psi \mathrm{d} t \mathrm{~d} x & =\left(\iint_{G^{+}}+\iint_{G^{-}}\right) \phi \psi \mathrm{d} t \mathrm{~d} x \\
& \leqslant M \iint_{G^{+}} \phi \mathrm{d} t \mathrm{~d} x+m \iint_{G^{-}} \phi \mathrm{d} t \\
& =2^{-1}(M-m) \iint_{G}|\phi| \mathrm{d} t \mathrm{~d} x .
\end{aligned}
$$

This is the second inequality (8.4). Analogously we can prove the first inequality (8.4).

\section{A MEASURE THEORETICAL PROPERTY OF BOUNDED OPEN SETS}

We shall denote by $U\left(t_{0}, r\right)$ the closed ball in $\mathbb{R}^{\nu}$ of center $t_{0}$ and radius $r$. For any open bounded subset $G$ of $R^{\nu}$, let $D$ denote the diameter of $G$ and by $a$ the measure of $G$, thus $0<D<\infty, 0<a<\infty$.

(9.i) (LEMMA). Any open bounded subset $G$ of $\mathbb{R}^{\nu}$ has the following property $(P)$ : there is a function $k(r), 0 \leqslant r \leqslant D, k(0)=0, k(r)>0$ for $0<r \leqslant D, k(D)=a, k(r)$ depending on $G$ only, such that meas $\left[G \cap U\left(t_{0}, r\right)\right] \geqslant k(r)$ for all $t_{0} \in G$ and all $0 \leqslant r \leqslant D$.

Proof. Let us assume that this statement is not true. Then there is a set $G$ open and bounded, a sequence [ $t_{k}$ ] of points $t_{k} \in G$, and numbers $r_{0}>0, r_{k} \geqslant r_{0}>0$, such that meas $\left[G \cap U\left(t_{k}, r_{k}\right)\right] \rightarrow 0$ as $k \rightarrow \infty$. By an extraction and further relabeling, we may well assume that $t_{k} \rightarrow t_{0} \in \bar{G}, r_{k} \rightarrow \bar{r} \geqslant r_{0}>0$ as $k \rightarrow \infty$. Then we may well assume $\bar{r}=r_{0}$, and then

$$
t_{k} \rightarrow t_{0} \in \bar{G}, \quad r_{k} \rightarrow r_{0}>0, \quad \operatorname{meas}\left[G \cap U\left(t_{k}, r_{k}\right)\right] \rightarrow 0 \quad \text { as } k \rightarrow \infty .
$$

On the other hand $t_{k} \rightarrow t_{0}, r_{k} \rightarrow r_{0}$ implies that $U\left(t_{k}, r_{k}\right) \rightarrow U\left(t_{0}, r_{0}\right)$ as $k \rightarrow \infty$, hence $G \cap$ $U\left(t_{k}, r_{k}\right) \rightarrow G \cap U\left(t_{0}, r_{0}\right)$, and meas $\left[G \cap U\left(t_{k}, r_{k}\right)\right] \rightarrow 0=\operatorname{meas}\left[G \cap U\left(t_{0}, r_{0}\right)\right]$. But 
this is impossible, since $U\left(t_{0}, r_{0}\right)$ certainly contains interior points of $G$ and meas $[G \cap$ $\left.U\left(t_{0}, r_{0}\right)\right]>0$.

\section{REMARKS ON THE (LL) AND (*) CONDITIONS}

The Landesman and Lazer condition (LL), in its typical form, concerns problems of the type

$$
E x=\phi(t)+g(x), \quad t \in G,
$$

in the unknown $x(t), t \in G, x \in X$, where $G$ is a domain in $\mathbb{R}^{\nu}, \nu \geqslant 1$, say of finite measure $a=$ meas $G, 0<a<+\infty$, where $g: \mathbb{R} \rightarrow \mathbb{R}$ is a given nonlinear real valued function with $g(-\infty)=R^{-}, g(+\infty)=R^{+}$finite, say $g$ continuous and therefore bounded in $\mathbb{R}$, and wherc $\phi: G \rightarrow \mathbb{R}$ is a given measurable function on $G$, say bounded. Here, $E$ denotes a linear operator, say a differential operator in $G$ with homogeneous linear boundary conditions, and nontrivial ker $E$. We assume that a real Banach or Hilbert space $X$ of functions $x(t), t \in G$, has been chosen so that $D(E) \subset X \subset L_{2}(G)$, in particular ker $E \subset X \subset L_{2}(G)$.

Note that, for any real function $v(t), t \in G, v \in L_{1}(G)$, we may denote by $G^{+}$and $G^{-}$ the sets $G^{+}=[t \in G \mid v(t)>0], \quad G^{-}=[t \in G \mid v(t)<0], \quad$ and take $v^{+}=\int_{G^{+}} v \mathrm{~d} t, \quad v^{-}=$ $\int_{G^{-}}|v| \mathrm{d} t$.

The (LL)-condition in the space $X$ can be expressed by requiring that (LL): for any $v(t)$, $t \in G, v \in \operatorname{ker} E$, with $\|v\|_{X}=1$, then

$$
R^{-} v^{-}-R^{+} v^{+}<\int_{G} \phi(t) v(t) \mathrm{d} t<R^{+} v^{-}-R^{-} v^{+}
$$

By $(L L)_{w}$-condition we shall mean the same requirement with $\leqslant$ replacing both $<$ signs.

A slightly stronger requirement is the following condition (LL) $)_{\varepsilon}$ : there is some $\varepsilon>0$ such that for any $v(t), t \in G, v \in \operatorname{ker} E$, with $\|v\|_{X}=1$, then

$$
R^{-} v^{-}-R^{+} v^{+}+\varepsilon<\int_{G} \phi(t) v(t) \mathrm{d} t<R^{+} v^{-}-R^{-} v^{+}-\varepsilon
$$

In the same context, we formulate now the condition already mentioned in Sections 1 and 2 by requiring that (*): there are numbers $R_{0}>0, r>0$ such that, for all $\rho \geqslant R_{0}$, for all $v(t)$, $t \in G, v \in \operatorname{ker} E,\|v\|_{X}=1$, and any function $\sigma(t), t \in G,\|\sigma\|_{X} \leqslant r$, we have

$$
\left.\int_{G} \phi(t) v(t) \mathrm{d} t+\int_{G} g(\rho v(t)+\sigma(t)) v(t) \mathrm{d} t \geqslant 0 \quad \text { [ог } \leqslant 0\right] .
$$

In a number of applications, all with $\operatorname{dim} \operatorname{ker} E<\infty$, the implications (LL) $\Rightarrow(\mathrm{LL})_{\varepsilon} \Rightarrow(*)$ have been verified (cf. [4]). We shall discuss below the relationship between the conditions above under a variety of assumptions.

Let $G$ as before be a measurable subset of $R^{\nu}, \nu \geqslant 1$, with $0<a=$ meas $G<+\infty$.

(10.i) (LEMMA). For any given $v(t) \geqslant 0, t \in G, v \in L_{2}(G),\|v\|_{2}=1$, any $c>0$, and $E_{0}$ $=[t \in G, 0 \leqslant v(t) \leqslant c], \eta_{0}=$ meas $E_{0}$, we have

$$
\|v\|_{1} \leqslant c \eta_{0}+\left(a-\eta_{0}\right)^{1 / 2} .
$$

Indeed,

$$
\begin{aligned}
\|v\|_{1} & =\left(\int_{E_{0}}+\int_{G-E_{0}}\right)|v| \mathrm{d} t \leqslant c \text { meas } E_{0}+\left(\int_{G-E_{0}} \mathrm{~d} t\right)^{1 / 2}\left(\int_{G-E_{0}} v^{2} \mathrm{~d} t\right)^{1 / 2} \\
& \leqslant c \eta_{0}+\left(a-\eta_{0}\right)^{1 / 2}
\end{aligned}
$$


(10.ii) Given $\varepsilon>0$ there is a number $\delta>0$ such that for any $v(t), t \in G, v \in L_{2}(G),\|v\|_{2}$ $=1$, such that, for $E_{0}=[t \in G,|v(t)| \leqslant \delta], \eta_{0}=$ meas $E_{0}$, we have $a-\delta \leqslant \eta_{0} \leqslant a$, then we also have $\|v\|_{1} \leqslant \varepsilon$.

Indeed, $\|v\|_{1}$ and $\|v\|_{2}$ are also the norms of the nonnegative function $|v(t)|, t \in G,|v| \in$ $L_{2}(G)$, with $L_{2}$-norm one. Take $\delta>0$ so that $a \delta+\delta^{1 / 2} \leqslant \varepsilon$. Then, by (10.i) with $c=\delta$ we also have $\|v\|_{1} \leqslant \delta \eta_{0}+\left(a-\eta_{0}\right)^{1 / 2} \leqslant a \delta+\delta^{1 / 2} \leqslant \varepsilon$.

We shall consider below sequences $(S)$ of functions $v_{n}(t), t \in G, v_{n} \in L_{2}(G),\left\|v_{n}\right\|_{2}=1$, $n=1,2, \ldots$, and constants $c_{n}>0, \eta_{n}=$ meas $E_{0 n}, E_{0 n}=\left[t \in G|| v_{n}(t) \mid<c_{n}\right]$. Then, $c_{n} \rightarrow 0, \eta_{n} \rightarrow a$ implies $\left\|v_{n}\right\|_{1} \rightarrow 0$ as $n \rightarrow \infty$, as immediate corollary of (10.i).

(10.iii) A relation (LL) $)_{\varepsilon}$ is impossible if ker $E$ contains sequences $(S)$, that is, sequences of functions $v_{n}(t), t \in G, v_{n} \in L_{2}(G),\left\|v_{n}\right\|_{2}=1,\left\|v_{n}\right\|_{1} \rightarrow 0$ as $n \rightarrow \infty$.

Indeed, then $v_{n}^{+}, v_{n}^{-} \leqslant\left\|v_{n}\right\|_{1}$, hence $v_{n}^{+} \rightarrow 0, v_{n}^{-} \rightarrow 0$, and, $R^{+} v_{n}^{+}, R^{-} v_{n}^{+} \rightarrow 0$ as $n \rightarrow \infty$. Then a relation $(\mathrm{LL})_{\varepsilon}$ is not satisfied for $n$ sufficiently large.

(10.iv) (LEMMA). Let $G$ be a measurable subset of $\mathbb{R}^{\nu}, \nu \geqslant 1$, with finite measure $0<a=$ meas $G<+\infty$, and let $1 \leqslant m=\operatorname{dim} \operatorname{ker} E<\infty$. Let us assume that there is some $\lambda_{0} \geqslant 0$ such that $v(t), t \in G, v \in \operatorname{ker} E, v \in L_{2}(G),\|v\|_{2}=1$, implies $v(t)=0$ at most in a subset $E_{0}$ of $G$ with meas $E_{0} \leqslant \lambda_{0}$. Then, given $\varepsilon>0$ there is some constant $c>0$ such that meas $[t$ $\in G|| v(t) \mid \leqslant c] \leqslant \lambda_{0}+\varepsilon$ for all $v$ as above.

Proof. Let $w_{1}, \ldots, w_{m}$ denote any orthogonal basis for ker $E$. Then, for every $v \in$ ker $E$, we have $v=b_{1} w_{1}+\ldots+b_{m} w_{m}, b_{i}=\left(v, w_{i}\right), i=1, \ldots, m, b=\left(b_{1}, \ldots, b_{m}\right)$, and $\|v\|_{2}=|b|$ where $|b|$ is the Euclidean norm of $b$ in $R^{m}$.

If the statement above is not true, then there is a number $\varepsilon_{0}>0$ and sequences $c_{n}>0, v_{n}(t), t \in G, v_{n} \in \operatorname{ker} E,\left\|v_{n}\right\|_{2}=1$, with $\eta_{n} \geqslant \lambda_{0}+\varepsilon_{0}, c_{n} \rightarrow 0, \eta_{n}=$ meas $E_{n}, E_{n}=[t \in$ $\left.G|| v_{n}(t) \mid \leqslant c_{n}\right]$. Then $v_{n}=b_{n 1} w_{1}+\ldots+b_{n m} w_{m}$ for $\left|b_{n}\right|=1, b_{n}=\left(b_{n 1}, \ldots, b_{n m}\right)$, and there is a subsequence, say still $[n]$, such that $b_{n} \rightarrow b,|b|=1, b=\left(b_{1}, \ldots, b_{n}\right)$, or $b_{n i} \rightarrow b_{i}, i=$ $1, \ldots, m$, as $n \rightarrow \infty$. Let $v(t)=b_{1} w_{1}+\ldots+b_{m} w_{m}, t \in G$, and certainly $v \in$ ker $E$. First, assume that $w_{1}, \ldots, w_{m}$ are bounded in $G$, say $\left|w_{i}(t)\right| \leqslant M, t \in G, i=1, \ldots, m$. Then, for $\sigma_{n}=\left|b-b_{n}\right|$, we have $\sigma_{n} \rightarrow 0$ and $|v(t)| \leqslant\left|v_{n}(t)\right|+\left|v(t)-v_{n}(t)\right| \leqslant c_{n}+M \sigma_{n}$ for all $t \in E_{n}$, where $c_{n}+M \sigma_{n} \rightarrow 0$ as $n \rightarrow \infty$, and meas $E_{n} \geqslant \lambda_{0}+\varepsilon_{0}$. Here $v_{n} \rightarrow v$ uniformly, and thus $v(t)=0$ in some set $E_{0}$ of measure $\geqslant \lambda_{0}+\varepsilon_{0}$, a contradiction.

If the functions $w_{i}(t)$ are only in $L_{2}(G)$, then we take $N>0$ so large that for each $i=$ $1, \ldots, m$, the set $F_{i}=\left[t \in G|| w_{i}(t) \mid \geqslant N\right]$ has measure meas $F_{i}<\varepsilon_{0} / 2 m$, and we take $F=$ $F_{1} \cup \ldots \cup F_{m}$, meas $F<\varepsilon_{0} / 2$. Now we repeat the argument above, with $E_{n}^{\prime}=E_{n}-F$, meas $E_{n}^{\prime} \geqslant \lambda_{0}+\varepsilon_{0} / 2$, and $N$ replacing $M$.

Here are a few examples concerning conditions (LL) and (*).

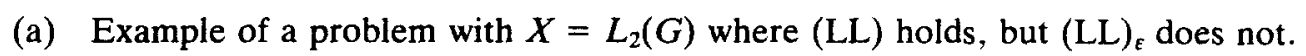

Consider the problem

$$
\begin{aligned}
x_{\pi}-x_{\xi \xi} & =f(t, \xi)+g(x(t, \xi)), \quad(t, \xi) \in \mathbb{R}^{2}, \\
x(t+2 \pi, \xi) & =x(t, \xi)=x(t, \xi+2 \pi),
\end{aligned}
$$




$$
f(t+2 \pi, \xi)=f(t, \xi)=f(t, \xi+2 \pi)
$$

for which ker $E$ contains all trigonometrical polynomial generated by the usual exponentials $\mathrm{e}^{i(k t+l \xi)}, k, l=0, \pm 1, \pm 2, \ldots, k^{2}=l^{2}$, and limit elements in $L_{2}(G), G=[0,2 \pi]^{2}$. Let $g$ : $R \rightarrow R$, be continuous with limits $R^{+}=g(+\infty)>0, \quad R^{-}=\mathrm{g}(-\infty)<0$, take $\mu=$ $\min \left[R^{+},-R^{-}\right]$, and notc that for $|f(t, \xi)| \leqslant M<\mu$, relation (LL) ccrtainly holds since

$$
\begin{aligned}
& R^{+} v^{-}-R^{-} v^{+}-\int_{G} f v \mathrm{~d} t \geqslant \mu\left(v^{-}+v^{+}\right)-M\|v\|_{1}=(\mu-M)\|v\|_{1}>0 \\
& \int_{F} f v \mathrm{~d} t+R v^{+}-R^{-} v^{-} \geqslant \mu\left(v^{+}+v^{-}\right)-M\|v\|_{1}=(\mu-M)\|v\|_{1}>0 .
\end{aligned}
$$

For every $n$, let $\psi_{n}(\xi),-\infty<\xi<+\infty$, be a trigonometrical polynomial with $\left\|\psi_{n}(\xi)\right\|_{2}=$ $(2 \pi)^{-1 / 2}$, (square norm in $[0,2 \pi]$ ), with $0<\psi_{n}(\xi) \leqslant 1 / n$ for $1 / n \leqslant \xi \leqslant 2 \pi-1 / n$, and $\psi_{n}(\xi) \geqslant 1 / n$ for $0 \leqslant \xi \leqslant 1 / n, 2 \pi-1 / n<\xi \leqslant 2 \pi$. Note that $v_{n}(t, \xi)=\psi_{n}(\xi-t)$ is a trigonometrical polynomial in $R^{2}$, is periodic of period $2 \pi$ both in $t$ and $\xi$, is an element of ker $E$, and its square norm in $G=[0,2 \pi]$, is $\left\|v_{n}\right\|_{2}^{2}=2 \pi\left\|\psi_{n}\right\|_{2}^{2}=2 \pi(1 / 2 \pi)=1$. Moreover, $0<v_{n} \leqslant 1 / n$ everywhere in $G$ but a diagonal strip $G-E_{n}$ where $v_{n} \geqslant 1 / n$ and meas $\left(G-E_{n}\right)=(2 / n) 2 \pi=$ $4 \pi / n$. In other words, $\left[v_{n}\right]$ is a sequence $(S)$ in $G$. Condition (LL) $)_{\varepsilon}$ is then impossible.

This example can be modified in such a way that $(*)$ also does not hold. Let us assume $g$ : $\mathbb{R} \rightarrow \mathbb{R}$ to be strictly increasing with a unique zero at $x=2 \delta>0$, or $g(2 \delta)=0,0<g(x)<$ $R$ for $x>2 \delta, g(+\infty)=R, r<g(x)<0$ for $x<2 \delta, r=g(-\infty), R>0, r<0$. Then, if $\tau=$ $-g(\delta)$, then $\tau>0$, and $g(x) \leqslant-\tau$ for $x \leqslant \delta$. For the same functions $v_{n}(t, \xi)$ of No. 3 , and 0 $\leqslant \rho \leqslant n \delta$, we have

$$
\begin{aligned}
& 0<\rho v_{n}(t, \xi) \leqslant(n \delta)(1 / n)=\delta, \quad g\left(\rho v_{n}(t, \xi)\right) \leqslant-\tau \quad \text { if }(t, \xi) \in E_{n}, \\
& 0<\rho v_{n}(t, \zeta), \quad g\left(\rho v_{n}(t, \xi)\right) \leqslant R \quad \text { if }(t, \xi) \in G-E_{n},
\end{aligned}
$$

so that, for $|f| \leqslant M$ and for $n$ so large that $M\left\|_{v_{n}}\right\|_{1}<\pi^{2} \tau, 2 R / n<\pi \tau, 4 / n<\pi$, then

$$
\begin{aligned}
\int_{G} f v_{n} \mathrm{~d} t \mathrm{~d} \zeta & +\int_{G} g\left(\rho v_{n}(t, \zeta)\right) v_{n}(t, \zeta) \mathrm{d} t \mathrm{~d} \zeta \\
& =\int_{G} f v_{n} \mathrm{~d} t \mathrm{~d} \zeta+\int_{E_{n}} g\left(\rho v_{n}\right) v_{n} \mathrm{~d} t \mathrm{~d} \zeta+\int_{G-E_{n}} g\left(\rho v_{n}\right) v_{n} \mathrm{~d} t \mathrm{~d} \zeta \\
& \leqslant M\left\|v_{n}\right\|_{1}-\tau \operatorname{meas} E_{n}+R \operatorname{meas}\left(G-E_{n}\right) \\
& \leqslant \pi^{2} \tau-\tau\left(4 \pi^{2}-4 \pi / n\right)+R(2 \pi / n) \\
& \leqslant-4 \pi^{2} \tau+\pi^{2} \tau+\pi^{2} \tau+\pi^{2} \tau=-\pi^{2} \tau
\end{aligned}
$$

and this holds for all $n$ sufficiently large and all $0 \leqslant \rho \leqslant n \delta$.

On the other hand, for $v=(2 \pi)^{-1}$ in $G,\|v\|_{2}=1, M+\varepsilon<R^{+}$for some $\varepsilon>0$, and $\rho$ so large that $g(\rho)>M+\varepsilon / 2$, we have $\int_{G}[f v+g(\rho v) v] \mathrm{d} t \mathrm{~d} \xi>(2 \pi)(g(\rho)-M) \geqslant(2 \pi)(\varepsilon / 2)$. This shows that there is no $R_{0}$ such that the $(*)$ relation holds for all $\rho>R_{0}$ and all $v \in$ ker $E$ with $\|v\|_{2}=1$.

(b) Example of problem with $X=L_{2}(G)$ for which (LL) wolds but the problem has no solutions. 
Take $E \equiv 0$ thus $\operatorname{ker} E=L_{2}(G)$, take $\phi(t)=+1$ in a set $E_{1} \subset G, \phi(t)=-1$ in a set $E_{2} \subset$ $G$, meas $E_{1}>0$, meas $E_{2}>0, E_{1} \cup E_{2}=G, E_{1} \cap E_{2}=\varnothing$, and take $g: \mathbb{R} \rightarrow \mathbb{R}$ continuous, with $g(+\infty)=1, g(-\infty)=-1,-1<g(x)<1$ for all $x \in \mathbb{R}$. Then for every $v(t), t \in G, v \in$ $L_{2}(G),\|v\|_{2}=1$ we certainly have $1-\phi(t) \operatorname{sgm} v(t) \geqslant 0$ in $G$, and

$$
R^{+} v^{+}-R^{-} v^{-}-\int_{G} \phi(t) v(t) \mathrm{d} t=\int_{G}[1-\phi(t) \operatorname{sgm} v(t)]|v(t)| \mathrm{d} t \geqslant 0 .
$$

However, the problem $E x=0=\phi(t)+g(x(t))$ has no solution, since everywhere in $G$ we have $\phi(t)= \pm 1,-1<g(x(t))<1$, or $\phi+g \neq 0$.

\section{SUFFICIENT CONDITIONS FOR PROPERTY (*)}

(11.i) THEOREM. Let $r>0$ be a given number. Let $g: \mathbb{R} \rightarrow \mathbb{R}$ be a continuous function with finite limits $R^{+}=g(+\infty), R^{-}=g(-\infty)$. Then, given $\varepsilon>0$ there is $R_{0}>0$ such that, for $\rho \geqslant R_{0}$, for any function $v(t), t \in G, v \in L_{2}(G),\|v\|_{2}=1$, and any function $\sigma(t), t \in G$, $\sigma \in L_{2}(G),\|\sigma\|_{2} \leqslant r$, we have

$$
\left|\int_{G} g(\rho v(t)+\sigma(t)) v(t) \mathrm{d} t-R^{+} v^{+}+R^{-} v^{-}\right| \leqslant \varepsilon .
$$

Proof. Let $0<a=$ meas $G<+\infty$, and take $C$ such that $|g(x)| \leqslant C$ for all $x \in \mathbb{R}$. Let $\eta>0$ be a constant such that $C \eta a \leqslant \varepsilon / 8$.

Let $N>\eta$ be a constant such that $C N^{-1} r \leqslant \varepsilon / 8$.

Let $\Lambda \geqslant 0$ be a constant such that $\left|g(x)-R^{+}\right| \leqslant \varepsilon / 8 a$ for all $x \geqslant \Lambda$ and $\left|g(x)-R^{-}\right| \leqslant$ $\varepsilon / 8 \mathrm{a}$ for all $x \leqslant-\Lambda$.

Let $R_{0}$ be any constant $R_{0} \geqslant \eta^{-1}(\Lambda+N)$. Clearly $R_{0}$ depends only on $G, g$ and $E$.

For any function $v(t), t \in G, v \in L_{2}(G),\|v\|_{2}=1$, let $E_{0}=[t \in G|| v(t) \mid \leqslant \eta]$ and $E_{1}=$ $G-E_{0}$. For any function $\sigma(t), t \in G, \sigma \in L_{2}(G),\|\sigma\|_{2} \leqslant r$ let $F=[t \in G|| \sigma(t) \mid \geqslant N]$. Then $N^{2}$ meas $F \leqslant \int_{F} \sigma^{2} \mathrm{dt} \leqslant \mathrm{r}^{2}$, or meas $F \leqslant N^{-2} r^{2}$. Then

$$
\begin{aligned}
\left|\int_{F} g(\rho v(t)+\sigma(t)) v(t) \mathrm{d} t\right| & \leqslant C \int_{F}|v(t)| \mathrm{d} t \leqslant C(\text { meas } F)^{1 / 2}\|v\|_{2} \\
& \leqslant C N^{-1} r \leqslant \varepsilon / 8, \\
\left|\int_{E_{0}} g(\rho v(t)+\sigma(t)) v(t) \mathrm{d} t\right| & \leqslant C \eta \text { meas } E_{0} \leqslant C \eta a \leqslant \varepsilon / 8 .
\end{aligned}
$$

Note that

$$
\begin{aligned}
v^{+} & =\int_{G^{+}}|v(t)| \mathrm{d} t=\int_{G^{+}} v(t) \mathrm{d} t \\
& =\left(\int_{F \cap G^{+}}+\int_{(G-F) \cap E_{0} \cap G^{+}}+\int_{(G-F) \cap E_{1} \cap G^{+}}\right) v(t) \mathrm{d} t .
\end{aligned}
$$

and an analogous decomposition holds for $v^{-}=\int_{G}|v(t)| \mathrm{d} t=-\int_{G^{-}} v(t) \mathrm{d} t$, where the last member must be taken with a sign minus. Now for $\rho \geqslant R$ and $t \in(G-F) \cap E_{1} \cap G^{+}$ 
we have $v(t) \geqslant \eta>0, \rho v(t)+\sigma(t) \geqslant R_{0} \eta-N \geqslant(\Lambda+N)-N=\Lambda$, and $\mid g(\rho v(t)+\sigma(t))$ $-R^{+} \mid<\varepsilon / 8 a$. Then

$$
\begin{aligned}
& \left|\int_{(G-F) \cap E_{1} \cap G^{+}} g(\rho v(t)+\sigma(t)) v(t) \mathrm{d} t-R^{+} v^{+}\right| \\
& \quad=\mid \int_{(G-F) \cap E_{1} \cap G^{+}}\left[g(\rho v(t)+\sigma(t))-R^{+}\right] v(t) \mathrm{d} t+R^{+} \int_{F \cap G^{+}} v(t) \mathrm{d} t \\
& \quad-R^{+} \int_{\left(G-\cap \cap E_{1} \cap G^{+}\right.} v(t) \mathrm{d} t \\
& \quad \leqslant(\text { meas } G)(\varepsilon / 8 a)+C(\text { meas } F)^{1 / 2}\|v\|_{2}+C(\text { meas } G) \eta \\
& \quad \leqslant a(\varepsilon / 8 a)-C N^{-1} r+C a \eta \leqslant \varepsilon / 8+\varepsilon / 8+\varepsilon / 8=3 \varepsilon / 8
\end{aligned}
$$

Analogously we have, for $\rho \geqslant R_{0}$,

$$
\left|\int_{(G-F) \cap E_{1} \cap G^{-}} g(\rho v(t)+\sigma(t)) v(t) \mathrm{d} t+R^{-} v^{-}\right| \leqslant 3 \varepsilon / 8
$$

Combining all relations (11.2)-(11.5) we see that all $\rho \geqslant R_{0}$, and $v$ and $\sigma$ as stated, we have

$$
\left|\int_{G} g(\rho v(t)+\sigma(t)) v(t) \mathrm{d} t-R^{+} v^{+}+R^{-} v^{-}\right| \leqslant \varepsilon / 8+\varepsilon / 8+3 \varepsilon / 8+3 \varepsilon / 8=\varepsilon .
$$

Remark. Statement (11.i) holds for arbitrary elements $v \in L_{2}(G)$ in the same form with (11.1) replaced by

$$
\left|\int_{G} g(\rho v(t)+\sigma(t)) v(t) \mathrm{d} t-R^{+} v^{+}+R^{-} v^{-}\right| \leqslant \varepsilon\|v\|_{L_{1}}
$$

and the analogous relation with $R^{+} v^{+}-R^{-} v^{-}$replaced by $R^{+} v^{-}-R^{-} v^{+}$follows by exchanging $v$ with $-v$.

(11.ii) CoRollary. With $D(E) \subset X \subset L_{2}(G),\|v\|_{L_{2}} \leqslant c\|v\|_{X}$ for some constant $c>0$, then condition (LL) $)_{\varepsilon}$ implies (*).

Indeed, given $r>0$ we choose $R_{0}>0$ so that, for $\rho \geqslant R_{0}$ relation (11.6) holds with $2^{-1} \varepsilon a^{-1 / 2} c^{-1}$, instead of $\varepsilon, a=$ meas $G, 0<a<+\infty$, hence

$$
\begin{aligned}
& \left|\int_{G} g(\rho v(t)+\sigma(t)) v(t) \mathrm{d} t-R^{+} v^{+}+R^{-} v^{-}\right|<\left(2^{-1} \varepsilon a^{-1 / 2} c^{-1}\right)\|v\|_{L_{1}} \\
& \quad \leqslant\left(2^{-1} \varepsilon a^{-1 / 2} c^{-1}\right) a^{1 / 2}\|v\|_{L_{2}} \leqslant\left(2^{-1} \varepsilon a^{-1 / 2} c^{-1}\right) a^{1 / 2} c\|v\|_{X}=\varepsilon / 2
\end{aligned}
$$

for $\|v\|_{X}=1$. Then

$$
R^{-} v^{+}-R^{+} v^{-}+\varepsilon<\int_{G} \phi v \mathrm{~d} t<R^{+} v^{+}-R^{-} v^{-}-\varepsilon
$$


implies

$$
\int_{G}[\phi(t)+g(\rho v(t)+\sigma(t))] v(t) \mathrm{d} t \geqslant \varepsilon / 2>0 .
$$

(11.iii) If $R^{+}>0, R^{-}<0$, and if $|\phi(t)| \leqslant M<\mu=\min \left[R^{+},-R^{-}\right]$, then for any $v \in L_{1}(G)$, $\|v\|_{L_{1}}>0$, we have

$$
\left|\int_{G} \phi v \mathrm{~d} t\right| \leqslant M\|v\|_{L_{1}}<\mu\|v\|_{L_{1}} \leqslant R^{+} v^{+}-R^{-} v^{-}
$$

Thus, if $D(E) \subset X \subset L_{1}(G)$ and there is a constant $\gamma>0$ such that $\|v\|_{X}=1, v \in$ ker $E$ implies $\|v\|_{L_{1}} \geqslant \gamma$, then for the same $v$ we also have

$$
\left|\int_{G} \phi v \mathrm{~d} t\right|<R^{+} v^{+}-R^{-} v^{-}-\varepsilon
$$

with $\varepsilon=(\mu-M) \gamma$.

(11.iv) (LemMA). Let $G$ be a measurable subset of $\mathbb{R}^{\nu}, 0<a=$ meas $G<+\infty$, and let $c$ be any constant, $0<c<a^{-1 / 2}$. Then for any measurable essentially bounded function $v(t)$, $t \in G,\|v\|_{2}=1$, and $\mu=\operatorname{ess} \sup [|v(t)|, t \in G], E_{0}=[t \in G|| v(t) \mid \leqslant c], \eta_{0}=$ meas $E_{0}$, we have $\mu \geqslant a^{-1 / 2}$, and $0 \leqslant \eta_{0} \leqslant a-\mu^{-2}\left(1-a c^{2}\right)$.

Indeed, $1=\|v\|_{2}^{2} \leqslant a \mu^{2}$, or $\mu \geqslant a^{-1 / 2}$. On the other hand,

$$
1=\|v\|_{2}^{2} \leqslant \eta_{0} c^{2}+\left(a-\eta_{0}\right) \mu^{2} \leqslant a c^{2}+\left(a-\eta_{0}\right) \mu^{2},
$$

or

$$
\eta_{0} \leqslant a-\mu^{-2}\left(1-a c^{2}\right)
$$

where now $a \mu^{2} \geqslant 1>1-a c^{2}$ implies $\mu^{-2}\left(1-a c^{2}\right)<a$.

(11.v) Let $G$ be an open bounded connected subset $G$ with diameter $D$, and $0<a=$ meas $\bar{G}<+\infty$. Let $\omega(\zeta), 0 \leqslant \zeta<+\infty$, be a modulus of continuity, that is, a continuous increasing real function, with $\omega(0)=0$. Then, there are constants $\Gamma_{0}>0, \gamma_{0}>0$, which depend only on $G$ and the function $\omega$, such that, if $v(t), t \in \bar{G}$, is any continuous function on $G$ with $\|v\|_{2}$ $=1$ and modulus of continuity $\omega(\zeta)$, that is, $\left|v(t)-v\left(t^{\prime}\right)\right| \leqslant \omega\left(\left|t-t^{\prime}\right|\right)$ for all $t, t^{\prime} \in \bar{G}$, and $E_{1}=\left[t \in G|| v(t) \mid \geqslant \Gamma_{0}\right], \eta_{1}=$ meas $E_{1}$, we have $\eta_{1}=$ meas $E_{1} \geqslant \gamma_{0}>0$.

Proof. For any $c>0$ let $E_{0}=[t \in G|| v(t) \mid<c], \eta_{0}=\operatorname{meas} E_{0}$, and let $\mu=\max |v(t)|$ in $G$. Let $t_{0}$ be any point of $\bar{G}$ where $\left|v\left(t_{0}\right)\right|=\mu$. We know already that $\mu \geqslant a^{-1 / 2}$. For $c>0$ such that $0<c<(2 a)^{-1 / 2}$ we know from $(11 . i v)$ that $\eta_{0} \leqslant a-\mu^{-2}\left(1-a c^{2}\right)$. Thus, if $a^{-1 / 2} \leqslant \mu \leqslant$ $\left[2\left(1-a c^{2}\right) a^{-1}\right]^{1 / 2}$, then $\mu^{2} \leqslant 2\left(1-a c^{2}\right) a^{-1}$, or $\mu^{-2}\left(1-a c^{2}\right) \geqslant a / 2$, and $\eta_{0} \leqslant a-a / 2=a / 2$. Hence, for $\Gamma_{0}=c, \eta_{1}+\eta_{0}=a$, implies $\eta_{1} \geqslant a / 2$.

If $\mu \geqslant\left[2\left(1-a c^{2}\right) a^{-1}\right]^{1 / 2}$, then $\mu \geqslant\left[\left(1-a c^{2}\right) a^{-1}\right]^{1 / 2} \geqslant a^{-1 / 2} \geqslant(2 a)^{-1 / 2}>c>0$. Let $r>0$ be any number such that $\omega(\rho) \leqslant\left[2\left(1-a c^{2}\right) a^{-1}\right]^{1 / 2}-c$ for all $0 \leqslant \rho \leqslant r$. Then, for $t \in G \cap$ $U\left(t_{0}, r\right)$ we have

$$
\begin{aligned}
|v(t)| & \geqslant\left|v\left(t_{0}\right)\right|-\omega\left(\left|t-t_{0}\right|\right) \geqslant \mu-\omega(r) \\
& \geqslant\left[2\left(1-a c^{2}\right) a^{-1}\right]^{1 / 2}-\left\{\left[2\left(1-a c^{2}\right) a^{-1}\right]^{1 / 2}-c\right]=c>0
\end{aligned}
$$


that is, $G \cap U\left(t_{0}, r\right) \subset E_{1}$, and meas $E_{1} \geqslant$ meas $\left[G \cap U\left(t_{1}, r\right)\right] \geqslant k(r)>0$ where $k(r)$ is the function defined in $(9 . i)$ for the open set $G$.

In either case we have $|v(t)| \geqslant c=\Gamma_{0}$ in a set $E_{1}$ with meas $E_{1}=\min [a / 2, k(r)]=\gamma_{0}>0$.

(11.vi) TheOREM. Let $G$ be a measurable subset of $\mathbb{R}^{v}$ with $0<a=$ meas $G<+\infty$. Let $f(t, x)$ be a continuous function on $G \times \mathbb{R}$ such that for suitable constants $b, c, \lambda, B, C, 0<b<c$, $0<B<C$, we have

$$
\begin{aligned}
&|f(t, x)| \leqslant C \quad \text { for all } t \in G, \quad x \in \mathbb{R} \\
&|f(t, x)| \leqslant A \quad \text { for }|x| \leqslant b, \quad t \in G ; \\
& f(t, x) \geqslant 0 \quad \text { for } x \geqslant b, \quad f(t, x) \leqslant 0 \quad \text { for } x \leqslant-b, \quad t \in G ; \\
& f(t, x) \geqslant B \quad \text { for } x \geqslant c, \quad f(t, x) \leqslant-B \quad \text { for } x \leqslant-c, \quad t \in G .
\end{aligned}
$$

Let $\{v\}$ be a collection of functions $v(t), t \in G, v \in L_{2}(G)$, $\|v\|_{2}=1$, with the following property $(Q)$ : for given $\gamma>0, \Gamma>0$, and any $v \in\{v\}$, if $E_{1}=[t \in G|| v(t) \mid \geqslant \Gamma]$, then meas $E_{1} \geqslant \gamma$.

Let $\varepsilon$ be a given number, and assume $0<\varepsilon<\gamma / 3, A \leqslant a^{-1 / 2} B \varepsilon$. Then there are numbers $r>0, R_{0}>0$ such that for any $\rho \geqslant R_{0}, v \in\{v\}$, and any $\sigma(t), \sigma \in L_{2}(G),\|\sigma\|_{2} \leqslant r$, we have

$$
\int_{G} f(t, \rho v(t)+\sigma(t)) v(t) \mathrm{d} t \geqslant B(\gamma-3 \varepsilon)>0 .
$$

For $\varepsilon=\gamma / 3$, we have $\int_{G} f v \mathrm{~d} t \geqslant 0$.

Proof. For any $N>0$ let $F=[t \in G|| \sigma(t) \mid \geqslant N]$. Then $N^{2}$ meas $F \leqslant\|\sigma\| \frac{2}{2} \leqslant r^{2}$, or meas $F \leqslant N^{-2} r^{2}$. Let us assume that

Then

$$
C N^{-1} r \leqslant B \varepsilon, \quad N^{-2} r^{2} \leqslant \varepsilon, \quad N \leqslant b / 2 .
$$

$$
\begin{aligned}
\left|\int_{F} f(t, \rho v(t)+\sigma(t)) v(t) \mathrm{d} t\right| & \leqslant C \int_{F}|v(t)| \mathrm{d} t \leqslant C(\text { meas } F)^{1 / 2}\|v\|_{2} \\
& \leqslant C N^{-1} r \leqslant B \varepsilon .
\end{aligned}
$$

Let $K_{1}=[t \in G|| \rho v(t)+\sigma(t)|\leqslant b| v,(t) \mid \leqslant \Gamma]$. Then, for $t \in K_{1}-F$ we have $|\rho v(t)+\sigma(t)| \leqslant$ $b,|f(t, \rho v(t)+\sigma(t))| \leqslant A$, and

$$
\begin{aligned}
\left|\int_{K_{1}-F} f(t, \rho v(t)+\sigma(t)) v(t) \mathrm{d} t\right| & \leqslant A \int_{K_{1}-F}|v(t)| \mathrm{d} t \leqslant A(\operatorname{meas} G)^{1 / 2}\|v\|_{2} \\
& \leqslant A a^{1 / 2} \leqslant B \varepsilon .
\end{aligned}
$$

Let $K_{2}=[t \in G|| \rho v(t)+\sigma(t)|\geqslant b| v,(t) \mid \leqslant \Gamma]$. Then, for $t \in K_{2}-F$ we have $|\rho v(t)+\sigma(t)| \geqslant b$, $|\sigma(t)| \leqslant N \leqslant b / 2$. Hence $\rho|v(t)| \geqslant b-b / 2=b / 2$. Consequently, $\rho v(t)+\sigma(t)$ and $\rho v(t)$, that is $v(t)$, have the same sign and $f(t, \rho v(t)+\sigma(t))$ has the same sign as $v(t)$. Then

$$
\int_{K_{2}-F} f(t, \rho v(t)+\sigma(t)) v(t) \mathrm{d} t \geqslant 0 .
$$

Let $E_{1}=[t \in G,|v(t)| \geqslant \Gamma]$. Then, meas $\left(E_{1},-F\right) \geqslant$ meas $E_{1},-$ meas $F \geqslant \gamma-\varepsilon$. Take $R_{0} \geqslant$ $\Gamma^{-1}(c+N)$, and note that, for $\rho \leqslant R_{0}, t \in E_{1}-F$, we have $\rho|v(t)| \geqslant R_{0} \Gamma \geqslant c+N$, 
$|\sigma(t)| \leqslant N,|\rho v(t)+\sigma(t)| \geqslant c+N-N=c . \rho v(t)+\sigma(t)$ has the same sign as $v(t)$, and the same sign as $f(t, \rho v(t)+\sigma(t))$. Also, $f \geqslant B$ if $v \geqslant \Gamma>0$, and $f \leqslant-B$ if $v \leqslant-\Gamma<0$. Then

Now

$$
\int_{E_{1}-F} f(t, \rho v(t)+\sigma(t)) v(t) \mathrm{d} t \geqslant B \text { meas }\left(E_{1}-F\right) \geqslant B(\gamma-\varepsilon) .
$$

$$
\begin{aligned}
\int_{G} f v \mathrm{~d} t & =\left(\int_{F}+\int_{K_{1}-F}+\int_{K_{2}-F}+\int_{E_{1}-F}\right) f v \mathrm{~d} t \\
& \geqslant-B \varepsilon-B \varepsilon+0+B(\gamma-\varepsilon)=B(\gamma-3 \varepsilon) .
\end{aligned}
$$

Relations (11.7) can easily be satisfied by taking $N \leqslant b / 2$, and $r \leqslant \min \left[\varepsilon^{1 / 2} N, B \varepsilon C^{-1} N\right]$.

Remark. Let $G$ be any bounded open subset of $\mathbb{R}^{\nu}, \nu \geqslant 1$. Then property $(P)$ of $(9 . i)$ holds. Let the linear operator $E$ be given in $G$, and let us assume that the Hilbert spaces $X, Y$ have been selected so that $\|x\|_{L_{2}} \leqslant c\|x\|_{X}$ for $x \in X$, and so that the elements $x$ of the unit ball in $X$ are continuous on $G$ with modulus of continuity $\omega(\zeta)$. Then, by $(11 . v)$, numbers $\Gamma_{0}>0$, $\gamma_{0}>0$ can be determined so that $v \in L_{2}(G),\|v\|_{2}=1, E_{1}=\left[t \in G \| v(t) \mid \geqslant \Gamma_{0}\right], \eta_{1}=$ meas $E_{1}$, implies $\eta_{1} \geqslant \gamma_{0}$. Finally, by (11.vi) with $0<\varepsilon<\gamma_{0} / 3, A \leqslant a^{-1 / 2} B \varepsilon$, we can determine $R_{0}, r>0$ so that relation (*) holds for $\|v\|_{2}=1, \rho \geqslant R_{0},\|\sigma\|_{2} \leqslant r$.

In the case $f(t, x)=\phi(t)+g(x)$ with $g$ continuous in $\mathbb{R}$ and

then

$$
\begin{aligned}
|g(x)| & \leqslant C^{\prime} \quad \text { for all } x \in \mathbb{R}, \\
|g(x)| & \leqslant \beta \quad \text { for }|x| \leqslant b, \\
g(x) & \geqslant \nu \quad \text { for } x \geqslant b, \quad g(x) \leqslant-\nu \quad \text { for } x \leqslant-b, \\
g(x) & \geqslant B^{\prime} \text { for } x \geqslant c, \quad g(x) \leqslant-B^{\prime} \quad \text { for } x \leqslant-c, \\
|\phi(t)| & \leqslant \nu \quad \text { for all } t \in G,
\end{aligned}
$$

$$
\begin{aligned}
|f(t, x)| & =|\phi(t)+g(x)| \leqslant \nu+C^{\prime}=C \quad \text { for all }(t, x) \in G \times \mathbb{R}, \\
|f(t, x)| & =|\phi(t)+g(x)| \leqslant \nu+\beta=A \quad \text { for }|x| \leqslant b, \quad t \in G, \\
f(t, x) & =\phi(t)+g(x) \geqslant B^{\prime}-\nu=B \quad \text { for } x \geqslant c, \quad t \in G, \\
f(t, x) & =\phi(t)+g(x) \leqslant-B^{\prime}+\nu=-B \quad \text { for } x \leqslant-c, \quad t \in G, \\
f(t, x) & \geqslant 0 \quad \text { for } x \geqslant b, \quad f(t, x) \leqslant 0 \quad \text { for } x \leqslant-b,
\end{aligned}
$$

and the requirements of (11.vi) are satisfied provided $\nu \leqslant B^{\prime}, A<a^{-1 / 2} B \varepsilon$ for some $0<\varepsilon<$ $\gamma_{0} / 3$. Hence, we require $0<\varepsilon<\gamma_{0} / 3, \beta<a^{-1 / 2} B^{\prime} \varepsilon, \nu \leqslant B^{\prime},\left(1+a^{-1 / 2} \varepsilon\right) \nu \leqslant a^{-1 / 2} B^{\prime} \varepsilon-\beta$.

12. ANALYSIS IN THE LARGE OF THE EQUATION $u_{t r}+u_{x x x x}=f(t, x, u)$

We consider here the existence of solutions $u(t, x)$, periodic in $t$ of period $2 \pi$, of the hyperbolic problem

$$
\begin{gathered}
u_{t}+u_{x x x x}=f(t, x, u), \quad-\infty<t<+\infty, \quad 0<x<\pi, \\
u(t, 0)=u(t, \pi)=u_{x x}(t, 0)=u_{x x}(t, \pi)=0, \\
u(t+2 \pi, x)=u(t, x), \quad-\infty<t<+\infty, \quad 0<x<\pi .
\end{gathered}
$$


We initiated the discussion of this problem in [12, No. 6] for $f\left(t, x, u, u_{t}, u_{x}\right)$ depending also on $u_{t}$ and $u_{x}$. For the sake of simplicity we limit ourselves here to $f$ depending only on $t, x$, $u$. The problem had been considered by Petzeltova [20] solely in the perturbation case $f=$ $\varepsilon f_{0}, \varepsilon$ small.

Let $G=[0, \pi] \times[0,2 \pi]$, let $a=$ meas $G=2 \pi^{2}$, and let $\|u\|_{L_{2}},(u, v)_{L_{2}}$ denote the usual square norm and inner product in $G$.

Let $D$ denote the set of all functions $y(t, x)$ of class $C^{\infty}$ in $\mathbb{R} \times[0, \pi], 2 \pi$-periodic in $t$ and satisfying $y(t, 0)=y(t, \pi)$ as well as $D_{x}^{2 s} y(t, 0)=D_{x}^{2 s} y(t, \pi)=0, t \in \mathbb{R}, s=0,1,2, \ldots$ As in Section 6, let $X=A_{21}$ denote the closure of $D$ with respect to the norm $\|u\|_{X}=\left\|u_{t}\right\|_{L_{2}}+\left\|u_{x x}\right\|_{L_{2}}$, so that $X=A_{21}$ is a real Hilbert space with inner product $(u, v)_{X}=\left(u_{t}, v_{t}\right)_{L_{2}}+\left(u_{x x}, v_{x x}\right)_{L_{2}}$. The closure of $D$ with respect to the norm $\|u\|_{L_{2}}$ will be denoted by $Y$, and $Y=A_{20}=L_{2}(G)$ is identifiable with $L_{2}(G)$.

As proved in Section 6 , for $u \in X=A_{21}$, then $u$ is continuous (in $\mathbb{R} \times[0, \pi]$ ), $u_{x} \in L_{q}$ for any $1 \leqslant q<6, u_{t}, u_{x x} \in L_{2}$, and

$$
\|u\|_{\infty} \leqslant \mu_{0}\|u\|_{X}, \quad\left\|u_{x}\right\|_{L_{q}} \leqslant \mu_{q 1}\|u\|_{X}, \quad\left\|u_{i}\right\|_{L_{2}} \leqslant\|u\|_{X}, \quad\left\|u_{x x}\right\|_{L_{2}} \leqslant\|u\|_{X},
$$

for suitable absolute constants $\mu_{0}, \mu_{q 1}, 1 \leqslant q<6$, (and of course $\|u\|_{L_{q}} \leqslant \mu_{q 0}\|u\|_{X}$ for some $\mu_{q 0} \leqslant \mu_{0} a^{1 / q}, q \geqslant 1$ ).

Here, $E u=u_{t}+u_{x x x x}$, and the equation $E u=\psi$ for $\psi \in Y=L_{2}(G)$ is said to hold in the weak sense (distributions) for $u \in X=A_{21}$, provided $(u, E y)_{L_{2}}=(\psi, y)_{L_{2}}$ for all $y \in D$. This convention is justified by the fact that, if $u$ is smooth (say, $u \in A_{22}$ ) and $(u, E y)_{L_{2}}=$ $(\psi, y)_{L_{2}}$, then by integration by parts we have $(E u, y)=(\psi, y)$ for all $y \in D$, and hence $E u$ $=\psi$ a.e. in $G$.

If $f(t, x, u)$ is continuous in $\mathbb{R} \times[0, \pi] \times \mathbb{R}$ and $2 \pi$-periodic in $t$, and if $u \in X=A_{21}$ then $F(t, x)=f(t, x, u(t, x))$ is continuous in $\mathbb{R} \times[0, \pi], 2 \pi$-periodic in $t$, and there is a monotone function $\gamma_{f}(s), 0 \leqslant s<+\infty$, such that $\|F\|_{\infty} \leqslant \gamma_{f}\left(\|u\|_{X}\right)$ for any $u \in A_{21}$, where $\gamma_{f}(s)$ depends solely on $f$. Then, certainly $\|F\|_{L_{2}} \leqslant a^{1 / 2} \gamma_{f}\left(\|u\|_{X}\right)$. Alternatively, if $f(t, x, u)=\phi(t, x)+g(u)$, where $\phi$ is $2 \pi$-periodic in $t, \phi \in L_{2}(G)$, and $g: \mathbb{R} \rightarrow \mathbb{R}$ is continuous, then for $u \in X=A_{21}$, $g(u(t, x))$ is continuous in $\mathbb{R} \times[0, \pi]$ and $2 \pi$-periodic in $t,\|g(u(t, x))\|_{\infty} \leqslant \gamma_{g}\left(\|u\|_{X}\right)$ and $\|F\|_{L_{2}} \leqslant\|\phi\|_{L_{2}}+a^{1 / 2} \gamma_{g}\left(\|u\|_{X}\right)$, where the monotone function $\gamma_{g}(s), 0 \leqslant s<+\infty$, depends only on $g$. We shall denote by $\gamma(s)$ any function such that $\|N u\|_{L_{2}}=\|f(t, x, u(t, x))\|_{L_{2}} \leqslant \gamma(R)$ whenever $u \in X$ and $\|u\|_{X} \leqslant R$.

Let $e_{k l}(t, x), k=0, \pm 1, \pm 2, \ldots, l=1,2, \ldots$, denote all elements of the form $\pi^{-1} \sin l x$, $2^{1 / 2} \pi^{-1} \cos k t \sin l x, 2^{1 / 2} \pi^{-1} \sin k t \sin l x$. These elements $e_{k l}$ are orthonormal in $Y$, and any $u$ $\in Y=A_{20}=L_{2}(G)$ has Fourier series $u(t, x)=\Sigma b_{k l} e_{k l}$ with $\Sigma b_{k l}^{2}<+\infty, b_{k l}=\left(u, e_{k l}\right)_{Y}=$ $\left(u, e_{k l}\right)_{L_{2}}$.

Now let $E_{k l}(t, x), k=0, \pm 1, \pm 2, \ldots, l=1,2, \ldots$ denote all elements of the form $\pi^{-1} l^{-2} \sin l x, 2^{1 / 2} \pi^{-1}\left(k^{2}+l^{4}\right)^{-1 / 2} \cos k t \sin l x, 2^{1 / 2} \pi^{-1}\left(k^{2}+l^{4}\right)^{-1 / 2} \sin k t \sin l x$. These elements are orthonormal in $X$, and any $u \in X=A_{21}$ has Fourier series in $X$ of the form $u(t, x)=\Sigma a_{k l} E_{k l}$, $a_{k l}=\left(u, E_{k l}\right)_{X}$, and $u_{t}-u_{x x}$ have Fourier series $u_{t}=\Sigma\left(a_{k l} k\right) E_{k l}^{\prime}, u_{x x}=\Sigma\left(a_{k l} l^{2}\right) E_{k}^{\prime \prime}$, where $E_{k l}^{\prime}$ is obtained from $E_{k l}$ by changing $\cos k t, \sin k t$ into $\sin k t, \cos k t$ respectively, and $E_{k l}^{\prime \prime}$ is obtained from $E_{k l}$ by changing $\sin l x$ into $-\sin l x$. On the other hand, if $b_{k l}=\left(u, e_{k l}\right)_{L_{2}}$, then

$$
\begin{aligned}
& a_{k l}=\left(u, E_{k l}\right)_{X}=\left(k^{2}+l^{4}\right)^{1 / 2}\left(u, e_{k l}\right)_{Y}=\left(k^{2}+l^{4}\right)^{1 / 2} b_{k l}, \\
& a_{k l} E_{k l}=\left(u, E_{k l}\right) E_{k l}=\left(k^{2}+l^{4}\right)^{1 / 2}\left(u, e_{k l}\right) \cdot\left(k^{2}+l^{4}\right)^{-1 / 2} e_{k l}=b_{k l} e_{k l} .
\end{aligned}
$$

For $u \in X$, then $u, u_{t}, u_{x x}$ have Fourier series in $Y=L_{2}, u=\Sigma b_{k l} e_{k l}, u_{t}=\Sigma b_{k l} k e_{k l}^{\prime}, u_{x x}=$ 
$\Sigma b_{k l} l^{2} e_{k l}^{\prime \prime}$, where $e_{k l}^{\prime}$ is obtained from $e_{k l}$ by changing $\cos k t, \sin k t$ into $\sin k t, \cos k t$, and $e_{k l}^{\prime \prime}$ is obtained from $e_{k l}$ by changing $\sin l x$ into $-\sin l x$. Thus, $\Sigma b_{k l}^{2}\left(k^{2}+l^{4}\right)<+\infty$.

Let $X_{0}$ denote the subspace of $X$ generated by the elements $e_{k l}$ with $k^{2}=l^{4}$. Then, for $u^{*} \in X_{0}, u^{*}=\Sigma_{k^{2}=} b_{k l} e_{k l}$ where $\Sigma$ ranges over all $k=0, \pm 1, \pm 2, \ldots, l=1,2, \ldots$, with $k^{2}=l^{4}$. By distribution theory $X_{0}$ is the subspace of $X$ of all weak solutions of $E u=0$, or $\left(u, y_{t t}+y_{x x x x}\right)=0$ for all $y \in D$, that is, $X_{0}=$ ker $E$.

Note that, for $u^{*} \in X_{0}$, or $\left(u^{*}, y_{t t}+y_{x x x x}\right)_{L_{2}}=0$ for all $y \in D$, we also have, by integration by parts, $-\left(u_{t}^{*}, y_{t}\right)_{L_{2}}+\left(u_{x x}^{*}, y_{x x}\right)_{L_{2}}=0$ for all $y \in D$. If $v \in X_{0}$ and we approximate $v$ by elements $y \in D$ in such a way that $y_{t}, y_{x x}$ approximate $v_{t}^{*}, v_{x x}^{*}$ in $L_{2}$, we also have $-\left(u_{t}^{*}, v_{t}^{*}\right)_{L_{2}}+\left(u_{x x}^{*}, v_{x x}^{*}\right)_{L_{2}}=0$. Thus,

$$
\left(u_{t}^{*}, v_{t}^{*}\right)_{L_{2}}=\left(u_{x x}^{*}, v_{x x}^{*}\right)_{L_{2}}, \quad\left\|u_{t}^{*}\right\|_{L_{2}}=\left\|u_{x x}^{*}\right\|=2^{-1}\left\|u^{*}\right\|_{X} \quad \text { for all } u^{*}, v^{*} \in X_{0} .
$$

Let $P$ denote the natural projection of $X$ onto $X_{0}$. For $u \in X_{1}=(I-P) X$, then $u=$ $\Sigma b_{k l} e_{k l}$ with $\Sigma b_{k l}\left(k^{2}+l^{4}\right)<+\infty$, where $\Sigma$ ranges over all $k=0, \pm 1, \ldots, l=1,2 \ldots$, with $k^{2} \neq l^{4}$. Let $Y_{0}, Y_{1}$ denote the analogous decomposition of $Y$, and let $Q$ be the natural projection of $Y$ onto $Y_{0}$. We can now define the operator $H: Y_{1} \rightarrow X_{1}$. For $u \in Y_{1}$, or $u=\Sigma_{k^{2} \neq \mu} b_{k l} e_{k l}$ $\Sigma b_{k l}^{2}<+\infty$, let us take $v=H u=\Sigma_{k^{2} \neq \mu_{k l}} b_{k l}\left(-k^{2}+l^{4}\right)^{-1} e_{k l}$. As we have seen in Section 6, $v=$ $H u \in X_{1}=(I-P) X$, thus $v \in A_{21}$, and $v$ is a weak solution of $E v=u$. Moreover, $\|H u\|_{L_{2}} \leqslant\|u\|_{L_{2}},\|H u\|_{X} \leqslant\|u\|_{L_{2}}$ for $u \in Y_{1}$, and $\|H\|$ is a linear bounded operator from $Y_{1}$ onto $X_{1}$ with $\|H\|=L=1$. With $X, Y, P, Q, H$ as above, axioms (a), (b), (c) of Section 3 are certainly satisfied.

We now define the finite dimensional subspaces $X_{0 n}$ of $X_{0}$ as follows: $X_{0 n}$ is the subspace of $X_{0}$ in $X$ generated by all $e_{k l}$ with $k^{2}=l^{4}, l=1, \ldots, \mathrm{n}$.

Let $X_{1 n}$ be the subspace of $X$ generated by all $e_{k l}$ with $k^{2} \neq l^{4}, k=0, \pm 1, \ldots, \pm n, l=$ $1, \ldots, n$. Let $R_{n}, S_{n}$ be the appropriate orthogonal projections $R_{n}: X_{1} \rightarrow X_{1 n}, S_{n}: X_{0} \rightarrow X_{0 n}$. Let $Y_{0 n}$ be the subspace of $Y_{0}$ in $Y$ generated by $e_{k l}, k^{2}=l^{4}, l=1, \ldots, n$. Thus $S_{n}^{\prime}: Y_{0} \rightarrow Y_{0 n}$ can be defined as the orthogonal projection of $Y_{0}$ onto $Y_{0 n}$ in $Y$.

Finally, let $\alpha_{n}: Y_{0 n} \rightarrow X_{0 n}$ denote the map defined by

$$
\alpha_{n} y=\Sigma_{0 n} b_{k l} e_{k l}, \quad \text { where } y=\Sigma_{0 n} b_{k l} e_{k l}, \quad b_{k l}=\left(y, e_{k l}\right)_{L_{2}},
$$

where $\Sigma_{0 n}$ ranges over all $k=0, \pm 1, \ldots, \pm n, l=1,2, \ldots, n$, with $k^{2}=l^{4}$, (hence $|k| \geqslant 1$ ). Clearly $\alpha_{n}^{-1}(0)=0$ that is, $\alpha_{n} S_{n}^{\prime} Q N u=0$ is equivalent to $S_{n}^{\prime} Q N u=0$, and moreover $S_{n}^{\prime} Q N u=0$ if and only if $\left(S_{n}^{\prime} Q N u, v^{*}\right)=0$ for all $v^{*} \in X_{0 n}$.

However, we note that here, for $y \in Y_{0 n}, \alpha_{n} y=y \in X_{0 n}$, but $\alpha_{n} y$ in $X$ has a norm in $X$ which is quite different from the norm of $y$ in $Y=L_{2}$, namely

$$
\left\|\alpha_{n} y\right\|_{X}=\|y\|_{X}=\left(\sum_{0 n} b_{k l}^{2}\left(k^{2}+l^{4}\right)\right)^{1 / 2}, \quad\|y\|_{Y}=\left(\sum_{0 n} b_{k l}^{2}\right)^{1 / 2} .
$$

We may note that, for any element $x \in X_{1}$, then $x$ is a bounded function on $G$, and $R_{n} x$ is also a bounded function on $G,\left\|R_{n} x\right\|_{L_{2}} \leqslant\|x\|_{L_{2}}$, but $\left\|R_{n} x\right\|_{\infty}$ may by much larger than $\|x\|_{\infty}$, a well known phenomenon in Fourier series. Analogously, if $y \in Y_{0}$ and $y$ happens to be bounded in $G$, then $S_{n}^{\prime} y \in Y_{0}$ is also bounded in $G,\left\|S_{n}^{\prime} y\right\|_{L_{2}} \leqslant\|y\|_{L_{2}}$, but the norm $\left\|S_{n}^{\prime} y\right\|_{\infty}$ may be much larger then $\|y\|_{\infty}$.

Finally, let $J_{n}: X_{0 n} \rightarrow X_{0 n}$ be the linear operator defined as follows. Let $\alpha, \beta \geqslant 0$ be constants with $\alpha+\beta>0$, for the moment arbitrary. For any $u \in X_{0}$ we have $u=\Sigma b_{k l} e_{k l}$, where $\Sigma$ ranges over all $k, l$ with $k^{2}=l^{4}, k=0, \pm 1, \pm 2, \ldots, l=1,2, \ldots, n$, (hence $|k| \geqslant 1$ ). We 
take $J u=\Sigma k{ }^{1} b_{k l} e_{k l}^{*}$, where $e_{k l}^{*}$ is obtained from $e_{k l}$ by replacing $\cos k t$ by $\sin k t$, and $\sin k t$ by $-\cos k t$. Then, $J$ is a isomorphism, and further $(J u)_{t}=u$. Let us take in (5.3), (5.4), $J_{n}=$ $-\alpha J^{2}+\beta I$, where $I$ is the identity operator. Then, for $u \in X_{0}, u=\Sigma b_{k l} e_{k l}$, the sum ranging over all $k, l$ with $k^{2}=l^{4}$, and $J_{n} u=\left(\alpha k^{-2}+\beta\right) b_{k l} e_{k l}$, and $J_{n}$ is a isomorphism.

The original equations (12.1-12.3), are now written in the abstract form $E x=N x$, or in the equivalent form of auxiliary and bifurcation equations

$$
u_{1}=H(I-Q) N u, \quad Q N u=0, \quad u=u^{*}+u_{1} \in X .
$$

For every integer $n$, we have then the reduced equations

$$
u_{1}=R_{n} H(I-Q) N u, \quad S_{n}^{\prime} Q N u=0, \quad u=u^{*}+u_{1} \in X_{n},
$$

and, for each $n$, we apply Theorem (4.i) (actually, Lemma (3.i), or statement (2.i)), to the reduced equations

$$
u_{1}=R_{n} H(I-Q) N u, \quad J_{n} \alpha_{n} S_{n}^{\prime} Q N u=0, \quad u=u^{*}+u_{1} \in X_{n}=X_{n 0} \times X_{n \mathbf{1}} .
$$

Below, we shall show that there are numbers $R_{0}, r>0$ such that, for every integer $n$, there is at least one solution $u_{n} \in X_{n}, u_{n}=u_{n}^{*}+u_{n 1}, u_{n}^{*} \in X_{0 n}, u_{n 1} \in X_{1 n}$, hence, $u_{n} \in X$, $u_{n}^{*} \in X_{0}, u_{n 1}^{*} \in X_{1}, n=1,2, \ldots$, with $\left\|u_{n}^{*}\right\|_{X} \leqslant R_{0}\left\|u_{n 1}\right\|_{X} \leqslant r,\left\|u_{n}\right\| \leqslant\left(R_{0}^{2}+r^{2}\right)^{1 / 2}$ for all $n$.

Proceeding as in Section 5, we now introduce the space $\mathscr{X}$. For $\mathscr{X}$ we choose $C$, the space of continuous functions on $\mathbb{R} \times[0, \pi], 2 \pi$-periodic in $t$. Then $\left\|u_{n}\right\|_{X}$ is bounded, and the sequence $u_{n}(t, x),(t, x) \in \mathbb{R} \times[0, \pi]$, is equicontinuous. By applying Ascoli's theorem we see that any weak limit element of $\left[u_{n}\right]$ in $X$ is a strong limit in $\mathscr{X}$. Moreover, as we have seen in the Remark at the end of Section 5 , there is a subsequence, say still $[n]$ such that $u_{n} \rightarrow u$ uniformly to a continuous function $u$. Then the functions $N u_{n}$ are equibounded and converge pointwise and uniformly to the bounded functions $N u$ as $n \rightarrow \infty$. Proceeding to the limit in the coupled system of equations (12.6), we obtain that $u \in \mathscr{X}$ is a solution in the weak sense of the original problem.

Here the solution $u$ is continuous with given modulus of continuity, $u_{t}, u_{x}, u_{x x}$ exist in $L_{2}$, $u_{x x x x}$ exist in the distributional sense, and they satisfy the original equation in the weak sense.

(12.i) Let $f(t, x, s)$ be of class $C^{1}$ in $\mathbb{R} \times[0, \pi] \times \mathbb{R}$, and let $\gamma(s)$ be the function defined above. Let us assume that there are constants $R_{0}, r$ such that $L \gamma(R) \leqslant r$ with $R=$ $\left(R_{0}^{2}+r^{2}\right)^{1 / 2}$, and such that, for all $u^{*} \in X_{0}, u_{1} \in X_{1},\left\|u^{*}\right\|_{X}=R_{0},\left\|u_{1}\right\|_{X} \leqslant r, X=A_{21}$,

$$
\left.\alpha \int_{G} f(t, x, u) u^{*} \mathrm{~d} t \mathrm{~d} x+\beta \int_{G}(f(t, x, u))_{r} u_{t}^{*} \mathrm{~d} t \mathrm{~d} x \geqslant 0 \quad \text { [or } \leqslant 0\right] .
$$

Then the hyperbolic problem (12.1-12.3) has at least one solution $u(t, x) \in X=A_{21}$ with $\|u\|_{X} \leqslant R$.

Proof. We shall only show that (5.i) applies. Thus, we have to verify hypotheses (a) and (b) of (5.i). Actually, (a) is satisfied, and, by Remark 1 of Section 5, it is enough to verify that

$$
\left.\left(J_{n} \alpha_{n} S_{n}^{\prime} Q N u, u^{*}\right)_{X} \geqslant 0 \quad \text { [or } \leqslant 0\right]
$$

for all $u^{*} \in X_{0 n},\left\|u^{*}\right\|_{X}=R_{0}, u=u^{*}+u_{1}, u_{1} \in X_{1 n},\left\|u_{1}\right\| \leqslant r$. Note that, because of the choice of $J_{n}$, by integration by parts we have 


$$
\begin{aligned}
\left(J_{n} \alpha_{n} S_{n}^{\prime} Q N u, u^{*}\right)_{X} & =2\left(\left(J_{n} \alpha_{n} S_{n}^{\prime} Q N u\right)_{t}, u_{t}^{*}\right)_{L_{2}} \\
& =-2 \alpha\left(\left(J^{2} \alpha_{n} S_{n}^{\prime} Q N u\right)_{t}, u_{t}^{*}\right)_{L_{2}}+2 \beta\left(\left(\alpha_{n} S_{n}^{\prime} Q N u\right)_{n}, u_{t}^{*}\right)_{L_{2}} \\
& =-2 \alpha\left(J \alpha_{n} S_{n}^{\prime} Q N u, u_{t}^{*}\right)_{L_{2}}+2 \beta\left(\left(\alpha_{n} S_{n}^{\prime} Q N u\right)_{t}, u_{t}^{*}\right)_{L_{2}} \\
& =2 a\left(\alpha_{n} S_{n}^{\prime} Q N u, u^{*}\right)_{L_{2}}+2 \beta\left(\left(\alpha_{n} S_{n}^{\prime} Q N u\right)_{t}, u_{t}^{*}\right)_{L_{z}}
\end{aligned}
$$

Now we have

$$
\begin{aligned}
N u & =\Sigma\left(N u, e_{k l}\right)_{L_{2}} e_{k l}, \\
Q N u & =\sum_{k^{2}=I^{4}}\left(N u, e_{k l}\right)_{L_{2}} e_{k l}, \\
\alpha_{n} S_{n}^{\prime} Q N u & =\Sigma^{*}\left(N u, e_{k l}\right)_{L_{2}} e_{k l},
\end{aligned}
$$

where $\Sigma^{*}$ ranges over all $k, l$ with $k^{2}=l^{4}, l=1, \ldots, n$. Thus,

$$
\begin{aligned}
\left(J_{n} \alpha_{n} S_{n}^{\prime} Q N u, u^{*}\right)_{X} & =2 \alpha\left(\alpha_{n} S_{n}^{\prime} Q N u, u^{*}\right)_{L_{2}}+2 \beta\left(\alpha_{n} S_{n}^{\prime} Q(N u)_{t}, u_{f}^{*}\right)_{L_{2}} \\
& =2 \alpha \int_{G} f(t, x, u) u^{*} \mathrm{~d} t \mathrm{~d} x+2 \beta \int_{G}(f(t, x, u))_{t} u_{t}^{*} \mathrm{~d} t \mathrm{~d} x,
\end{aligned}
$$

where $(f(t, x, u))_{t}=f_{t}+f_{u} u_{t}$.

$A$ set of inequalities implying relation (12.7)

Note that, for $x=A_{21}$, the elements $u \in X$ with $\|u\|_{X}=1$ are functions $u(t, x)$ in $G$ with a common modulus of continuity $\omega_{0}(\zeta), 0 \leqslant \zeta<+\infty$. Let $a=$ meas $G=2 \pi^{2}$, and let $\mu_{1}, \mu_{q 1}$, $1 \leqslant q<6$, and $\mu_{q 0} \leqslant \mu_{0} a^{1 / q}, 1 \leqslant q<\infty$, be the constants for which relations (12.4) hold.

Let $R_{0}, r, \Gamma, \eta$ be positive numbers which we shall determine later.

If $v^{*} \in X_{0},\left\|v^{*}\right\|_{X}=1, \sigma \in X_{1},\|\sigma\|_{X} \leqslant r$ are given elements, then for $0 \leqslant \rho<R_{0}, \rho v^{*}$ has modulus of continuity $R_{0} \omega_{0}(\zeta)$, and $\|\sigma\|_{x} \leqslant \mu_{0} r$. Let $G^{\prime}=\left[(t, x) \in G|| v^{*}(t, x) \mid \leqslant \Gamma\right]$, $G^{\prime \prime}=G-G^{\prime}$.

Let $k(s), 0 \leqslant s \leqslant D_{0}=\operatorname{diam} \bar{G}$, denote the function defined in $(9 . i)$, so that $k(s)>0$ for $0<s \leqslant D_{0}, k(0)=0$, and for every point $P \in \bar{G}$ and $\left.U(P, s)=\left|Q \in \mathbb{R}^{2}\right||Q-P| \leqslant s\right]$, we also have meas $[U(P, s) \cap G] \geqslant k(s), 0<s \leqslant D_{0}$. We have seen that it is not restrictive to assume $k=k(s)$ continuous in $[0, D]$. Actually, because of the periodicity, in the present situation, we can take $k=k(s)=2^{-1} \pi s^{2}$, with inverse function $s=s(k)=\left(2 \pi^{-1} k\right)^{1 / 2}$, and meas $[U(P, s) \cap G] \geqslant 2^{-1} \pi s^{2}$ for all $s \geqslant 0$.

(12.ii) Let $f(t, x, u)=\phi(t, x)+g(u)$, where $\phi$ is of class $C^{1}$ and $2 \pi$-periodic in $t$, and $g: \mathbb{R}$ $\rightarrow \mathbb{R}$ is of class $C^{1}$. Let us assume that:

$$
\begin{aligned}
& u g(u) \geqslant 0, \quad|g(u)| \leqslant C, \quad\left|g_{u}(u)\right| \leqslant D, \quad-d^{\prime} \leqslant g_{u}(u) \leqslant d^{\prime \prime}, \quad u \in \mathbb{R}, \\
& g(u) \geqslant B \quad \text { for } u \geqslant b, \quad g(u) \leqslant-B \quad \text { for } u \leqslant-b, \\
& g_{u}(u) \geqslant d>0 \quad \text { for }|u| \leqslant \delta,
\end{aligned}
$$

for suitable positive constants $B, C, d, d^{\prime}, d^{\prime \prime}, \delta$ with $b<\delta, B<C, D=\max \left[d^{\prime}, d^{\prime \prime}\right]$. 
Let $\Gamma, \eta, r, R_{0}, \alpha, \beta$ be positive constants such that

$$
\begin{aligned}
& 0<\eta<a=2 \pi^{2}, \quad R_{0} \Gamma \geqslant \mu_{0} r+b, \quad R_{0} \Gamma+R_{0} \omega_{0}(\sqrt{2 / \pi})+\mu_{0} r \leqslant \delta, \\
& M_{1}=\alpha B R_{0} \Gamma \eta-\alpha C \mu_{10} r-\beta d^{\prime \prime}\left(2^{-1} R_{0}^{2}+r^{2}\right)-\beta D\left(2^{-1} R_{0}^{2}+r^{2}\right)^{1 / 2} r>0, \\
& M_{2}=\beta d\left(2^{-1} R_{0}^{2}\right)-\beta D\left(2^{-1} R_{0}^{2}+r^{2}\right)^{1 / 2} r-\alpha C R_{0} \mu_{10}>0, \\
& R_{0}\left(\alpha \mu_{20}\|\phi\|_{L_{2}}+\beta 2^{-1 / 2}\left\|\phi_{\|}\right\|_{L_{2}}\right) \leqslant \min \left[M_{1}, M_{2}\right] .
\end{aligned}
$$

Then, for $\rho=R_{0}, v^{*} \in X_{0},\left\|v^{*}\right\|_{X}=1, \sigma \in X_{1},\|o\|_{X} \leqslant r$, we have

$$
\begin{gathered}
\alpha \int_{G}\left[\phi(t, x)+g\left(\rho v^{*}+\sigma\right)\right]\left(\rho v^{*}\right) \mathrm{d} t \mathrm{~d} x+\beta \int_{G}\left[\phi_{\lambda}(t, x)+\left(g \left(\rho v^{*}\right.\right.\right. \\
\left.+\sigma))_{t}\right]\left(\rho v_{t}^{*}\right) \mathrm{d} t \mathrm{~d} x \geqslant 0 .
\end{gathered}
$$

If, in addition,

$$
\|\phi\|_{L_{2}}+2^{1 / 2} \pi C \leqslant r
$$

then problem (12.1-12.3) with $f=\phi+g(u)$ has at least one solution $u \in X=A_{21}$, $u=u^{*}+u_{1}, u^{*} \in X_{0}, u_{1} \in X_{1},\left\|u^{*}\right\|_{X} \leqslant R_{0},\left\|u_{1}\right\|_{X} \leqslant r,\|u\|_{X} \leqslant R=\left(R_{0}^{2}+r^{2}\right)^{1 / 2}$.

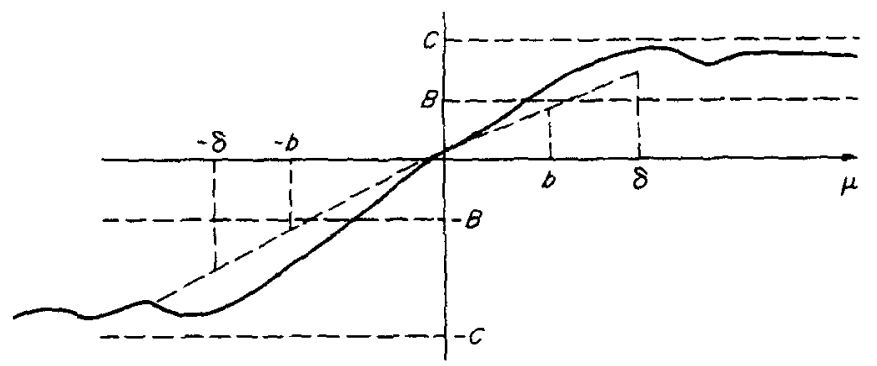

Proof. Note that, for $u \in X=A_{21},\|u\|_{X} \leqslant R, F(t, x)=f(t, x, u(t, x))=\phi(t, x)+\mathrm{g}(u(t$, $x)$ ) we certainly have $\|F\|_{Y}=\|F\|_{L_{2}} \leqslant\|\phi\|_{L_{2}}+C(\text { meas } G)^{1 / 2}=\|\phi\|_{L_{2}}+2^{1 / 2} \pi C$, and we can take $\gamma(s)=\|\phi\|_{L_{2}}+2^{1 / 2} \pi C$, a constant function. Analogously, we have $\|F\|_{\infty} \leqslant \mu_{0}\|F\|_{L_{2}} \leqslant \mu_{0}\left(\|\phi\|_{L_{2}}+\right.$ $\left.2^{1 / 2} \pi C\right)$, and we take $\gamma_{f}(s)=\mu_{0}\left(\|\phi\|_{L_{2}}+2^{1 / 2} \pi C\right)$, also a constant function. Finally, since $L=$ $\|H\|=1$, requirement $L \gamma(R) \leqslant r$ of $(12 . i)$ reduces to the inequality (12.9).

Note that, for $v^{*} \in X_{0},\left\|v^{*}\right\|_{X}=1$, we have, from (12.5), $\left\|v_{i}^{*}\right\|_{L_{2}}^{2}=\left\|v_{x x}^{*}\right\|_{L_{2}}^{2}=2^{-1}\left\|v^{*}\right\|_{X}^{2}$ and $\left\|v_{t}^{*}\right\|_{L_{2}}=\left\|v_{x x}^{*}\right\|_{L_{2}}=2^{-1 / 2}$. For $u=\rho v_{t}^{*}, v=\sigma_{t}, \rho=R_{0}, v^{*} \in X_{0}, \sigma \in X_{1},\left\|v^{*}\right\|_{X}=1,\|\sigma\|_{X} \leqslant r$, not only $v^{*}$ and $\sigma$ are orthogonal in $L_{2}$, but also $v^{*}$, and $\sigma_{l}$. Hence

$$
R_{0}^{2} 2^{-1} \leqslant\left\|\rho v_{t}^{*}+\sigma_{t}\right\|_{L_{2}}^{2}=\left\|\rho v_{t}^{*}\right\|_{L_{2}}^{2}+\left\|\sigma_{t}\right\|_{L_{2}}^{2} \leqslant R_{0}^{2} 2^{-1}+r^{2} .
$$

As stated, let $\left.G^{\prime}=\left[(t, x) \in G|| v^{*}(t, x)\right\} \leqslant \Gamma\right], G^{\prime \prime}=G-G^{\prime}$.

First, let us assume that meas $G^{\prime \prime}<\eta$. For $P \in \bar{G}$ we have meas $(U(P, s) \cap G)>k(s)$ and thus for $s=s(\eta)$ we also have meas $(U(P, s) \cap G)>k(s)=\eta$, that is, the ball $U(P, s)$ is not filled by points of $G^{\prime \prime}$, or $U(P, s) \cap G^{\prime} \neq \varnothing$. In other words, any points $P \in \bar{G}$ is at a distance $\leqslant s=s(\eta)$ from points $Q$ of $G^{\prime}$. Hence, $\left|\rho v^{*}(P)-\rho v^{*}(Q)\right| \leqslant R_{0} \omega_{0}(s)$ with $v^{*}(Q) \leqslant \Gamma$, and finally

$$
\left|\rho v^{*}(P)\right| \leqslant R_{0} \Gamma+R_{0} \omega_{0}(s(\eta)) \text { for all } P \in \bar{G}
$$


Now $s(\eta)=2^{1 / 2} \pi^{-1 / 2} \eta^{1 / 2}$ and the third relation (12.8) becomes

$$
R_{0} \Gamma+R_{0} \omega_{0}(s(\eta))+\mu_{0} r \leqslant \delta
$$

and, for $\rho=R_{0}$ and meas $G^{\prime \prime}<\eta$, we have

$$
\left|\rho v^{*}+\sigma\right| \leqslant R_{0} \Gamma+R_{0} \omega_{0}(s(\eta))+\mu_{0} r \leqslant \delta .
$$

Hence, $g_{u} \geqslant d,|g| \leqslant C$, and

$$
\begin{aligned}
\int_{G} g_{u}\left(\rho v^{*}+\sigma\right)\left(\rho v_{t}^{*}+\sigma_{t}\right)\left(\rho v_{t}^{*}\right) \mathrm{d} t \mathrm{~d} x & =\int_{G} g_{u}\left(\rho v^{*}+\sigma\right)\left(\rho v_{t}^{*}+\sigma_{t}\right)^{2} \mathrm{~d} t \mathrm{~d} x \\
& -\int_{G} g_{u}\left(\rho v^{*}+\sigma\right)\left(\rho v_{t}^{*}+\sigma_{t}\right) \sigma_{t} \mathrm{~d} t \mathrm{~d} x \\
& \geqslant d\left\|\rho v_{t}^{*}+\sigma_{t}\right\|_{L_{2}}^{2}-D\left\|\rho v_{t}^{*}+\sigma_{t}\right\|_{L_{2}}\left\|\sigma_{t}\right\|_{L_{2}} \\
& \geqslant d\left(2^{-1} R_{0}^{2}\right)-D\left(2^{-1} R_{0}^{2}+r^{2}\right)^{1 / 2} r \\
\int_{G} g\left(\rho v^{*}+\sigma\right)\left(\rho v^{*}\right) \mathrm{d} t \mathrm{~d} x & \geqslant-C R_{0}\left\|v^{*}\right\|_{L_{1}} \geqslant-C R_{0} \mu_{10}
\end{aligned}
$$

Let us assume now that meas $G^{\prime \prime} \geqslant \eta$. Then, for $\rho=R_{0}, t \in G^{\prime \prime}$, we have $\left|v^{*}\right| \geqslant \Gamma$,

$$
\begin{aligned}
& \rho v^{*}+\sigma \geqslant R_{0} \Gamma-\mu_{0} r \geqslant b \quad \text { if } v^{*} \geqslant \Gamma, \\
& \rho v^{*}+\sigma \leqslant-R_{0} \Gamma+\mu_{0} r \leqslant-b \quad \text { if } v^{*} \leqslant-\Gamma,
\end{aligned}
$$

and in any case $g\left(\rho v^{*}+\sigma\right)\left(\rho v^{*}\right) \geqslant B R_{0} \Gamma$. Then

$$
\begin{aligned}
& \int_{G^{*}} g\left(\rho v^{*}+\sigma\right)\left(\rho \sigma^{*}\right) \mathrm{d} t \mathrm{~d} x \geqslant B R_{0} \Gamma \eta \\
& \int_{G^{\prime}} g\left(\rho v^{*}+\sigma\right)\left(\rho v^{*}\right) \mathrm{d} t \mathrm{~d} x=\int_{G^{\prime}} g\left(\rho v^{*}+\sigma\right)\left(\rho v^{*}+\sigma\right) \mathrm{d} t \mathrm{~d} x-\int_{G^{\prime}} g\left(\rho v^{*}+\sigma\right) \sigma \mathrm{d} t \mathrm{~d} x \\
& \geqslant 0-C\|\sigma\|_{L_{1}} \geqslant-C \mu_{10} r \\
& \int_{G} g_{u}\left(\rho v^{*}+\sigma\right)\left(\rho v_{t}^{*}+\sigma_{t}\right)\left(\rho v_{t}^{*}\right) \mathrm{d} t \mathrm{~d} x=\int_{G} g_{u}\left(\rho v^{*}+\sigma\right)\left(\rho v_{t}^{*}+\sigma_{t}\right)^{2} \mathrm{~d} t \mathrm{~d} x \\
&-\int_{G} g_{u}\left(\rho v^{*}+\sigma\right)\left(\rho v_{t}^{*}+\sigma_{t}\right) \sigma_{t} \mathrm{~d} t \mathrm{~d} x \\
& \geqslant-d^{\prime}\left\|\rho v_{t}^{*}+\sigma_{t}\right\|_{L_{2}}^{2}-D\left\|\rho v_{t}^{*}+\sigma_{t}\right\|_{L_{2}}\left\|\sigma_{t}\right\|_{L_{2}} \\
& \geqslant-d^{\prime}\left(2^{-1} R_{0}^{2}+r^{2}\right)-D\left(2^{-1} R_{0}^{2}+r^{2}\right)^{1 / 2} r .
\end{aligned}
$$

We have now in any case

$$
\begin{aligned}
& \alpha \int_{G} g\left(\rho v^{*}+\sigma\right)\left(\rho v^{*}\right) \mathrm{d} t \mathrm{~d} x+\beta \int_{G} g_{u}\left(\rho v^{*}+\sigma\right)\left(\rho v_{t}^{*}+\sigma_{t}\right)\left(\rho v_{t}^{*}\right) \mathrm{d} t \mathrm{~d} x \\
& \quad \geqslant \min \left\{\beta d\left(2^{-1} R_{0}^{2}\right)-\beta D\left(2^{-1} R_{0}^{2}+r^{2}\right)^{1 / 2} r-\alpha \lambda R_{0} \mu_{10} ;\right. \\
& \left.\alpha B R_{0} \Gamma \eta-\alpha C \mu_{10} r-\beta d^{\prime}\left(2^{-1} R_{0}^{2}+r^{2}\right)-\beta D\left(2^{-1} R_{0}^{2}+r^{2}\right)^{1 / 2} r\right\} .
\end{aligned}
$$


Finally, for $f=\phi+g$, relation (12.7) is satisfied, since by (12.8), we have

$$
\begin{aligned}
& \left.\alpha \int_{G} f\left(t, x, \rho v^{*}+\sigma\right)\left(\rho v^{*}\right) \mathrm{d} t \mathrm{~d} x+\beta \int_{G}\left(f\left(t, x, \rho v^{*}+\sigma\right)\right)\right)_{t}\left(\rho v_{t}^{*}\right) \mathrm{d} t \mathrm{~d} x \\
& \quad=\alpha \int_{G}\left[\phi+g\left(\rho v^{*}+\sigma\right)\right]\left(\rho v^{*}\right) \mathrm{d} t \mathrm{~d} x+\beta \int_{G}\left[\phi_{t}+g_{t}\left(\rho v^{*}+\sigma\right)\left(\rho v_{t}^{*}+\sigma_{t}\right)\right]\left(\rho v_{t}^{*}\right) \mathrm{d} t \mathrm{~d} x \\
& \quad \geqslant \min \left[M_{1}, M_{2}\right]+\alpha \int_{G} \phi \rho v^{*} \mathrm{~d} t \mathrm{~d} x+\beta \int_{G} \phi_{t} \rho v_{t}^{*} \mathrm{~d} t \mathrm{~d} x \\
& \quad \geqslant \min \left[M_{1}, M_{2}\right]-R_{0}\left(\alpha \mu_{20}\|\phi\|_{L_{2}}-\beta 2^{-1 / 2}\|\phi\|_{L_{2}}\right) \geqslant 0 .
\end{aligned}
$$

This concludes the proof of (12.ii).

(12.iii) Let $f(t, x, u)=\phi(t, x)+g(u)$, where $\phi$ is of class $C^{1}$ and $2 \pi$-periodic in $t$, and $g: \mathbb{R} \rightarrow \mathbb{R}$ is of class $C^{1}$. Let us assume that

$$
\begin{aligned}
& u g(u) \geqslant 0, \quad|g(u)| \leqslant C \quad \text { for all }|u| \leqslant S, \\
& g(u) \geqslant B \quad \text { for } u \geqslant b, \quad g(u) \leqslant-B \quad \text { for } u \leqslant-b, \\
& -d \leqslant g_{u}(u) \leqslant d^{\prime \prime} \quad \text { for all }|u| \leqslant S, \quad D=\max \left(d^{\prime}, d^{\prime \prime}\right), \\
& g_{u}(u) \geqslant d \quad \text { for all }|u| \leqslant \delta,
\end{aligned}
$$

for some positive constants $B, C, b, \delta, S, d, B<C, b<\delta<S$. We shall also assume that, for given numbers $0<\varepsilon<1, \theta>0$ we have $B=(1-\varepsilon) d b, C=(1+\theta) d \delta$, and that $d^{\prime}$ $=k^{\prime} d, d^{\prime \prime}=k^{\prime \prime} d, D=k d, k=\max \left(k^{\prime}, k^{\prime \prime}\right)$.

Then, for any given $b, \delta, \varepsilon, \theta, k, k^{\prime \prime}=k$, there are numbers $d_{0}, k_{o}^{\prime}, A_{0}, B_{0}$ such that for $d$ $\leqslant d_{0}, k^{\prime} \leqslant k_{0}^{\prime}$, and all $\phi \in C^{1}$ with

$$
\|\phi\|_{L_{2}}+A_{0}\left\|\phi_{t_{2}}\right\|_{L_{2}} \leqslant B_{0}
$$

problem (12.1-12.3) has at lcast a solution $u \in A_{21}$ with $\|u\|_{X} \leqslant R$, where $R$ depends only on the constants above, and then $S=\mu_{0} R$.

Proof. We shall apply statement (12.ii). First we rewrite inequalities (12.8-12.9) in a slightly stronger form

$$
\begin{aligned}
& 0<\eta<2 \pi^{2}, \quad R_{0} \Gamma \geqslant \mu_{0} r+h, \quad R_{0} \Gamma+\omega_{0}(\sqrt{2 \eta / \pi})+\mu_{0} r \leqslant \delta, \\
& (b d)\left(R_{0} \Gamma\right) \eta>(1+\theta)(\delta d) \mu_{10} r+(\beta / \alpha) d^{\prime}\left(2^{-1} R_{0}^{2}+r^{2}\right)+(\beta / \alpha) D\left(2^{-1} R_{0}^{2}+r^{2}\right)^{1 / 2} r, \\
& 2^{-1} d R_{0}^{2}>D\left(2^{-1} R_{0}^{2}+r^{2}\right)^{1 / 2} r+(\alpha / \beta)(1+\theta)(\delta d) R_{0} \mu_{10}
\end{aligned}
$$

If $\rho_{1}, \rho_{2}$ denote the differences between first and second members in the last two inequalities, then $M_{1}=\alpha \rho_{1}, M_{2}=\beta \rho_{2}$, and we shall further require that

$$
\begin{aligned}
& \|\phi\|_{L_{2}}+2^{1 / 2} \pi C \leqslant r, \\
& \alpha R_{0} \mu_{20}\|\phi\|_{L_{2}}+\beta R_{0} 2^{-1 / 2}\left\|\phi_{t}\right\|_{L_{2}} \leqslant \min \left[M_{1}, M_{2}\right] .
\end{aligned}
$$


First we take $r$ so small, say $r \leqslant r_{1}$, that $2 \mu_{0} r<\delta-b$, or $2 \mu_{0} r \leqslant 2 \mu_{0} r_{1}<\delta-b$, and we can take $R_{0} \Gamma=b+\mu_{0} r$. If we assume $R_{0} \geqslant 2^{1 / 2} r$, then $R_{0}^{2} \geqslant 2^{-1} R_{0}^{2}+r^{2} \geqslant 2^{-1} R_{0}^{2}$, and relations (12.10-12.11) become, in a stronger form,

$$
\begin{aligned}
& 0<\eta<2 \pi^{2}, \quad R_{0} \Gamma=\mu_{0} r+b, \quad R_{0} \geqslant 2^{1 / 2} r, \\
& R_{0} \omega_{0}(\sqrt{2 \eta / \pi})<\delta-b-2 \mu_{0} r, \quad 0<r \leqslant r_{1}, \quad 2 \mu_{0} r_{1}<\delta-b, \\
& (1-\varepsilon)(b d)\left(\mu_{0} r+b\right) \eta>(1+\theta)(\delta d) r+(\beta / \alpha) d^{\prime} R_{0}^{2}+(\beta / \alpha) D R_{0} r \\
& 2^{-1} d R_{0}^{2}>D R_{0} r+(\alpha / \beta)(1+\theta)(\delta d) R_{0} \mu_{10}, \\
& \|\phi\|_{L_{2}}+2^{1 / 2} \pi(1+\theta)(\delta d) \leqslant r, \\
& \alpha R_{0} \mu_{20}\|\phi\|_{L_{2}}+\beta R_{0} 2^{-1 / 2}\left\|\phi_{r}\right\|_{L_{2}} \leqslant \min \left[M_{1}, M_{2}\right] .
\end{aligned}
$$

Let us assume now $C \leqslant C_{0}=2^{-3 / 2} \pi^{-1} r$, so that $2^{1 / 2} \pi C \leqslant 2^{-1} r$, and $d \leqslant d_{0}$ with $(1+\theta) \delta d_{0}$ $=2^{-3 / 2} \pi^{-1} r$, hence $d \leqslant 2^{-3 / 2} \pi^{-1}(1+\theta)^{-1} \delta^{-1} r$.

Relations (12.12) take now the stronger form

$$
\begin{aligned}
& 0<\eta<2 \pi^{2}, \quad R_{0} \Gamma=\mu_{0} r+b, \quad R_{0} \geqslant 2^{1 / 2} r, \\
& R_{0} \omega_{0}(\sqrt{2 \eta / \pi}) \leqslant \delta-b-2 \mu_{0} r, \quad 0<r \leqslant r_{1}, \quad 2 \mu_{0} r_{1}<\delta-b, \\
& (1-\varepsilon) b^{2} d \eta+(1-\varepsilon) b \mu_{0} d r>(1+\theta)(\delta d) r+(\beta / \alpha) d^{\prime} R_{0}^{2}+(\beta / \alpha) D R_{0} r \\
& 2^{-1} d R_{0}>D r+(\alpha / \beta)(1+\theta) \delta d \mu_{10}, \\
& \|\phi\|_{L_{2}} \leqslant r / 2, \\
& \|\phi\|_{L_{2}}+2^{-1 / 2}(\beta / \alpha) \mu_{20}^{-1}\|\phi\| \leqslant \alpha^{-1} R_{0}^{-1} \mu_{20}^{-1} \min \left[M_{1}, M_{2}\right] .
\end{aligned}
$$

We take now

$$
d^{\prime}=k^{\prime} d, \quad d^{\prime \prime}=k^{\prime \prime} d, \quad D=k d, \quad k=\max \left[k^{\prime}, k^{\prime \prime}\right],
$$

so that relations (12.13) take the form

$$
\begin{aligned}
& d \leqslant 2^{-3 / 2} \pi^{-1}(1+\theta)^{-1} \delta^{-1} r, \\
& 0<\eta<2 \pi^{2}, \quad R_{0} \Gamma=\mu_{0} r+b, \quad R_{0} \geqslant 2^{1 / 2} r, \\
& R_{0} \omega_{0}(\sqrt{2 \eta / \pi}) \leqslant \delta-b-2 \mu_{0} r, \quad 0<r \leqslant r_{1}, \quad 2 \mu_{\left(r_{1}\right.}<\delta-b, \\
& (1-\varepsilon) b^{2} \eta+(1-\varepsilon) b \mu_{0} r>(1+\theta) \delta r+(\beta / \alpha) k R^{2}+(\beta / \alpha) k R_{0} r \\
& 2^{-1} R_{0}>k r+(\alpha / \beta)(1+\theta) \delta \mu_{10}, \\
& \|\phi\|_{L_{2}} \leqslant r / 2, \\
& \|\phi\|_{L_{2}}+2^{-1 / 2}(\beta / \alpha) \mu_{20}^{-1}\left\|\phi_{n}\right\| \leqslant \alpha^{-1} R_{0}^{-1} \mu_{20}^{-1} \min \left[M_{1}, M_{2}\right] .
\end{aligned}
$$

First, we take $r_{1}>0$ so small that $2 \mu_{0} r_{\mathrm{l}}<\delta-b$, and then we take $0<\eta<2 \pi^{2}$ so small and $R_{0}>0$ so large that

$$
R_{0} \omega_{0}(\sqrt{2 \eta / \pi})=\delta-b-2 \mu_{0} r_{1}, \quad 2^{-3} R_{0} \geqslant(\alpha / \beta)(1+\theta) \delta \mu_{10} .
$$


Now we can take $0<r \leqslant r_{1}$ so small that

$$
\begin{aligned}
& 2^{1 / 2} r \leqslant R_{0}, \quad k r \leqslant 2^{-3} R_{0}, \\
& {\left[(1+\theta) \delta+(\beta / \alpha) k R_{0}-(1-\varepsilon) b \mu_{0}\right] r \leqslant 4^{-1}(1-\varepsilon) b^{2} \eta .}
\end{aligned}
$$

The latter requirement is trivially satisfied if the bracket is $\leqslant 0$. We shall now take $0 \leqslant k^{\prime} \leqslant$ $k_{0}^{\prime}$ with $(\beta / \alpha) k_{0}^{\prime} R_{0}^{2}=4^{-1}(1-\varepsilon) b^{2} \eta$. Having so fixed $R_{0}, r>0$ we take $\Gamma>0$ so that $R_{0} \Gamma=$ $\mu_{0} r+b$. Finally, we may take $d=2^{-3 / 2} \pi^{-1}(1+\theta)^{-1} \delta^{-1} r, k^{\prime}=\min \left[k_{0}^{\prime}, k^{\prime \prime}\right], k^{\prime \prime}=k, d^{\prime}=k^{\prime} d$, $d^{\prime \prime}=D=k d$. Note that $\varepsilon, \theta$ and $k$ are arbitrary, and so are $\alpha$ and $\beta$ positive constants. The first four relations (12.13) are thereby satisfied. Now

$$
\begin{aligned}
\rho_{1} & =d\left\{(1-\varepsilon) b^{2} \eta-\left[(1+\theta) \delta+(\beta / \alpha) k R_{0}-(1-\varepsilon) b \mu_{0}\right] r-(\beta / \alpha) k^{\prime} R_{0}^{2}\right\} \\
& \geqslant d\left[(1-\varepsilon) b^{2} \eta-4^{-1}(1-\varepsilon) b^{2} \eta-4^{-1}(1-\varepsilon) b^{2} \eta\right]=2^{-1}(1-\varepsilon) b^{2} d \eta \\
\rho_{2} & =d R_{0}\left[2^{-1} R_{0}-k r-(\alpha / \beta)(1+\theta) \delta \mu_{10}\right] \\
& \geqslant d R_{0}\left[2^{-1} R_{0}-2^{-3} R_{0}-2^{-3} R_{0}\right]=2^{-2} d R_{0}^{2}, \\
M_{1} & =\alpha \rho_{1} \geqslant 2^{-1} \alpha(1-\varepsilon) b^{2} d \eta, \quad M_{2}=\beta \rho_{2} \geqslant 2^{-2} \beta d R_{0}^{2},
\end{aligned}
$$

and the last two relations (12.13) yield $A_{0}$ and $B_{0}$ :

$$
\begin{aligned}
B_{0} & =\alpha^{-1} R_{0}^{-1} \mu_{20}^{-1} \min \left[M_{1}, M_{2}\right] \\
& =\min \left[\alpha^{-1} R_{0}^{-1} \mu_{20}^{-1} 2^{-1} \alpha(1-\varepsilon) b^{2} d \eta, \alpha^{-1} R_{0}^{-1} \mu_{20}^{-1} 2^{-2} \beta d R_{0}^{2}\right] \\
& =\min \left[2^{-1}(1-\varepsilon) \mu_{20}^{-1} R_{0}^{-1} b^{2} d \eta, 2^{-2}(\beta / \alpha) \mu_{20}^{-1} R_{0} d\right] \\
& =2^{-2} \mu_{20}^{-1} d \min \left[2(1-\varepsilon) R^{-1} b^{2} \eta,(\beta / \alpha) R_{0} d\right], \\
A_{0} & =2^{-1 / 2}(\beta / \alpha) \mu_{20}^{-1} .
\end{aligned}
$$

All relations (12.13) are now satisfied, if

$$
\|\phi\| \leqslant r / 2, \quad\|\phi\|+A_{0}\left\|\phi_{\|}\right\| \leqslant B_{0} .
$$

If we take $B_{0} \leqslant r / 2$ then the first of these relations is included in the second one, and (12.iii) is proved.

Remark. Note that in the proof above, we have treated $\varepsilon, \theta, k^{\prime \prime}=k, \alpha, \beta, b, \delta$ as arbitrary but fixed constants, and then we have determined $r_{1}$ so as $2 \mu_{0} r_{1} \leqslant \delta-b$, then we have determined $R_{0}$ and $\eta$ so that

$$
R_{0} \omega_{0}\left(\sqrt{2 \eta / \pi}=\delta-b-2 \mu_{0} r_{1}, \quad 2^{-3} R_{0} \geqslant(\alpha / \beta)(1+\theta) \delta \mu_{10}\right.
$$

Then we have determined $r$ so that

$$
\begin{aligned}
& 2^{1 / 2} r \leqslant R_{0}, \quad r \leqslant r_{1}, \quad k r \leqslant 2^{-3} R_{0}, \\
& {\left[(1+\theta) \delta+(\beta / \alpha) k R_{0}-(1-\varepsilon) b \mu_{0}\right] r<2^{-2}(1-\varepsilon) b^{2} \eta .}
\end{aligned}
$$

Then we have determined $k_{0}^{\prime}$ so as

$$
(\beta / \alpha) k_{0}^{\prime} \leqslant 2^{-2}(1-\varepsilon) b^{2} \eta, \quad k^{\prime}=\min \left[k_{0}^{\prime}, k^{\prime \prime}\right],
$$


and $\Gamma$ so as $R_{0} \Gamma=\mu_{0} r+b$. Finally, we have

$$
\begin{aligned}
& d \leqslant d_{0}=2^{-3} \pi^{-1}(1+\theta)^{-1} \delta^{-1} r, \quad C=(1+\theta) d \delta=2^{-3 / 2} \pi^{-1} r, \\
& B=(1-\varepsilon) b d, \quad d^{\prime \prime}=k^{\prime} d, \quad d^{\prime \prime}=D=k d,
\end{aligned}
$$

and we have obtained a full set of compatible constants.

\section{ESTIMATION OF $\omega_{0}$ FOR THE ELEMENTS OF $A_{21}$}

(a) First estimates

Any element $u(t, x)$ of $A_{21}$ has Fourier series

$$
u(t, s)=\sum_{l=1}^{\infty} \pi^{-1} \sin l x+\sum_{k=1}^{\infty} \sum_{l=1}^{\infty}\left[c_{k l} 2^{1 / 2} \pi^{-1} \cos k t \sin l x+d_{k} 2^{1 / 2} \pi^{-1} \sin k t \sin l x\right]
$$

and hence

$$
\begin{aligned}
\|u\|_{21}^{2} & =\iint_{G}\left[\left(D_{i} u\right)^{2}+\left(D_{x x} u\right)^{2}\right] \mathrm{d} t \mathrm{~d} x \\
& =\sum_{l} c_{0 l}^{2} l^{4}+\sum_{k} \sum_{l}\left(c_{k l}^{2}+d_{k l}^{2}\right)\left(k^{2}+l^{4}\right) .
\end{aligned}
$$

Then

$$
\begin{aligned}
\left|u(t, x)-u\left(t^{\prime}, x^{\prime}\right)\right| & =\sum_{l} c_{0}\left(\pi^{-1} \sin l x-\pi^{-1} \sin l x^{\prime}\right) \\
& +\sum_{k} \sum_{l}\left[c_{k l}\left(2^{1 / 2} \pi^{-1} \cos k t \sin l x-2^{1 / 2} \pi^{-1} \cos k t^{\prime} \sin l x^{\prime}\right)\right. \\
& +d_{k l}\left(2^{1 / 2} \pi^{-1} \sin k t \sin l x-2^{1 / 2} \pi^{-1} \sin k t^{\prime} \sin l x^{\prime}\right)
\end{aligned}
$$

If we denote by $\Sigma_{N}$ the sum of all terms in (13.1) with $l^{4} \leqslant N$ or $k^{2}+l^{4} \leqslant N$, and by $R_{N}$ the remaining terms, then

$$
\begin{gathered}
u(t, x)=\sum_{N}(t, x)+R_{N}(t, x) \\
\left|u(t, x)-u\left(t^{\prime}, x^{\prime}\right)\right| \leqslant\left|\sum_{N}(t, x)-\sum_{N}\left(t^{\prime}, x^{\prime}\right)\right|+\left|R_{N}(t, x)\right|+\left|R_{N}\left(t^{\prime}, x^{\prime}\right)\right|
\end{gathered}
$$

The term $\left|\Sigma_{N}(t, x)-\Sigma_{N}\left(t^{\prime}, x^{\prime}\right)\right|$ can be written as follows

$$
\begin{aligned}
& \left|\sum_{N}(t, x)-\sum_{N}\left(t^{\prime}, x^{\prime}\right)\right|=\mid \sum_{l^{4} \leqslant N} c_{0 l} l^{2} \cdot l^{-2} \pi^{-1}\left(\sin l x-\sin l x^{\prime}\right) \\
& \quad+\sum_{k^{2}+l^{4} \leqslant N} c_{k l}\left(k^{2}+l^{4}\right)^{1 / 2} \cdot\left(k^{2}+l^{4}\right)^{-1 / 2}\left(2^{1 / 2} \pi^{-1}\right)\left(\cos k t \sin l x-\cos k t^{\prime} \sin l x^{\prime}\right) \\
& \quad+\sum_{k^{2}+l^{4} \leqslant N} d_{k l}\left(k^{2}+l^{4}\right)^{1 / 2} \cdot\left(k^{2}+l^{4}\right)^{-1 / 2}\left(2^{1 / 2} \pi^{-1}\right)\left(\sin k t \sin l x-\sin k t^{\prime} \sin l x^{\prime}\right) \mid,
\end{aligned}
$$

where the trigonometrical expressions are in absolute value $\leqslant l\left|x-x^{\prime}\right|$ and $\leqslant k\left|t-t^{\prime}\right|+$ $l\left|x-x^{\prime}\right|$ respectively. In any case they are $\leqslant l|P-Q|$ and $\leqslant(k+l)|P-Q|$ respectively, $P=(t, x), Q=\left(t^{\prime}, x^{\prime}\right)$. By Schwartz inequality we have 


$$
\begin{aligned}
\left|\sum_{N}(t, x)-\sum_{N}\left(t^{\prime}, x^{\prime}\right)\right| & \left.\leqslant \sum_{l^{4} \leqslant N} c_{0 l}^{2} l^{4}+\sum_{k^{2}+l^{4} \leqslant N}\left(c_{k l}^{2}+d_{k l}^{2}\right)\left(k^{2}+l^{4}\right)\right]^{1 / 2} \\
& \times\left[\sum_{l^{2} \leqslant N} \pi^{-2} l^{-4}+\sum_{k^{2}+l^{4} \leqslant N}\left(k^{2}+l^{4}\right)^{-1}\left(2 \pi^{-2}\right)\left(2 k^{2}+2 l^{2}\right)^{1 / 2}|P-Q|\right. \\
& \leqslant\|u\|_{21}\left[\pi^{-2} \sum_{l^{\prime} \leqslant N} l^{-2}+4 \pi^{-2} \sum_{k^{2}+l^{4} \leqslant N}(1)\right]^{1 / 2}|P-Q|
\end{aligned}
$$

Since $\Sigma l^{-2}=\pi^{2} / 6$ and the number of terms in the sccond sum is $\leqslant$ the number of terms of the sum $\Sigma_{k^{2} \leqslant N} \Sigma_{l^{4} \leqslant N}$, we have

$$
\begin{aligned}
\left|\sum_{N}(t, x)-\sum_{N}(t, x)\right| & \leqslant\|u\|_{21}\left[6^{-1}+4 \pi^{-2} N^{1 / 2} N^{1 / 4}\right]^{1 / 2}|P-Q| \\
& \leqslant\|u\|_{21}\left[6^{-1}+4 \pi^{-2} N^{3 / 4}\right]^{1 / 2}|P-Q|
\end{aligned}
$$

where $6^{-1}=0.16666,4 \pi^{-2}=0.40528$ and $(0.571951)^{1 / 2}=0.75627$. Hence,

$$
\left|\sum_{N}(t, s)-\sum_{N}\left(t^{\prime}, x^{\prime}\right)\right| \leqslant\|u\|_{21}(0.75627) N^{3 / 8}|P-Q|=S_{N}
$$

Concerning $R_{N}$ we have analogously

$$
\begin{aligned}
\left|R_{N}(x)\right| & =\mid \sum_{l^{4}>N} c_{0 l} l^{2} \cdot l^{-2} \pi^{-1} \sin l x \\
& +\sum_{k^{2}++^{4}>N} c_{k l}\left(k^{2}+l^{4}\right)^{1 / 2} \cdot\left(k^{2}+l^{4}\right)^{-1 / 2} 2 \pi^{-1} \cos k t \sin l x \\
& +\sum_{k^{2}+l^{4}>N} d_{k l}\left(k^{2}+l^{4}\right)^{1 / 2} \cdot\left(k^{2}+l^{4}\right)^{-1 / 2} 2 \pi^{-1} \sin k t \sin l x \mid \\
& \leqslant\left(\sum_{l^{\prime}>N} c_{0 l}^{2} l^{4}+\sum_{k^{2}+l^{4}>N}\left(c_{k l}^{2}+d_{k l}^{2}\right)\left(k^{2}+l^{4}\right)\right)^{1 / 2} \\
& \times\left(\sum_{l^{4}>N} \pi^{-2} l^{-4}+\sum_{k^{2}+l^{4}>N} 2 \pi^{-2} \cdot 2\left(k^{2}+l^{4}\right)^{-1}\right)^{1 / 2} \\
& \leqslant\|u\|_{21}\left(\pi^{-2} \sum_{l^{4}>N} l^{-4}+4 \pi^{-2} \sum_{k^{2}+l^{4}>N}\left(k^{2}+l^{4}\right)^{-1}\right)^{1 / 2}=R_{N} .
\end{aligned}
$$

We now have proved that, for $P=(t, x), Q=\left(t^{\prime}, x^{\prime}\right)$, we have for any $N$,

$$
|u(P)-u(Q)| \leqslant S_{N}+2 R_{N}
$$

(b) Evaluation of $\int_{0}^{\pi / 2}(\sin \theta)^{-1 / 2} \mathrm{~d} \theta$

From [3, p. 171, No. 287.50], we have

$$
\int_{0}^{\phi}(\sin 2 a \theta)^{-1 / 2} \mathrm{~d} \theta=g F(A, k)
$$

for $k=2^{-1 / 2}, g=\sqrt{2 / a}, A=\arcsin \left((2 \sin a \phi)^{1 / 2}(1+\cos a \phi+\sin a \phi)^{-1 / 2}\right)$, where $F$ is the incomplete elliptic integral of the first kind. For $a=1 / 2, \phi=\pi / 2$, then $g=2 \sqrt{2}=2 \cdot 82842$, 
$a=\pi / 4$, and

$$
A=\arcsin \left(2^{1 / 2}(2+\sqrt{2})^{-1 / 2}\right)=\arcsin (0.76537)=49^{\circ}, 56.372 .
$$

From [3, Table on p. 328], $F=0.927053$, and

$$
\int_{0}^{\pi / 2}(\sin \theta)^{-1 / 2} \mathrm{~d} \theta=g F=2.62210 .
$$

(c) Evaluation of $R_{N}$

For $N \geqslant 1, k \geqslant 2, l \geqslant 2, k^{2}+l^{4}>N$ we have

$$
(k-1)^{2}+(l-1)^{4}=K^{2}\left(1-k^{-1}\right)^{2}+l^{4}\left(1-l^{-1}\right)^{4} \geqslant 2^{-2} k^{2}+(5 / 16) l^{4}>2^{-2} N .
$$

Hence, by reduction to a double integral, and usual transformations, $y^{2}=z$, and $x=\rho \cos \theta$, $z=\rho \sin \theta$, we also have

$$
\begin{aligned}
& \sum_{k^{2}+l^{4}>N, k \geqslant 2, l \geqslant 2}\left(k^{2}+l^{4}\right)^{-1}<\int_{x^{2}+y^{4} \geqslant N / 4, x \geqslant 0, y \geqslant 2}\left(x^{2}+y^{4}\right)^{-1} \mathrm{~d} x \mathrm{~d} y \\
& =\int_{x^{2}+z^{2} \geqslant N / 4, x \geqslant 0, z \geqslant 0}\left(x^{2}+z^{2}\right)^{-1}\left(2 z^{-1 / 2}\right) \mathrm{d} x \mathrm{~d} z \\
& =2^{-1} \int_{0}^{\pi / 2}(\sin \theta)^{-1 / 2} \int_{(N / 4)^{1 / 2}}^{+\infty} \rho^{-3 / 2} \mathrm{~d} \rho=2^{1 / 2}(2.62210) N^{-1 / 4}=3.70820 N^{-1 / 4}
\end{aligned}
$$

On the other hand, we have

$$
\begin{aligned}
\sum_{k^{2}+l^{4}>N, k=1 \text { or } l=1}\left(k^{2}+l^{4}\right)^{-1} & =\sum_{l>1, l^{4}>N-1}\left(1+l^{4}\right)^{-1}+\sum_{k>1, k^{2}>N-1}\left(1+k^{2}\right)^{-1} \\
< & \Sigma l^{-4}+\Sigma k^{-2} \leqslant \int_{(N-1)^{1 / 4}}^{+\infty} \rho^{-4} \mathrm{~d} \rho+\int_{(N-1)^{1 / 2}-1}^{+\infty} \rho^{-2} \mathrm{~d} \rho \\
& \left.=3^{-1}\left(N^{-1 / 4}\left((N-1)^{1 / 4}-1\right)^{3}\right)^{-1} N^{-1 / 4}+N^{1 / 4}\left((N-1)^{1 / 2}-1\right)^{-1}\right) N^{-1 / 4}
\end{aligned}
$$

For $N=10$ the factors of $N^{-1 / 4}$ are 1.51104 and 0.88919 respectively. From analysis we know that $\Sigma_{1}^{\infty} l^{-4}=1.08232, \Sigma_{1}^{\infty} k^{-2}=1.64493$. It is easy to verify that the sums of the two series above are $<(1.51104) N^{-1 / 4}$ and $<(0.88919) N^{-1 / 4}$ for $N=1,2, \ldots, 9$. Since $3.70820+1.51104$ $+0.88919=6.10843$, we have $\Sigma_{k^{2}+l^{4}>N}\left(k^{2}+l^{4}\right)^{-1} \leqslant(6.10843) N^{-1 / 4}$ for all $N$.

The same computations have also shown that

so that

$$
\sum_{r>N} l^{-4}<(1.51104) N^{-1 / 4}
$$

$$
\begin{aligned}
R_{N} & =\|u\|_{21}\left[\pi^{-2} \sum_{N^{\prime}>N} l^{-4}+4 \pi^{-2} \sum_{k^{2}+\mu^{4}>N}\left(k^{2}+l^{4}\right)^{-1}\right]^{1 / 2} \\
& \leqslant\|u\|_{21} \pi^{-1}(1.51104+4 \cdot(6.10843))^{1 / 2} N^{-1 / 8} \\
& =\|u\|_{21}(1.62134) N^{-1 / 8}
\end{aligned}
$$

(d) Estimate of the modulus of continuity

First let us assume that we have in general

$$
|u(P)-u(Q)| \leqslant S_{N}+2 R_{N}, \quad S_{N}=B N^{\beta}|P-Q|, \quad R_{N}=A N^{-\alpha}
$$


and given constants $A, B, \alpha, \beta>0$. By taking

$$
A N^{-\alpha}=(\varepsilon / 3)|P-Q|^{\gamma}, \quad B N^{\beta}|P-Q|=(\varepsilon / 3)|P-Q|^{\gamma}
$$

for suitable constants $\varepsilon>0, \gamma>0$, we derive

$$
B N^{\beta}|P-Q|=A N^{-\alpha}
$$

hence $N^{\alpha+\beta}=(A / B)|P-Q|^{-1}$, and by computation

$$
A N^{-\alpha}=B N^{\beta}|P-Q|=A^{\beta(\alpha+\beta)^{-1}} B^{\alpha(\alpha+\beta)^{-1}}|P-Q|^{\alpha(\alpha+\beta)^{-1}} \text {. }
$$

Thus, $\gamma=\alpha(\alpha+\beta)^{-1}, \varepsilon=3 A^{\beta(\alpha+\beta)^{-1}} B^{\alpha(\alpha+\beta)^{-1}}$, and

$$
|u(P)-u(Q)| \leqslant 3 A^{\beta(\alpha+\beta)^{-1}} B^{\alpha(\alpha+\beta)^{-1}}|P-Q|^{\alpha(\alpha+\beta)^{-1}} .
$$

In this case above we have $A=\|u\|_{21}(1.62134), \alpha=1 / 8, B=\|u\|_{21}(0.75627), \beta=3 / 8$. Hence $\alpha+\beta=1 / 2, \alpha(\alpha+\beta)^{-1}=1 / 4, \beta(\alpha+\beta)^{-1}=3 / 4$,

$$
\begin{aligned}
|u(P)-u(Q)| & \leqslant\|u\|_{21}\left[3(1.62134)^{3 / 4}(0.75627)^{1 / 4}\right]|P-Q|^{1 / 4} \\
& =\|u\|_{21}(4.0196)|P-Q|^{1 / 4}
\end{aligned}
$$

(e) Estimates for $\mu_{0}, \mu_{10}, \mu_{20}$

Here $\|u\|_{\infty}$ is given by the same expression for $R_{N}$ where the sums range over all possible values of $l$ and $k$, that is,

$$
|u(t, x)| \leqslant\left\|u_{21}\right\|\left(\pi^{-2} \sum_{1}^{\infty} l^{4}+4 \pi^{-2} \sum_{1}^{\infty} \sum_{1}^{\infty}\left(k^{2}+l^{4}\right)^{-1}\right)^{1 / 2}
$$

where the first sum is 1.08237 and the second one can be written as

$$
\left(\sum_{k^{2}+\mu \geqslant 1, k \geqslant 2 . l \geqslant 2}+\sum_{k^{2}+l^{4} \geqslant 1 . k=1 \text { or } l=1}\right)\left(k^{2}+l^{4}\right)^{-1}
$$

and these two sums are given in (c) for $N=1$. Then

$$
\|u\|_{\infty} \leqslant\|u\|_{21} \pi(1.08237+4 \cdot 6.10843)^{1 / 2}=\left\|u_{21}\right\|(1.62134)
$$

In other words, we can take $\mu_{0}=1.62134$, and then we can take $\mu_{10}=\mu_{0} a=2 \pi^{2} \mu_{0}=32.004$.

\section{ANALYSIS IN THE LARGE OF THE DOUBLY PERIODIC SOLUTIONS OF THE} WAVE EQUATION $u_{u}-u_{x x}=f(t, x, u)$

We consider here the problem of the solutions $u(t, x)$, periodic in $t$ and $x$, of the hyperbolic problem

$$
\begin{aligned}
u_{t}-u_{x x} & =f(t, x, u), \quad(t, x) \in \mathbb{R}^{2}, \\
u(t+2 \pi, x) & =u(t, x)=u(t, x+2 \pi) .
\end{aligned}
$$

Let $G=[0,2 \pi] \times[0,2 \pi]$, let $a=$ meas $G=4 \pi^{2}$, and let $\|u\|_{L_{2}},(u, v)$ denote the usual square norm and inner product in $L_{2}(G)$.

Let $\left[e_{k l}, k, l=0,1, \pm 1, \pm 2, \ldots\right]$ denote the system generated by $\exp (i k t) \exp (i l x)$ in $\mathbb{R}^{2}$ and orthogonal in $G$. Then any element $u \in X=A_{11}$ has Fourier series

$$
u(t, x)=\sum_{k l} b_{k l} e_{k l}, \quad b_{k l}=\left(u, e_{k l}\right)
$$


with

$$
\begin{aligned}
& \|u\|_{X}=b_{00}^{2}+\left\|u_{d}\right\|_{L_{2}}^{2}+\left\|u_{x}\right\|_{L_{2}}^{2}=b_{00}^{2}+\sum_{k, l} b_{k l}^{2}\left(k^{2}+l^{2}\right)<+\infty \\
& \|u\|_{X}=(u, u)_{X}^{1 / 2}, \quad(u, v)_{X}=b_{00} \mathcal{C}_{00}+\left(u_{t}, v_{t}\right)_{L_{2}}+\left(u_{x}, v_{x}\right)_{L_{2}}
\end{aligned}
$$

By the same arguments as in Section 6 we know that $u \in L_{q}$ for any $q, 1 \leqslant q<+\infty$, $u_{t}, u_{x} \in L_{2}$, and $\|u\|_{L_{q}} \leqslant \mu_{1 q}\|u\|_{X},\left\|u_{t}\right\|_{L_{2}} \leqslant\|u\|_{X},\left\|u_{x}\right\|_{L_{2}} \leqslant\|u\|_{X}$. Let $X_{0}$ denote the set of all elements in $X$ with Fourier series $\Sigma_{k^{2}=\imath} b_{k l} e_{k l}$. We know from Section 7 that these elements are bounded continuous and Lipschitzian in $\mathbb{R}^{2}$ with

$$
\begin{aligned}
& \|u\| \leqslant \mu_{0}\|u\|_{X}, \quad|u(t+h, x)-u(t, x)| \leqslant \mu_{1}|| u \|_{X}|h|, \\
& |u(t, x+k)-u(t, x)| \leqslant \mu_{1}\|u\|_{X}|k|,
\end{aligned}
$$

for some constants $\mu_{0}, \mu_{1}$ which we estimated in Section 7.

Let $E$ denote the operator defined by $E u=u_{t t}-u_{x x}$. Let $D$ denote the class of all elements $u$ of class $C^{\infty}$ in $\mathbb{R}^{2}$ and $2 \pi$-periodic in $t$ and $x$. By a weak solution $u \in X=A_{11}$ of (14.1) we denote any element $u \in X$ such that

$$
\left(u, y_{t r}-y_{x x}\right)_{L_{2}}=(f(t, x, u), y) \text { for all } y \in D .
$$

Thus, the subspace $X_{0}$ of $X$ can be interpreted as the set of all elements $u \in X$ for which $\left(u, y_{t t}-y_{x x}\right)_{L_{2}}=0$ for all $y \in D$, that is, the weak kernel of $E$. Then, for every element $u^{*}$ $\in X_{0}$ we also have, integrating by parts, $\left(u_{t}^{*}, y_{t}\right)=\left(u_{x}^{*}, y_{x}\right)$. If $v^{*} \in X_{0}$ and we approximate $v^{*}$ by elements $y \in D$ in $X$, thus $y_{t}, y_{x}$ approximate $v_{t}^{*}, v_{x}^{*}$ in $L_{2}$, then we also have

$$
\left(u_{t}^{*}, v_{t}^{*}\right)_{L_{2}}=\left(u_{x}^{*}, v_{x}^{*}\right)_{L_{2}}, \quad\left\|u_{t}^{*}\right\|_{L_{2}}=\left\|u_{x}^{*}\right\|_{L_{2}}=2^{-1}\left[\left\|u^{*}\right\|_{X}^{2}-\left(u_{00}\right)^{2}\right] \text {. }
$$

For any element $u^{*} \in X_{0}$ we have, therefore

$$
\begin{aligned}
& \left(u^{*}, v^{*}\right)_{X}=u_{00}^{*} v_{00}^{*}+\left(u_{t}^{*}, v_{t}^{*}\right)_{L_{2}}+\left(u_{x}^{*}, v_{x}^{*}\right)_{L_{2}}=u_{00}^{*} v_{00}^{*}+2\left(u_{t}^{*}, v_{t}^{*}\right)_{L_{2}}, \\
& \left\|u^{*}\right\|_{X}=\left(u_{00}^{*}\right)^{2}+2\left\|u_{t}^{*}\right\|^{2} .
\end{aligned}
$$

Let $P$ denote the natural projection of $X$ onto $X_{0}$. For $u \in X_{1}=(I-P) X$, then $u=$ $\Sigma b_{k l} e_{k l}$ with $\Sigma b_{k l}^{2}\left(k^{2}+l^{2}\right)<+\infty$, where $\Sigma$ ranges over all $k, l=0, \pm 1, \pm 2, \ldots$ with $k^{2} \neq l^{2}$. Let $Y_{0}, Y_{1}$ denote the analogous decomposition of $Y=L_{2}(G)$, and let $Q$ denote the natural projection of $Y$ onto $Y_{0}$. We can now define the operator $H: Y_{1} \rightarrow X_{1}$. Indeed, for $u \in$ $Y_{1}$, or $u=\Sigma_{k^{2} \neq l^{2}} b_{k l} e_{k l}$ with $\Sigma b_{k l}^{2}<+\infty$, let $v=H u=\Sigma_{k^{2} \neq l^{2}} b_{k l}\left(-k^{2}+l^{2}\right)^{-1} e_{k l}$. Then, $v=H u \in$ $(I-P) X$ is the weak solution of $E v=u$. Moreover, $\|H u\|_{L_{2}} \leqslant\|u\|_{L_{2}}$ and $\|H u\|_{X} \leqslant\|u\|_{L_{2}}$ for $u \in Y_{1}$ as we proved in Section 7. Thus for the linear operator $H: Y_{1} \rightarrow X_{1}$ we have $\|H\|=$ $L=1$. With $X, Y, P, Q, H$ as above, axioms (a), (b), (c) of Section 3 are satisfied. We now define the finite dimensional subspaces $X_{0 n}$ of $X_{0}, X_{1 n}$ of $X_{1}, Y_{0 n}$ of $Y_{0}$ as in Section 12, and the natural projections $R_{n}: X_{1} \rightarrow X_{1 n}, S_{n}: X_{0} \rightarrow X_{0 n}, S_{n}^{\prime}: Y_{0} \rightarrow Y_{0 n}$ as in Section 12. Finally, let $\alpha_{n}: Y_{0 n} \rightarrow X_{0 n}$ denote the linear map defined by $\alpha_{n} y=\Sigma_{0 n} b_{k l} e_{k l}$, where $y=\Sigma_{0 n} b_{k l} e_{k l}, y \in$ $Y_{0 n}$, where $\Sigma_{0 n}$ ranges over all $k, l=0, \pm 1, \ldots, \pm n$ with $k^{2}=R^{2}$. Hence $\alpha_{n}^{-1}(0)=0$, and equation $\alpha_{n} S_{n}^{\prime} Q N u=0$ is equivalent to $S_{n}^{\prime} Q N u=0$. Moreover, $S_{n}^{\prime} Q N u=0$ if and only if $\left(S_{n}^{\prime} Q N u, v^{*}\right)=0$ for all $v^{*} \in X_{0 n}$. We can repeat on $\alpha_{n}$ the same remarks we made in Section 12.

Let $X_{0 n}^{\prime}$ denote the subspace of all $u \in X_{0 n}$ with mean value zero. For every element $u \in X_{0 n}$ of mean value zero, or $u \in X_{0 n}^{\prime}$, or $u=\Sigma_{0 n}^{\prime} b_{k l} e_{k l}$ where $\Sigma_{0 n}^{\prime}$ ranges over all $k, l=$ 
$\pm 1, \ldots, \pm n$, with $k^{2}=\hat{F}$, let us define $J$ by taking

$$
J u=\Sigma_{0 n}^{\prime} k^{-1} b_{k} e_{k l}^{*},
$$

where $e_{k l}^{*}$ is obtained by $e_{k l}$ by replacing $\cos k t$ by $\sin k t$, and $\sin k t$ by $-\cos k t$. Then, $(J u)_{t}$ $=u$. Let $\alpha, \beta, \gamma$ be nonnegative constants with $\gamma>0, \alpha+\beta>0$. For every element $u \in$ $X_{0 n}$, that is, $u=\Sigma_{0 n} b_{k l} e_{k l}$ where $\Sigma_{0 n}$ ranges over all $k, l=0, \pm 1, \ldots \pm n$ with $k^{2}=l^{2}$ we take

$$
J_{n} u=\gamma b_{00} e_{00}+\sum_{0 n}^{\prime}\left(\alpha k^{-2}+\beta\right) b_{k l} e_{k l} \text {. }
$$

Thus, on $X_{0 n}^{\prime}$ we have $J_{n} u=-\alpha^{2} J^{2}+\beta J$.

The original problem (14.1) is now written in the abstract form $E x=N x$, or in the equivalent form of auxiliary and bifurcation equations

$$
u_{1}=H(I-Q) N u, \quad Q N u=0, \quad u=u^{*}+u_{1} \in X
$$

For every integer $n$, we have then the reduced equations

$$
u_{1}=R_{n} H(I-Q) N u, \quad J_{n} \alpha_{n} S_{n}^{\prime} Q N u=0, \quad u=u^{*}+u_{1} \in X_{n}=X_{0 n}+X_{1 n},
$$

and, for each $n$, we apply Theorem (5.i) and Remark 1 of Section 5 .

Below, we shall show that there are numbers $R_{0}, r>0$ such that, for every integer $n$, there is at least one solution $u_{n} \in X_{n}, u_{n}=u_{n}^{*}+u_{n 1}, u_{n}^{*} \in X_{0 n}, u_{n 1} \in X_{1 n}$, hence $u_{n} \in X$, $u_{n}^{*} \in X_{0}, u_{n 1} \in X_{1}, n=1,2, \ldots$, with $\left\|u_{n}\right\|_{X} \leqslant\left(R_{0}^{2}+r^{2}\right)^{1 / 2}$ for all $n$.

Proceeding as in Section 5, we now introduce the space $\mathscr{X}$. For $\mathscr{X}$ we choose $L_{q}(G)$ for any fixed $q \geqslant 2$. Here $\left\|u_{n}\right\|_{X}$ is bounded in $X=A_{11}$, and the sequence $u_{n}(t, x),(t, x) \in \mathbb{R}^{2}$. is made up of functions in $A_{11}$ with bounded norm in $X=A_{11}$, a real Hilbert space. Hence, there is a subsequence, say still $[n]$, which converges weakly in $X=A_{11}$ to some element $u \in X=$ $A_{11}$. Hence, by Section $5, u_{n}$ converges strongly in $L_{q}$ to $u$, as we have seen in Section 5 . We could take the sequence $[n]$ in such a way that $u_{n} \rightarrow u$ pointwise a.e. in $G$. Now assume for instance that $f(t, x, u)$ is continuous and bounded in $\mathbb{R}^{3}$, and doubly $2 \pi$-periodic in $(t, x)$. Then, $f\left(t, x, u_{n}(t, x)\right) \rightarrow f(t, x, u(t, x))$ pointwise a.e. in $G$, the functions $f\left(t, x, u_{n}(t, x)\right)$ are measurable equibounded functions in $G$, and then $f\left(t, x, u_{n}(t, x)\right) \rightarrow f(t, x, u(t, x))$ in $L_{q}(G)$, or $N u_{n} \rightarrow N u$ in $L_{q}(G)$.

Proceeding to the limit in the coupled equations, we obtain that $u \in X=L_{q}(G)$ is a solution in the weak sense of the original problem (14.1). For the solution $u \in L_{q}(G), u_{t}, u_{x}$ exist in $L_{2}, u_{t t}, u_{x x}$ exist in the distributional sense and they satisfy the original equation in the weak sense.

(14.i) Let $f(t, x, s)$ be of class $C^{1}$ in $\mathbb{R}^{3}, \pi$-periodic in $t$ and $x$, and bounded in $\mathbb{R}^{3}$, say $|f(t, x, s)| \leqslant \gamma_{0}$. Let us assume that there are constants $R_{0}, r$ such that $L \gamma_{0} \leqslant r$ and such that for all $u^{*} \in X_{0}, u_{1} \in X_{1},\|u\|_{X}=R_{0},\left\|u_{1}\right\|_{X} \leqslant r, X=A_{11}$,

$$
\begin{aligned}
\gamma(f(t, x, u))_{00} u_{00}^{*} & +\alpha \int_{G} f(t, x, u) u^{*} \mathrm{~d} t \mathrm{~d} x \\
& +\beta \int_{G}(f(t, x, u))_{t} u_{t}^{*} \mathrm{~d} t \mathrm{~d} x \geqslant 0 \quad \text { or } \leqslant 0
\end{aligned}
$$


Then the hyperbolic problem (14.1) has at least one solution $u(t, x) \in X=A_{11}$ with $\|u\|_{X} \leqslant$ $R=\left(R_{0}^{2}+r^{2}\right)^{1 / 2}$.

Proof. We shall only show that (5.i) applies. Thus, we have to verify hypotheses (a) and (b) of (5.i). Actually, (a) is satisfied, and, by Remark 1 of Section it is enough to verify that

$$
\left(J_{n} \alpha_{n} S_{n}^{\prime} Q N u, u^{*}\right)_{X} \geqslant 0 \quad[\text { or } \leqslant 0],
$$

for all $u^{*} \in X_{0 n},\left\|u^{*}\right\|_{X}=R_{0}, u=u^{*}+u_{1}, u_{1} \in X_{1 n},\left\|u_{1}\right\|_{X} \leqslant r$. Note that, because of the choice of $J_{n}$, and by integration by parts, we have,

$$
\begin{aligned}
\left(J_{n} \alpha_{n} S_{n}^{\prime} Q N u, u^{*}\right) & =\left(J_{n} \alpha_{n} S_{n}^{\prime} Q N u\right)_{00} u_{00}^{*}+2\left(\left(J_{n} \alpha_{n} S_{n}^{\prime} Q N u\right)_{t}, u_{t}^{*}\right)_{L_{2}} \\
& =\gamma(Q N u)_{00} u_{00}^{*}-2 \alpha\left(\left(J^{2} \alpha_{n} S_{n}^{\prime} Q N u\right)_{t}, u_{t}^{*}\right)_{L_{2}}+2 \beta\left(\left(\alpha_{n} S_{n}^{\prime} Q N u\right)_{r^{\prime}} u_{r}^{*}\right)_{L_{2}} \\
& =\gamma(N u)_{00} u_{00}^{*}-2 \alpha\left(J \alpha_{n} S_{n}^{\prime} Q N u, u_{t}^{*}\right)_{L_{2}}+2 \beta\left(\left(\alpha_{n} S_{n}^{\prime} Q N u\right)_{t}, u_{t}^{*}\right)_{L_{2}} \\
& =\gamma(N u)_{00} u_{00}^{*}+2 \alpha\left(\alpha_{n} S_{n}^{\prime} Q N u, u^{*}\right)_{L_{2}}+2 \beta\left(\left(\alpha_{n} S_{n}^{\prime} Q N u\right)_{t} u_{t}^{*}\right)_{L_{2}}
\end{aligned}
$$

Now we have, as in Section 12,

$$
\begin{aligned}
\left(J_{n} \alpha_{n} S_{n}^{\prime} Q N u, u^{*}\right) & =\gamma\left(f(t, x, u)_{00} u_{00}^{*}+2 \alpha \int_{G} f(t, x, u) u^{*} \mathrm{~d} t \mathrm{~d} x\right. \\
& +2 \beta \int_{G}(f(t, x, u))_{t} u_{t}^{*} \mathrm{~d} t \mathrm{~d} x,
\end{aligned}
$$

where $(f(t, x, u))_{t}=f_{t}+f_{u} u_{t}$.

A set of inequalities implying relation (14.4)

Note that for $X=A_{11}$, the elements $u^{*} \in X_{0}$ with $\left\|u^{*}\right\|_{X}=1$ are Lipschitzian functions $u(t, x)$ in $\mathbb{R}^{2}$ with a Lipschitz constant, say $\mu_{1}>0$ which is an absolute constant.

Let $R_{0}, r, \Gamma, \eta, \Lambda$ be positive numbers which we shall determine later.

If $v^{*} \in X_{0},\left\|v^{*}\right\|_{X}=1, \sigma \in X_{1},\|\sigma\|_{X} \leqslant r$, are given elements, then for $0 \leqslant \rho \leqslant R_{0}, \rho v^{*}$ is bounded and Lipschitzian with $\left\|\rho v^{*}\right\|_{\infty} \leqslant \mu_{0} R_{0}$ and Lipschitz constant $\leqslant \mu_{1} R_{0}$, while $\sigma \in L_{q}$ with \|\|$_{L_{q}} \leqslant \mu_{q 1} R_{0}, 1 \leqslant q<+\infty$. Let

$$
\begin{aligned}
G^{\prime} & =\left[(t, x) \in G|| v^{*}(t, x)|\leqslant \Gamma,| \sigma(t, x) \mid \leqslant \Lambda\right] \\
G^{\prime \prime} & =\left[(t, x) \in G|| v^{*}(t, x)|\geqslant \Gamma,| \sigma(t, x) \mid \leqslant \Lambda\right] \\
G^{\prime \prime \prime} & =[(t, x) \in G|| \sigma(t, x) \mid \geqslant \Lambda] .
\end{aligned}
$$

Then, $\|\sigma\|_{L_{q}} \leqslant \mu_{q 1}\|\sigma\|_{X} \leqslant \mu_{q 1} r$, and

$$
\Lambda^{q} \text { meas } G^{\prime \prime \prime} \leqslant \int_{G}|\sigma(t, x)|^{\mid q} \mathrm{~d} t \mathrm{~d} x=\|\sigma\|_{\mathcal{L}_{q}} \leqslant \mu_{q}^{q} r^{q},
$$

or

$$
\text { meas } G^{\prime \prime \prime} \leqslant \Lambda^{-q} \mu_{q 1}^{q} r^{q} \text {. }
$$

Let $k(s), 0 \leqslant s \leqslant D_{0}=\operatorname{diam} \bar{G}$, denote the function defined in $(9 . i)$, so that $k(s)>0$ for $0<s \leqslant D_{0}, k(0)=0$, and for every point $P \in \bar{G}$ and $U(P, s)=\left[Q \in \mathbb{R}^{2}|| Q-P \mid \leqslant s\right]$, we also have meas $[U(P, s) \cap G] \geqslant k(s), 0<s \leqslant D_{0}$. We have seen that it is not restrictive to 
assume $k=k(s)$ continuous in $[0, D]$. Actually, because of the double periodicity, in the present situation, we can take $k=k(s)=\pi s^{2}$, with inverse function $s=s(k)=\left(\pi^{-1} k\right)^{1 / 2}$, and meas $[U(P, s) \cap G] \geqslant \pi s^{2}$ for all $s \geqslant 0$.

(14.ii) Let $f(t, x, u)=\phi(t, x)+g(u)$, where $\phi$ is of class $C^{1}$ and $2 \pi$-periodic in $t$ and $x$, and $g: \mathbb{R} \rightarrow \mathbb{R}$ is of class $C^{1}$. Let us assume that

$$
\begin{aligned}
& u g(u) \geqslant 0, \quad|g(u)| \leqslant C, \quad\left|g_{u}(u)\right| \leqslant D,-d^{\prime} \leqslant g_{u}(u) \leqslant d^{\prime \prime}, \quad u \in \mathbb{R}, \\
& g(u) \geqslant B \quad \text { for } u \geqslant b, \quad g(u) \leqslant-B \quad \text { for } u \leqslant-b, \\
& g(u) \geqslant d>0 \quad \text { for }|u| \leqslant \delta,
\end{aligned}
$$

for suitable positive constants $B, C, d, d^{\prime}, d^{\prime \prime}, b, \delta$ with $b<\delta, B<C, D=\max \left[d^{\prime}, d^{\prime \prime}\right]$.

Let $\Gamma, \eta, r, R_{0}, q, \alpha, \beta, \gamma, \Lambda, \lambda, \tau$ be positive constants such that

$$
\begin{gathered}
M_{1}=\beta d R_{0}^{2} 2^{-1}\left(1-\lambda^{2}\right)-\left(d+d^{\prime}\right) R_{0}^{2} \mu_{1}^{2} \Lambda^{-q} \mu_{q}^{q} r^{q}-D R_{0} 2^{-1 / 2}\left(1-\lambda^{2}\right)^{1 / 2} r \\
-\alpha C R_{0} \mu_{10}-\gamma C R_{0} \lambda>0, \\
M_{2}=\alpha\left(\eta-\Lambda^{-q} \mu_{q}^{q} r^{q}\right) B R_{0} \Gamma-\alpha C \mu_{0} r-\beta d^{\prime} R_{0}^{2} 2^{-1}\left(1-\lambda^{2}\right) \\
-\beta D R_{0} 2^{-1 / 2}\left(1-\lambda^{2}\right)^{1 / 2} r-\gamma\left(4 \pi^{2}\right)^{-1} \mu_{10}^{2} D R_{0} r>0, \\
M_{3}=\gamma B R_{0} \varepsilon-\gamma\left(4 \pi^{2}\right)^{-1} \mu_{10}^{2} D R_{0} r-\alpha C \mu_{10} r-\beta d^{\prime} R_{0}^{2} 2^{-1}\left(1-\lambda^{2}\right) \\
-\beta C R_{0} 2^{-1 / 2}\left(1-\lambda^{2}\right)^{1 / 2} r>0, \\
\quad 2 \pi C<r, \quad \eta>\Lambda^{-q_{1}} \mu_{q 1}^{q} r^{q}, \quad R_{0} \Gamma+R_{0} \mu_{1} \sqrt{\eta / \pi}+\Lambda \leqslant \delta, \\
\quad R_{0} \Gamma-\Lambda \geqslant b, \quad \Gamma \geqslant \mu_{1} \sqrt{2 \pi}, \quad 0<\eta \leqslant a=4 \pi^{2}, \\
\quad 0 \leqslant \lambda \leqslant 1, \quad \lambda \geqslant \pi \mu_{0}\left(1-\lambda^{2}\right)^{1 / 2}+\tau, \quad R_{0} \geqslant b / \tau .
\end{gathered}
$$

Then, for $\rho=R_{0}, v^{*} \in X_{0},\left\|v^{*}\right\|_{X}=1, \sigma \in X_{1},\|d\|_{X} \leqslant r$, we have

If, in addition

$$
\begin{aligned}
\Omega & =\gamma\left[\phi_{00}+\left(g\left(\rho v^{*}+\sigma\right)\right)_{00}\right] v_{00}^{*}+\alpha \int_{G}\left[\phi+g\left(\rho v^{*}+\sigma\right)\right]\left(\rho v^{*}\right) \mathrm{d} t \mathrm{~d} x \\
& +\beta \int_{G}\left[\phi_{t}+\left(g\left(\rho v^{*}+\sigma\right)\right)_{r}\left(\rho v^{*}\right)_{t} \mathrm{~d} t \mathrm{~d} x \geqslant 0 .\right.
\end{aligned}
$$

then problem (14.1) with $f=\phi+g(u)$ has at least a solution

$$
\begin{aligned}
& u \in X=A_{11}, \quad u=u^{*}+u_{1}, \quad u^{*} \in X_{0}, \quad u_{1} \in X_{1}, \\
& \left\|u^{*}\right\|_{X} \leqslant R_{0}, \quad\left\|u_{1}\right\|_{X} \leqslant r, \quad\|u\|_{X} \leqslant R=\left(R_{0}^{2}+r^{2}\right)^{1 / 2} .
\end{aligned}
$$

Proof. Note that, for $u \in X=A_{11},\|u\|_{X} \leqslant R, F(t, x)=f(t, x, u(t, x))=\phi(t, x)+$ $g(u(t, x))$ we certainly have $\|F\|_{Y}=\|F\|_{L_{2}} \leqslant\|\phi\|_{L_{2}}+C(\text { meas } G)^{1 / 2}=\|\phi\|_{L_{2}}+2 \pi C$, and we take $\gamma(s)=\|\phi\|_{2}+2 \pi C$, a constant function. Since $L=\|H\|=1$, requirement $L \gamma(R) \leqslant r$ of $(14 . i)$ reduces to inequality $(14.8)$.

Let us assume $\left|v_{00}^{*}\right| \leqslant \lambda$. 
Note that, for $v^{*} \in X_{0},\left\|v^{*}\right\|_{X}=1$, we have

$$
\left\|v_{t}^{*}\right\|_{L_{2}}^{2}=\left\|v_{x}^{*}\right\|_{L_{2}}^{2}=2^{-1}\left(\left\|v^{*}\right\|_{X}^{2}-v_{00}^{2}\right) \text {, or }\left\|v_{t}^{*}\right\|_{L_{2}}=\left\|v_{x}^{*}\right\|_{L_{2}} \geqslant 2^{-1 / 2}\left(1-\lambda^{2}\right)^{1 / 2} \text {. }
$$

For $\rho=R_{0}, v^{*} \in X_{0}, \sigma \in X_{1},\left\|v^{*}\right\|_{X}=1,\|\sigma\|_{X} \leqslant r$, not only $v^{*}$ and $\sigma$ are orthogonal in $L_{2}$, but also $v_{t}^{*}$ and $\sigma_{t}$. Hence

$$
R_{0}^{2} 2^{-1} \leqslant\left\|\rho v_{l}^{*}+\sigma_{\|}\right\|_{L_{2}}^{2}=\left\|\rho v_{t}^{*}\right\|^{2}+\left\|\sigma_{t}\right\|_{L_{2}}^{2} \leqslant R_{0}^{2} 2^{-1}\left(1-\lambda^{2}\right)+r^{2}
$$

First, let us assume meas $G^{\prime \prime}<\eta$. For $P \in G$ we have meas $(U(P, s) \cap G)>k(s)$ and thus, for $s=s(\eta)$ we also have meas $(U(P, s) \cap G)>k(s)=\eta>$ meas $G^{\prime \prime \prime}$, that is, the ball $U(P$, $s)$ is not filled by points of $G^{\prime \prime} \cup G^{\prime \prime \prime}$, that is, for $s=s(\eta), \eta>\Lambda^{-q} \mu_{1 q}^{q} r^{q}$, we have $U(P, s)$ $\cap G^{\prime} \neq \phi$. In other words, any point $P \in \bar{G}$ is at a distance $\leqslant s=s(\eta)$ from points $Q$ of $G^{\prime}$. Hence $\left|\rho v^{*}(P)-\rho v^{*}(Q)\right| \leqslant R_{0} \mu_{1}|P-Q|$ with $\left|v^{*}(Q)\right| \leqslant \Gamma$, and finally

$$
\left|\rho v^{*}(P)\right| \leqslant R_{0} \Gamma+R_{0} \mu_{1} s(\eta) \text { for all } P \in \bar{G} \text {. }
$$

Now $s(\eta)=\pi^{-1 / 2} \eta^{1 / 2}$, and the third relation (14.6) becomes

$$
R_{0} \Gamma+R_{0} \mu_{1} s(\eta)+\Lambda \leqslant \delta .
$$

Thus, for $\rho=R_{0}$, meas $G^{\prime \prime}<\eta, \eta>\Lambda^{-q} \mu_{1 q}^{q} r^{q}$, we have

$$
\begin{aligned}
& \left|\rho v^{*}+\sigma\right| \leqslant R_{0} \Gamma+R_{0} \mu_{1} \sqrt{\eta / \pi}+\Lambda \leqslant \delta \text { in } G^{\prime} \cup G^{\prime \prime}, \\
& \text { meas } G^{\prime \prime \prime} \leqslant \Lambda^{-q} \mu_{1 q}^{q} r^{q} .
\end{aligned}
$$

Hence, $g_{u}\left(\rho v^{*}+\sigma\right) \geqslant d$ in $G^{\prime} \cup G^{\prime \prime}, g_{u}\left(\rho v^{*}+\sigma\right) \geqslant-d^{\prime}$ in $G^{\prime \prime \prime}$, and $|g| \leqslant C,\left|v_{t}^{*}\right| \leqslant \mu_{1}$ a.e. in $G$. Hence

$$
\begin{aligned}
& \int_{G} g_{u}\left(\rho v^{*}+\sigma\right)\left(\rho v_{t}^{*}+\sigma_{t}\right)\left(\rho v_{t}^{*}\right) \mathrm{d} t \mathrm{~d} x \\
& =\int_{G} g_{u}(\rho v+\sigma)\left(\rho v_{t}^{*}\right)^{2} \mathrm{~d} t \mathrm{~d} x+\int_{G} g_{u}\left(\rho v^{*}+\sigma\right)\left(\rho v_{t}^{*}\right) \sigma_{t} \mathrm{~d} t \mathrm{~d} x \\
& =\int_{G^{\prime} \cup G^{\prime \prime}} g_{u}\left(\rho v^{*}+\sigma\right)\left(\rho v_{t}^{*}\right)^{2} \mathrm{~d} t \mathrm{~d} x+\int_{G^{\prime \prime}} g_{u}\left(\rho v^{*}+\sigma\right)\left(\rho v_{t}^{*}\right)^{2} \mathrm{~d} t \mathrm{~d} x \\
& +\int_{G} g_{u}\left(\rho v^{*}+\sigma\right)\left(\rho v_{t}^{*}\right) \sigma_{t} \mathrm{~d} t \mathrm{~d} x \\
& \geqslant d \int_{G}\left(\rho v_{t}^{*}\right)^{2} \mathrm{~d} t \mathrm{~d} x-d \int_{G^{\prime \prime}}\left(\rho v_{l}^{*}\right)^{2} \mathrm{~d} t \mathrm{~d} x+\int_{G^{\prime \prime \prime}} g_{u}\left(\rho v^{*}+\sigma\right)\left(\rho v_{t}^{*}\right)^{2} \mathrm{~d} t \mathrm{~d} x \\
& +\int_{G} g_{u}\left(\rho v^{*}+\sigma\right)\left(\rho v_{t}^{*}\right) \sigma_{t} \mathrm{~d} t \mathrm{~d} x \\
& \geqslant d R_{0}^{2} 2^{-1}\left(1-\lambda^{2}\right)-\left(d+d^{\prime}\right) R_{0}^{2} \mu_{1}^{2} \Lambda^{-q} \mu_{1 q}^{q} r^{q}-D R_{0} 2^{-1 / 2}\left(1-\lambda^{2}\right)^{1 / 2} r .
\end{aligned}
$$

On the other hand

$$
\begin{gathered}
\int_{G} g\left(\rho v^{*}+\sigma\right)\left(\rho v^{*}\right) \mathrm{d} t \mathrm{~d} x \geqslant-C R_{0} \mu_{10} \\
\left(g\left(\rho v^{*}+\sigma\right)_{00}\left(\rho v_{0}\right)\right) \geqslant-C R_{0} \lambda \geqslant-C R_{0} .
\end{gathered}
$$


Now let us assume meas $G^{\prime \prime} \geqslant \eta$. Then, for $\rho=R_{0}$, and $(t, x) \in G^{\prime \prime}$ we have

$$
\begin{aligned}
& \rho v^{*}+\sigma \geqslant R_{0} \Gamma-\Lambda \geqslant b \quad \text { if } v^{*} \geqslant \Gamma, \\
& \rho v^{*}+\sigma \leqslant-R_{0} \Gamma+\Lambda \leqslant-b \quad \text { if } v^{*} \leqslant-\Gamma,
\end{aligned}
$$

and in any case $g\left(\rho v^{*}+\sigma\right)\left(\rho v^{*}\right) \geqslant B R_{0} \Gamma$ in $G^{\prime \prime}-G^{\prime \prime \prime}$. Then

$$
\begin{aligned}
& \int_{G^{\prime \prime}} g\left(\rho v^{*}+\sigma\right)\left(\rho v^{*}\right) \mathrm{d} t \mathrm{~d} x \\
& \quad=\int_{G^{\prime}} g\left(\rho v^{*}+\sigma\right)\left(\rho v^{*}+\sigma\right) \mathrm{d} t \mathrm{~d} x-\int_{G^{\prime}} g\left(\rho v^{*}+\sigma\right) \sigma \mathrm{d} t \mathrm{~d} x \\
& \quad \geqslant 0-C\|\sigma\|_{L_{1}} \geqslant-C \mu_{10} r .
\end{aligned}
$$

On the other hand.

$$
\begin{aligned}
& \int_{G} g_{u}\left(\rho v^{*}+\sigma\right)\left(\rho v_{t}^{*}+\sigma_{t}\right)\left(\rho v_{t}^{*}\right) \mathrm{d} t \mathrm{~d} x \\
& \quad=\int_{G} g_{u}\left(\rho v^{*}+\sigma\right)\left(\rho v_{t}^{*}\right)^{2} \mathrm{~d} t \mathrm{~d} x+\int_{G} g_{u}\left(\rho v^{*}+\sigma\right)\left(\rho v_{t}^{*}\right) \sigma_{t} \mathrm{~d} t \mathrm{~d} x \\
& \quad \geqslant-d^{\prime} R_{0}^{2} 2^{-1}\left(1-\lambda^{2}\right)-D R_{0} 2^{-1 / 2}\left(1-\lambda^{2}\right)^{1 / 2} r
\end{aligned}
$$

Finally, if we assume $\Gamma \geqslant \mu_{1}(D / 2)=\mu_{1} \sqrt{2 \pi}$, and $v^{*}(t, x) \geqslant \Gamma$ at some point of $G^{\prime \prime}$, then $v^{*}$ $\geqslant 0$ everywhere in $G$; if $v^{*}(t, x) \leqslant-\Gamma$ at some point of $G^{\prime \prime}$, then $v^{*} \leqslant 0$ everywhere in $G$. In other words, $v^{*}$ has constant sign, and the same holds for $\rho v^{*}$, and for $g\left(\rho v^{*}\right)$. Thus, $\left(g\left(\rho v^{*}\right)\right)_{00}$ and $\left(\rho v^{*}\right)_{00}$ have the same sign. Now

$$
\begin{aligned}
& \left(g\left(\rho v^{*}+\sigma\right)\right)_{00}\left(\rho v^{*}\right)_{00}=\left(g\left(\rho v^{*}\right)\right)_{00}\left(\rho v^{*}\right)_{00}+\left[\left(g\left(\rho v^{*}+\sigma\right)\right)_{00}-\left(g\left(\rho v^{*}\right)\right)_{00}\left(\rho v^{*}\right)_{00}\right. \\
& \geqslant 0-\left(4 \pi^{2}\right)^{-1} \int_{G}\left[g\left(\rho v^{*}+\sigma\right)-g\left(\rho v^{*}\right)\right] \mathrm{d} t \mathrm{~d} x \cdot\left(4 \pi^{2}\right)^{-1} \int_{G}\left(\rho v^{*}\right) \mathrm{d} t \mathrm{~d} x \\
& \geqslant-\left(4 \pi^{2}\right)^{-2} D \int_{G}|\sigma| \mathrm{d} t \mathrm{~d} x \cdot \int_{G} \rho\left|v^{*}\right| \mathrm{d} t \mathrm{~d} x \\
& \geqslant-\left(4 \pi^{2}\right)^{-2} D \mu_{10} r \cdot R_{0} \mu_{10}=-(4 \pi)^{-2} \mu_{10}^{2} D R_{0} r .
\end{aligned}
$$

Now let us assume that $\left|v_{00}^{*}\right| \geqslant \lambda$. Then

$$
\left\|\left(v^{*}-v_{00}\right)_{t}\right\|_{L_{2}}^{2}=\left\|\left(v^{*}-v_{00}\right)_{x}\right\|_{L_{2}}^{2} \leqslant 2^{-1}\left(1-\lambda^{2}\right) .
$$

Hence

$$
\left|v^{*}-v_{00}\right| \leqslant \mu_{0} 2^{-1 / 2}\left(1-\lambda^{2}\right)^{1 / 2}(D / 2)=\pi \mu_{0}\left(1-\lambda^{2}\right)^{1 / 2} .
$$

If $\lambda \geqslant \pi \mu_{0}\left(1-\lambda^{2}\right)^{1 / 2}$, then $v^{*}$ has constant sign in $G$, and then $\rho v^{*}$ and $g\left(\rho v^{*}\right)$ have the same constant signs. Hence $\left(g\left(\rho v^{*}\right)\right)_{00}\left(\rho v^{*}\right)_{00} \geqslant 0$.

If $\lambda>\pi \mu_{0}\left(1-\lambda^{2}\right)^{1 / 2}+\tau$ for some $\tau>0$, then either $v^{*} \geqslant \tau$ in $G$, or $v^{*} \leqslant-\tau$ in $G$. Hence, for $R_{0}>b / \tau$ and $\rho=R_{0}$ we have either $\rho v^{*} \geqslant b$ or $\rho v^{*} \leqslant-b$, and correspondingly either $g\left(\rho v^{*}\right) \geqslant B$ or $g\left(\rho v^{*}\right) \leqslant-B$. Thus, in any case

$$
\left(g\left(\rho v^{*}\right)\right)_{00}\left(\rho v^{*}\right)_{00} \geqslant B R_{0} \tau
$$


and as before

$$
\left(g\left(\rho v^{*}+\sigma\right)\right)_{00}\left(\rho v^{*}\right)_{00} \geqslant B R_{0} \tau-\left(4 \pi^{2}\right)^{-1} \mu_{10}^{2} D R_{0} r
$$

On the other hand,

$$
\begin{gathered}
\int_{G} g_{u}\left(\rho v^{*}+\sigma\right)\left(\rho v_{t}^{*}+\sigma_{t}\right)\left(\rho v_{t}^{*}\right) \mathrm{d} t \mathrm{~d} x \\
=\int_{G} g_{u}\left(\rho v^{*}+\sigma\right)\left(\rho v_{t}^{*}\right)^{2} \mathrm{~d} t \mathrm{~d} x+\int_{G} g_{u}\left(\rho v^{*}+\sigma\right)\left(\rho v_{t}^{*}\right) \sigma_{t} \mathrm{~d} t \mathrm{~d} x \\
\geqslant-d^{\prime} R_{0}^{2} 2^{-1}\left(1-\lambda^{2}\right)-C R_{0} 2^{-1 / 2}\left(1-\lambda^{2}\right)^{1 / 2} r \\
\int_{G} g\left(\rho v^{*}+\sigma\right)\left(\rho v^{*}\right) \mathrm{d} t \mathrm{~d} x=\int_{G} g\left(\rho v^{*}+\sigma\right)\left(\rho v^{*}+\sigma\right) \mathrm{d} t \mathrm{~d} x-\int_{G} g\left(\rho v^{*}+\sigma\right) \sigma \mathrm{d} t \mathrm{~d} x \\
\geqslant 0-C \|_{L_{1}} \geqslant-C \mu_{10} .
\end{gathered}
$$

Thus, summarizing, we can say that for $\left|v_{00}^{*}\right| \leqslant \lambda$, meas $G^{\prime \prime}<\eta$, we have

$$
\begin{aligned}
\Omega & >\beta d R_{0}^{2} 2^{-1}\left(1-\lambda^{2}\right)-\beta\left(d+d^{\prime}\right) R_{0}^{2} \mu_{1}^{2} \Lambda^{-q} \mu_{q 1}^{q} r^{q}-\beta D R_{0} 2^{-1 / 2}\left(1-\lambda^{2}\right)^{1 / 2} r \\
& -\alpha C R_{0} \mu_{10}-\gamma C R_{0} \lambda-\gamma\left|\phi_{00}\right| \lambda-\alpha R_{0}\|\phi\|_{L_{2}} \mu_{20} 2^{-1 / 2}\left(1-\lambda^{2}\right)^{1 / 2} \\
& -\beta\left\|\phi_{t}\right\|_{L_{2}} R_{0} 2^{-1 / 2}\left(1-\lambda^{2}\right)^{1 / 2} .
\end{aligned}
$$

For $\left|v_{00}^{*}\right| \leqslant \lambda$, meas $G^{\prime \prime}>\eta$, we have

$$
\begin{aligned}
\Omega & >\alpha\left(\eta-\Lambda^{-q} \mu_{q 1}^{q} 1^{q}\right) B R_{0} \Gamma-\alpha C \mu_{0} r-\beta d^{\prime} R_{0}^{2} 2^{-1}\left(1-\lambda^{2}\right) \\
& -\beta D R_{0} 2^{-1 / 2}\left(1-\lambda^{2}\right)^{1 / 2} r-\gamma\left(4 \pi^{2}\right)^{-2} \mu_{10}^{2} D R_{0} r \\
& -\gamma\left|\phi_{00}\right| \lambda-\alpha R_{0}\|\phi\|_{L_{2}} \mu_{20} 2^{-1 / 2}\left(1-\lambda^{2}\right)^{1 / 2}-\beta\left\|\phi_{\|}\right\|_{L_{2}} R_{0} 2^{-1 / 2}\left(1-\lambda^{2}\right)^{1 / 2} .
\end{aligned}
$$

Note that $\left|v_{00}^{*}\right| \leqslant\left(4 \pi^{2}\right)^{-1} \int_{G}\left|v^{*}\right| \mathrm{d} t \mathrm{~d} x \leqslant\left(4 \pi^{2}\right)^{-1} \mu_{10} 2^{-1 / 2}$. Thus, for $\left|v_{00}^{*}\right|>\lambda$, then $\lambda<\left|v_{00}^{*}\right| \leqslant$ $\left(4 \pi^{2}\right)^{-1} \mu_{10} 2^{-1 / 2}$, and

$$
\begin{aligned}
\Omega & >\gamma B R_{0} \tau-\gamma\left(4 \pi^{2}\right)^{-1} \mu_{10}^{2} D R_{0} r-\alpha C \mu_{10} r \\
& -\beta d^{\prime} R_{0}^{2} 2^{-1}\left(1-\lambda^{2}\right)-\beta C R_{0} 2^{-1 / 2}\left(1-\lambda^{2}\right)^{1 / 2} r \\
& -\gamma\left|\phi_{00}\right|\left(2^{-1 / 2}\left(4 \pi^{2}\right)^{-1} \mu_{10}\right)-\alpha R_{0}\|\phi\|_{L_{2}} 2^{-1 / 2} \mu_{10}-\beta\left\|\phi_{\|}\right\|_{L_{2}} R_{0} 2^{-1 / 2} .
\end{aligned}
$$

Thus, $A_{0}, B_{0}, C_{0}$ are the maxima of the coefficients of $\left|\phi_{00}\right|,\|\phi\|_{L_{2}},\left\|\phi_{t}\right\|_{L_{2}}$ in the formulas above, and $M_{1}, M_{2}, M_{3}$ are the parts in these formulas independent of $\phi$. We conclude that, for

$$
A_{0}\left|\phi_{00}\right|+B_{0}\left\|\phi_{L_{2}}+C_{0}\right\| \phi_{t} \|_{L_{2}} \leqslant \min \left[M_{1}, M_{2}, M_{3}\right]
$$

we have $\Omega>0$ in all cases, and (14.7) is satisfied.

If we take $B=(1-\varepsilon) d b, C=(1+\theta) d$ for some fixed constants $0<\varepsilon<1, \theta>0$, and $d^{\prime}=k^{\prime} d, d^{\prime \prime}=k^{\prime \prime} d, D=k d, k=\max \left[k^{\prime}, k^{\prime \prime}\right]$, then the relations above yield

$$
\begin{aligned}
M_{1}= & \beta d R_{0}^{2} 2^{-1}\left(1-\lambda^{2}\right)-\beta\left(k+k^{\prime}\right) d R_{0}^{2} \mu_{1}^{2} \Lambda^{-q_{q}} \mu_{q_{q}} r^{q}-\beta k d R_{0} 2^{-1 / 2}\left(1-\lambda^{2}\right)^{1 / 2} r \\
& -\alpha(1+\theta) d \delta R_{0} \mu_{10}-\gamma(1+\theta) d \delta R_{0} \lambda,
\end{aligned}
$$




$$
\begin{aligned}
M_{2}= & \alpha\left(\eta-\Lambda^{-q} \mu_{q 1}^{q} r^{q}\right)(1-\varepsilon) d b R_{0} \Gamma-\alpha(1+\theta) d \delta \mu_{0} r-\beta k^{\prime} d R_{0}^{2} 2^{-1}\left(1-\lambda^{2}\right) \\
& -\beta k d R_{0} 2^{-1 / 2}\left(1-\lambda^{2}\right)^{1 / 2} r-\gamma\left(4 \pi^{2}\right)^{-1} \mu_{10}^{2} k d R_{0} r, \\
M_{3}= & \gamma(1-\varepsilon) R_{0} d b \tau-\gamma\left(4 \pi^{2}\right)^{-2} \mu_{10}^{2} k d R_{0} r-\alpha(1+\theta) d \delta \mu_{10} r \\
& -\beta k^{\prime} d R_{0}^{2} 2^{-1}\left(1-\lambda^{2}\right)-\beta(1+\theta) d \delta R_{0} 2^{-1 / 2}\left(1-\lambda^{2}\right)^{1 / 2} r .
\end{aligned}
$$

Thus, the inequalities (14.6) become

$$
\begin{gathered}
M_{1} d^{-1} R_{0}^{-1} 2^{-1}=\beta R_{0} 2^{-1}\left(1-\lambda^{2}\right)-\left(k+k^{\prime}\right) R_{0} \mu_{1}^{2} \Lambda^{-q} \mu_{q}^{q} r^{q} \\
-k 2^{-1 / 2}\left(1-\lambda^{2}\right)^{1 / 2} r-\alpha(1+\theta) \delta \mu_{10}-\gamma(1+\theta) \delta \lambda>0, \\
M_{2} d^{-1}=\alpha\left(\eta-\Lambda^{-q} \mu_{q 1}^{q} r^{q}\right)(1-\varepsilon) b R_{0} \Gamma-\alpha(1+\theta) \delta \mu_{0} r-\beta k^{\prime} R_{0}^{2} 2^{-1}\left(1-\lambda^{2}\right) \\
-\beta k R_{0} 2^{-1 / 2}\left(1-\lambda^{2}\right)^{1 / 2} r-\gamma\left(4 \pi^{2}\right)^{-1} \mu_{10}^{2} k R_{0} r>0, \\
M_{3} d^{-1}=\gamma(1-\varepsilon) R_{0} b \tau-\gamma\left(4 \pi^{2}\right)^{-2} \mu_{10}^{2} k R_{0} r-\alpha(1+\theta) \delta \mu_{10} r-\beta k^{\prime} R_{0}^{2} 2^{-1}\left(1-\lambda^{2}\right) \\
-\beta(1+\theta) \delta R_{0} 2^{-1 / 2}\left(1-\lambda^{2}\right)^{1 / 2} r>0, \\
2 \pi(1+\theta) d \delta<r, \quad \eta>\Lambda^{-q} \mu_{q 1}^{q} r^{q}, \\
R_{0} \Gamma+R_{0} \mu_{1} \sqrt{\eta / \pi}+\Lambda \leqslant \delta, \quad R_{0} \Gamma-\Lambda \geqslant b, \\
\Gamma>\mu_{1} \sqrt{2 \pi}, \quad 0<\eta<a=4 \pi^{2}, \\
0 \leqslant \lambda \leqslant 1, \quad \lambda \geqslant \pi \mu_{0}\left(1-\lambda^{2}\right)^{1 / 2}+\sigma, \quad R_{0} \geqslant b / \sigma .
\end{gathered}
$$

If we take $B=(1-\varepsilon) d b, C=(1+\theta) d \delta$ for some constants $0<\varepsilon<1, \theta>0$, and $d^{\prime}=k^{\prime} d$, $d^{\prime \prime}=k^{\prime \prime} d, D=k d, K=\max \left[k^{\prime}, k^{\prime \prime}\right]$, then the relations above become $M_{1}=\beta d R_{0}^{2} 2^{-1}\left(1-\lambda^{2}\right)-\beta\left(k+k^{\prime}\right) d R_{0}^{2} \mu_{1}^{2} \Lambda^{-q} \mu_{q 1}^{q} r^{q}-\beta k d R_{0} 2^{-1 / 2}\left(1-\lambda^{2}\right)^{1 / 2} r-\alpha(1+\theta) d \delta R_{0} \mu_{10}$ $-\gamma(1+\theta) d \delta R_{0} \lambda>0$, $M_{2}=\alpha\left(\eta-\Lambda^{q} \mu_{q 1}^{q} r^{q}\right)(1-\varepsilon) d b R_{0} \Gamma-\alpha(1+\theta) d \delta \mu_{0} r-\beta k^{\prime} d R_{0}^{2} 2^{-1}\left(1-\lambda^{2}\right)$

$-\beta k d R_{0} 2^{-1 / 2}\left(1-\lambda^{2}\right)^{1 / 2} r-\gamma\left(4 \pi^{2}\right)^{-1} \mu_{10}^{2} k d R_{0} r>0$,

$M_{3}=\alpha(1-\varepsilon) R_{0} d b \tau-\gamma\left(4 \pi^{2}\right)^{-2} \mu_{10}^{2} k d R_{0} r-\alpha(1+\theta) d \delta \mu_{10} r-\beta k^{\prime} d R_{0}^{2} 2^{-1}\left(1-\lambda^{2}\right)$

$$
-\beta(1+\theta) d \delta R_{0} 2^{-1 / 2}\left(1-\lambda^{2}\right)^{1 / 2} r>0 .
$$

We also have

$$
\begin{aligned}
M_{1} d^{-1} R_{0}^{-1}= & \beta R_{0} 2^{-1}\left(1-\lambda^{2}\right)-\left(k+k^{\prime}\right) R_{0} \mu_{1}^{2} \Lambda^{-q} \mu_{q} r^{q}-\beta k 2^{-1 / 2}\left(1-\lambda^{2}\right)^{1 / 2} r \\
& -\alpha(1+\lambda) \delta \mu_{10}-\gamma(1+\theta) \delta \lambda>0, \\
M_{2} d^{-1}= & \alpha\left(\eta-\Lambda^{-q} \mu_{q 1}^{q} r^{q}\right)(1-\varepsilon) b R_{0} \Gamma-\alpha(1+\theta) \delta \mu_{0} r-\beta k^{\prime} R_{0}^{2} 2^{-1}\left(1-\lambda^{2}\right) \\
& -\beta k R_{0} 2^{-1 / 2}\left(1-\lambda^{2}\right)^{1 / 2} r-\gamma\left(4 \pi^{2}\right)^{-1} \mu_{10}^{2} k R_{0} r>0,
\end{aligned}
$$




$$
\begin{gathered}
M_{3} d^{-1}=\gamma(1-\varepsilon) R_{0} b \tau-\gamma\left(4 \pi^{2}\right)^{-2} \mu_{10}^{2} k R_{0} r-\alpha(1+\theta) \delta \mu_{10} r-\beta k^{\prime} R_{0}^{2} 2^{-1}\left(1-\lambda^{2}\right) \\
\quad-\beta(1+\theta) R_{0} 2^{-1 / 2}\left(1-\lambda^{2}\right)^{1 / 2} r \delta>0, \\
2 \pi(1+\theta) d \delta<r, \quad \eta>\Lambda^{q} \mu_{q 1}^{q} r^{q}, \\
R_{0} \Gamma+R_{0} \mu_{1} \sqrt{\eta / \pi}+\Lambda \leqslant \delta, \quad R_{0} \Gamma-\Lambda \geqslant b, \\
\Gamma \geqslant \mu_{1} \sqrt{2 \pi}, \quad 0<\eta \leqslant a=4 \pi^{2}, \\
0 \leqslant \lambda \leqslant 1, \quad \lambda \geqslant \pi \mu_{0}\left(1-\lambda^{2}\right)^{1 / 2}+\tau, \quad R_{0} \geqslant b / \tau .
\end{gathered}
$$

For $\tau=0$ the equation $\lambda=\pi \mu_{0}\left(1-\lambda^{2}\right)^{1 / 2}$ yields $\lambda_{0}=\pi \mu_{0}\left(1+\left(\pi \mu_{0}\right)^{2}\right)^{-1 / 2}, 0<\lambda_{0}<1$. Then, equation $\lambda=\pi \mu_{0}\left(1-\lambda^{2}\right)^{1 / 2}+\tau$ is equivalent to $F(\lambda)=\left(1+\left(\pi \mu_{0}\right)^{2}\right) \lambda^{2}-2 \lambda \tau+\tau^{2}-\left(\pi \mu_{0}\right)^{2}=0$ with $F\left(\lambda_{0}\right)=-2 \lambda_{0} \tau+\tau^{2}$ and $F(1)=(\tau-1)^{2} \geqslant 0$. Thus, for $\tau \neq 1$, certainly $F$ has a root between $\lambda_{0}$ and 1 provided $-2 \lambda_{0} \tau+\tau^{2}<0$, or $\tau<2 \lambda_{0}$. Let us fix $\tau, 0<\tau<\min \left[1,2 \lambda_{0}\right]$, and let $\lambda$ denote the root between $\lambda_{0}$ and 1 of the equation $\lambda=\pi \mu_{0}\left(1-\lambda^{2}\right)^{1 / 2}+\tau$. Now we take $k^{\prime} \leqslant k$ hence $k^{\prime \prime}=k$, and

$$
2 k \mu_{1}^{2} \cdot \Lambda^{-q} \mu_{q}^{q} r^{q} \leqslant \beta 2^{-2}\left(1-\lambda^{2}\right)
$$

Then

$$
M_{1} d^{-1} R_{0}^{-1} \geqslant \beta 2^{-2}\left(1-\lambda^{2}\right) R_{0}-k 2^{-1 / 2}\left(1-\lambda^{2}\right)^{1 / 2} r-\alpha(1+\theta) \delta \mu_{10}-\gamma(1+\theta) \delta \lambda,
$$

and we take

so that

$$
\begin{aligned}
& k 2^{-1 / 2}\left(1-\lambda^{2}\right)^{1 / 2} r \leqslant \beta 2^{-4}\left(1-\lambda^{2}\right) R_{0}, \\
& \alpha(1+\theta) \delta \mu_{10} \leqslant \beta 2^{-4}\left(1-\lambda^{2}\right) R_{0}, \\
& \gamma(1+\theta) \delta \leqslant \beta 2^{-4}\left(1-\lambda^{2}\right) R_{0},
\end{aligned}
$$

Analogously, we take

Then

$$
M_{1} d^{-1} R_{0}^{-1} \geqslant \beta 2^{-4}\left(1-\lambda^{2}\right) R_{0}
$$

$$
\Lambda^{-q} \mu_{q 1}^{q} r^{q} \leqslant \eta / 2
$$

$$
\begin{aligned}
M_{2} d^{-1} \geqslant & {\left[\alpha(\eta / 2)(1-\varepsilon) b \Gamma-\beta k 2^{-1 / 2}\left(1-\lambda^{2}\right)^{1 / 2} r-\gamma\left(4 \pi^{2}\right)^{-1} \mu_{10}^{2} k r\right] R_{0} } \\
& -\alpha(1+\theta) \delta \mu_{0} r-\beta k^{\prime} R_{0}^{2} 2^{-1}\left(1-\lambda^{2}\right)
\end{aligned}
$$

and we take

$$
\begin{aligned}
& \beta k_{2}^{-1 / 2}\left(1-\lambda^{2}\right)^{1 / 2} r \leqslant 2^{-3} \alpha \eta(1-\varepsilon) b \Gamma \\
& \gamma\left(4 \pi^{2}\right)^{-1} \mu_{10}^{2} k r \leqslant 2^{-3} \alpha \eta(1-\varepsilon) b \Gamma \\
& \alpha(1+\theta) \delta \mu_{0} r \leqslant 2^{-4} \alpha \eta(1-\varepsilon) \Gamma R_{0} \\
& \beta k^{\prime} R_{0}^{2} 2^{-1}\left(1-\lambda^{2}\right) \leqslant 2^{-4} \alpha \eta(1-\varepsilon) \Gamma R_{0}
\end{aligned}
$$

so that

$$
M_{2} d^{-1} \geqslant 2^{-3} \alpha \eta(1-\varepsilon) \Gamma R_{0}
$$

Analogously,

$$
\begin{aligned}
M_{3} d^{-1}= & {\left[\gamma(1-\varepsilon) b \tau-\gamma\left(4 \pi^{2}\right)^{-2} \mu_{10}^{2} k r-\beta(1+\theta) \delta 2^{-1 / 2}\left(1-\lambda^{2}\right)^{1 / 2} r\right] R_{0} } \\
& -\alpha(1+\theta) \delta \mu_{10} r-\beta k^{\prime} R_{0}^{2} 2^{-1}\left(1-\lambda^{2}\right),
\end{aligned}
$$


and we take

$$
\begin{aligned}
& \gamma\left(4 \pi^{2}\right)^{-2} \mu_{10}^{2} k r \leqslant 2^{-2} \gamma(1-\varepsilon) b \tau \\
& \beta(1+\theta) \delta 2^{-1 / 2}\left(1-\lambda^{2}\right)^{1 / 2} r \leqslant 2^{-2} \gamma(1-\varepsilon) b \tau, \\
& \alpha(1+\theta) \delta \mu_{10} r \leqslant 2^{-3} \gamma(1-\varepsilon) b \tau R_{0} \\
& \beta k^{\prime} R_{0}^{2} 2^{-1}\left(1-\lambda^{2}\right) \leqslant 2^{-3} \gamma(1-\varepsilon) b \tau R_{0},
\end{aligned}
$$

so that

$$
M_{3} d^{-1} \geqslant 2^{-1} \gamma(1-\varepsilon) b \tau R_{0} .
$$

We think of $\alpha, \beta, \gamma, \varepsilon, \theta, \tau, \lambda$ as fixed numbers.

We write the second relation (14.9) in the form

$$
b+\Lambda \leqslant R_{0} \Gamma \leqslant \varepsilon-\Lambda-R_{0} \mu_{1} \sqrt{\eta / \pi}
$$

so that we must arrange that the first member is $\leqslant$ the third member, or

$$
2 \Lambda+R_{0} \mu_{1} \sqrt{\eta / \pi} \leqslant \delta-b .
$$

Let us take $\Gamma>\mu_{1} \sqrt{2 \pi}$ and $0<\eta<4 \pi^{2}$ arbitrarily; in other words we have satisfied the third relations (14.9).

Now equations (14.12) and (14.13) can be written in the form

$$
b / \tau \leqslant R_{0}, \quad \frac{\alpha}{\beta} \frac{(1+\theta) \mu_{10}}{2^{-4}\left(1-\lambda^{2}\right)} \delta \leqslant R_{0}, \quad \frac{\gamma}{\beta} \frac{1+\theta}{2^{-4}\left(1-\lambda^{2}\right)} \delta \leqslant R_{0},
$$

and these relations together with (14.23) yields

$$
\begin{aligned}
& \frac{\alpha}{\beta} \frac{(1+\theta) \mu_{10}}{2^{-4}\left(1-\lambda^{2}\right)} \mu_{1} \sqrt{\frac{\eta}{\pi}} \delta \leqslant \mu_{1} \sqrt{\frac{\eta}{\pi}} R_{0} \leqslant \delta-b-2 \Lambda, \\
& \frac{\gamma}{\beta} \frac{1+\theta}{2^{-4}\left(1-\lambda^{2}\right)} \mu_{1} \sqrt{\frac{\eta}{\pi}} \delta \leqslant \mu_{1} \sqrt{\frac{\eta}{\pi}} R_{0} \leqslant \delta-b-2 \Lambda .
\end{aligned}
$$

Thus, we must require that

$$
\frac{\alpha}{\beta} \frac{(1+\theta) \mu_{10}}{2^{-4}\left(1-\lambda^{2}\right)} \mu_{1} \sqrt{\frac{\eta}{\pi}}<1, \quad \frac{\gamma}{\beta} \frac{1+\theta}{2^{-4}\left(1-\lambda^{2}\right)} \mu_{1} \sqrt{\frac{\eta}{\pi}}<1,
$$

and these relations can be satisfied by taking $\alpha, \beta, \gamma>0$ with $\alpha$ and $\gamma$ sufficiently small with respect to $\beta$. Actually, we shall choose $\alpha, \rho, \gamma$ so that, if $\zeta$ is the larger of the two numbers in the first members of $(14.24)$, we have

$$
\zeta \leqslant\left(\mu_{1} \sqrt{\eta / \pi}\right)\left(\tau+\mu_{1} \sqrt{\eta / \pi}\right)^{-1}<1,
$$

hence, $\zeta\left(\mu_{1} \sqrt{\eta / \pi}\right)^{-1}<\left(\tau+\mu_{1} \sqrt{\eta / \pi}\right)^{-1}$. Then, we take $\delta>0$ arbitrary, and

$$
\zeta\left(\mu_{1} \sqrt{\eta / \pi}\right)^{-1} \delta \leqslant R_{0}<\left(\tau+\mu_{1} \sqrt{\eta / \pi}\right)^{-1} \delta, \quad b=\tau R_{0}, \quad 0<\Lambda<2^{-1}\left[\delta-\left(\tau+\mu_{1} \sqrt{\eta / \pi}\right)\right] R_{0} .
$$

Then, we have

$$
(\alpha / \beta)(1+\theta) \mu_{10} 2^{4}\left(1-\lambda^{2}\right)^{-1} \delta \leqslant \zeta\left(\mu_{1} \sqrt{\eta / \pi}\right)^{-1} \delta \leqslant R_{0}
$$




$$
\begin{aligned}
& (\gamma / \beta)(1+\theta) 2^{4}\left(1-\lambda^{2}\right)^{-1} \delta \leqslant \zeta\left(\mu_{1} \sqrt{\eta / \pi}\right)^{-1} \delta \leqslant R_{0}, \\
& 2 \Lambda+R_{0} \mu_{1} \sqrt{\eta / \pi}+b \leqslant \delta-\left(\tau+\mu_{1} \sqrt{\eta / \pi}\right) R_{0}+R_{0} \mu_{1} \sqrt{\eta / \pi}+\tau R_{0}=\delta .
\end{aligned}
$$

With the choice we have made of $\alpha, \beta, \gamma, \delta, R_{0}, b, \Lambda$, relations (14.12), (14.13) and (14.23) are satisfied.

Now relations (14.11), (14.12), (14.14), (14.15), (14.16), (14.17), (14.19), (14.20), (14.21) and second relation in the first line of (14.9) can be used to determine $r>0$. Then the first relation in the first line of (14.9) can be used to determine $d>0$. Finally, equation (14.18) can be used to determine $k^{\prime}$.

We have shown that the inequalities we have required are compatible.

\section{APPENDIX}

1. Let us consider the problem of the doubly $2 \pi$-periodic solutions $u(t, x)$ of the hyperbolic problem

$$
\pm \varepsilon u+u_{n}-u_{x x}=f(t, x, u),(t, x) \in \mathbb{R}^{2},
$$

where $f(t, x, u)$ is a double $2 \pi$-periodic in $t, x$, continuous in $u$ for all $t, x$, measurable in $(t, x)$ for all $u$, and

$$
|f(t, x, u)| \leqslant f_{0}(t, x)+h(|u|)
$$

with $f_{0}(t, x) \geqslant 0$ a fixed doubly $2 \pi$-periodic function in $(t, x), f_{0} \in L_{2}(G)$, and $h(\xi) \geqslant 0,0 \leqslant \xi<+\infty$, is a monotone nondecreasing function with $h(\xi) / \xi \rightarrow 0$ as $\xi \rightarrow+\infty$.

(i) Problem (A1) has always a weak solution $u(t, x), u \in L_{2}(G)$, for $\varepsilon>0$ sufficiently small.

Proof. By the notation of section 14 , for $u \in L_{2}(G)$, then

$$
\begin{gathered}
u(t, x)=\sum_{k, l} b_{k} e_{k l}, \quad b_{k l}=\left(u, e_{k l}\right), \quad \sum_{k, l} b_{k l}^{2}=\|u\|_{L_{2}}^{2}, \\
(I-Q) u(t, x)=\sum_{k^{2} \neq l^{2}} b_{k l} e_{k l}, \\
w(t, x)=H(I-Q)=\sum_{k^{2} \neq l^{2}}\left(-k^{2}+l^{2}\right)^{-1} b_{k l} e_{k l}, \\
w \in L_{2}(G), \quad\|w\|_{L_{2}} \leqslant\|u\|_{L 2},
\end{gathered}
$$

and we take

$$
N u=f(t, x, u(t, x)) \mp a u(t, x), \quad E u=u_{n}-u_{x x}
$$

We take $X=Y=L_{2}(G), P=Q, I-P=I-Q$. We define $X_{0}, X_{1}, Y_{0}, Y_{1}$ as usual, $X_{0}=Y_{0}, X_{1}=Y_{1}$, so that $H(I-Q): Y_{1} \rightarrow X_{1}$ has norm $L=1$.

For every $n$ we define the spaces $X_{o n}, X_{i n}, Y_{o n}, Y_{l n}$, we take for $\alpha_{n}: Y_{o n} \rightarrow X_{o n}$ the identity, and $R_{n}, S_{n}, S_{n}^{\prime}$ have the usual definition.

Now the original problem $E u=N u$ with $u=u^{*}+u_{1}, u^{*} \in X_{0}, u_{1} \in X_{1}$, becomes

$$
u_{1}=H(I-Q) N u, \quad Q N u=0, \quad u \in X .
$$

For every $n$ we have now the partial problem

$$
u_{1}=R_{n} H(I-Q) N u, \quad S_{n}^{\prime} Q N u=0, \quad u \in X_{n},
$$

and we consider the transformation $T_{n}$ :

$$
\begin{aligned}
& T_{n}: \begin{array}{l}
\bar{u}_{1}=R_{n} H(I-Q) N\left(u^{*}+u_{1}\right), \\
\bar{u}^{*}=u_{0}-\alpha_{n} S_{n}^{\prime} Q N\left(u^{*}+u_{1}\right),
\end{array} \\
& u=u^{*}+u_{1}, \bar{u}=\bar{u}^{*}+\bar{u}_{1}, u^{*}, \bar{u}^{*} \in X_{0}, u_{1}, \bar{u}_{1} \in X_{1}
\end{aligned}
$$

Now we restrict $u=u^{*}+u_{1}$ to the set

$$
\Sigma_{n}=\left[u=u^{*}+u_{1}, u^{*} \in X_{o n}, u_{1} \in X_{i n},\left\|u^{*}\right\| \leqslant R_{0} .\left\|u_{1}\right\| \leqslant r\right]
$$

so that $\|u\| \leqslant\left(R_{0}^{2}+r^{2}\right) 1 / 2=R$ for all $u \in \Sigma_{n}$. 
Because of $|f(t, x, u)| \leqslant f_{0}(t, x)+h(|u|)$ with $h(\xi) / \xi \rightarrow 0$ as $\xi \rightarrow+\infty$, there exists also another function $k(\xi)$, $0 \leqslant \xi \leqslant+\infty, k$ monotone nondecreasing such that, for every $u \in L_{2}(G)$ we also have (see below)

$$
\|f(t, x, u)\| \leqslant k(\|u\|)
$$

Now for $u=\rho v+\sigma, v \in X_{0},\|v\|_{L_{2}}=1, \sigma \in X_{1},\|\sigma\|_{L_{2}} \leqslant r$, and $\rho>0$ we have $\|u\|^{2}=\|\rho v+\sigma\|^{2}=\rho^{2}\|v\|^{2}+\|\sigma\|^{2}$, and

$$
\begin{aligned}
\left\|u_{1}\right\|_{L_{2}} & =\|H(I-Q) N u\| \\
& \leqslant \| H(I-Q)[\mp \varepsilon u+f(t, x, u(t, x))] \\
& \leqslant \varepsilon\|u\|+k(\|u\|) \\
= & \varepsilon\left(\rho^{2}\|v\|^{2}+\|\sigma\|^{2}\right)^{1 / 2}+k\left(\left(\rho^{2}\|v\|^{2}+\|\sigma\|^{2}\right)^{1 / 2}\right) \\
& \leqslant \varepsilon\left(\rho^{2}+r^{2}\right)^{1 / 2}+k\left(\left(\rho^{2}+r^{2}\right)^{1 / 2}\right) .
\end{aligned}
$$

For $\rho \geqslant 3^{-1 / 2} r$, and $k(2 \rho) / 2 \rho<\varepsilon$ we also have

$$
\left\|u_{1}\right\|_{L_{2}} \leqslant 2 \varepsilon \rho+k(2 \rho)=2\left(\varepsilon+\frac{k(2 \rho)}{2 \rho}\right) \rho \leqslant 4 \varepsilon \rho
$$

Finally, for $\rho \leqslant R_{0}$, and $\varepsilon<4^{-1} R_{0}^{-1} r$, we have $\left\|u_{1}\right\| \leqslant r$.

It remains to prove (A3). It is enough to prove that, given $\eta>0$, there is $N=N(\eta)>0$ such that $\|f(t, u, u(t, x))\|_{L_{2}} \leqslant \eta\|u\|_{L_{2}}$ for all $u \in L_{2}(G)$ with $\|u\| \geqslant N$. Let $\gamma>0$ be any constant, and let $N>0$ be such that $h(\xi) \leqslant \gamma \xi$ for all $\xi \geqslant N$. Given $u \in L_{2}$, let $\mu=\|u\|_{L_{2}}$ and let $\Sigma_{1}, \Sigma_{2}$ denote the sets of all $(t, x) \in G$ where $|u(t, x)| \leqslant N$ and $|u(t, x)|>N$ respectively. Then for $a=$ meas $G=4 \pi^{2}$, we have

$$
\begin{aligned}
\int_{G}(f(t, x, u(t, x)))^{2} \mathrm{~d} t \mathrm{~d} x & =\int_{G}\left(f_{0}+h(|u|)\right)^{2} \mathrm{~d} t \mathrm{~d} x \leqslant 2 \int_{G} f_{0}^{2} \mathrm{~d} t \mathrm{~d} x+2 \int_{G} h^{2}(|u|) \mathrm{d} t \mathrm{~d} x \\
& =2\left\|f_{0}\right\|_{L_{2}}^{2}+2\left(\int_{\Sigma_{1}}+\int_{\Sigma_{2}}\right) h^{2}(|u|) \mathrm{d} t \mathrm{~d} x \\
& \leqslant 2\left\|f_{0}\right\|^{2}+2 \int_{\Sigma_{1}} h^{2}(N) \mathrm{d} t \mathrm{~d} x+2 \gamma^{2} \int_{\Sigma_{2}}|u|^{2} \mathrm{~d} t \mathrm{~d} x \\
& \leqslant 2\left\|f_{0}\right\|^{2}+2 a h^{2}(N)+2 \gamma^{2} \mu^{2} .
\end{aligned}
$$

Now, for $\mu \geqslant\left(2\left\|h_{0}\right\|^{2}+2 a h^{2}(N)\right)^{1 / 2} 2^{-1 / 2} \gamma^{-1}$ we also have

$$
\|f(t, x, u(t, u))\|_{L_{2}}^{z} \leqslant 4 \gamma^{2} \mu^{2}
$$

and for $\gamma=\eta / 2$ we also have $\|f\|_{L_{2}} \leqslant \eta \mu=\eta\|u\|_{L_{2}}$.

Now we have to prove that $(N(\rho v+\sigma), v) \geqslant 0$ [or $\leqslant 0$ ] for all $v \in X_{0}, \sigma \in X_{1},\|v\|=1,\|\sigma\| \leqslant r, \rho \geqslant R_{0}$ and $R_{0}$ sufficiently large. Assume the sign minus holds in (A1). In the opposite case the argument is analogous. Then

$$
\begin{gathered}
(N(\rho v+\sigma), \rho v)=\int_{G}[\varepsilon(\rho v+\sigma)+h(t, x, \rho v+\sigma)] \rho v \mathrm{~d} t \\
\geqslant \varepsilon \rho^{2}\|v\|^{2}-\varepsilon \rho\|v\|\|\sigma\|-\rho\|v\| k(\|\rho v+\sigma\|) \\
\geqslant \varepsilon \rho^{2}\|v\|^{2}-\varepsilon \rho\|v\|\|\sigma\|-\rho\|v\| k(\rho\|v\|+\|\sigma\|)
\end{gathered}
$$

where $\|v\|=1$, $\|\sigma\| \leqslant r$. If we take $\rho \geqslant r$, then

$$
\begin{gathered}
(N(\rho v+\sigma), \rho v) \geqslant \varepsilon \rho^{2}-\varepsilon \rho r-\frac{k(2 \rho)}{2 \rho} 2 \rho^{2} \\
=\left(\varepsilon-\frac{k(2 \rho)}{2 \rho} 2\right) \rho^{2}-\varepsilon \rho r .
\end{gathered}
$$

For $\rho \geqslant R_{0}$ and $R_{0} \geqslant 2 r$ chosen so that $k(2 \xi) / 2 \xi \leqslant \varepsilon / 4$ for all $\xi \geqslant R_{0}$ we have $(N(\rho v+\sigma), \rho v) \geqslant(\varepsilon / 2) \rho^{2}-\varepsilon \rho r \geqslant 0$ for $\rho \geqslant R_{0} \geqslant 2 r$.

This proves that for every $n$ there is a fixed point $u_{n}=T_{n} u_{n}$, with $u_{n} \in \Sigma_{n}$, hence $\left\|u_{n}\right\|_{L 2} \leqslant\left(R_{0}^{2}+r^{2}\right)^{1 / 2}$ independently of $n$. Thus, there is a subsequence, say still $n$, such that $u_{n}$ converges weakly in $L_{2}$ toward a function $u \in L_{2}$ which is a solution of (A2) and a weak solution of (A1).

In particular we have proved also

(ii) If $g: \mathbb{R} \rightarrow \mathbb{R}$ is any continuous function such that $g(\xi) / \xi \rightarrow 0$ as $\xi \rightarrow \infty$, and $\phi: \mathbb{R}^{2} \rightarrow \mathbb{R}$ is any given doubly $2 \pi$ periodic function, then for $\varepsilon>0$ sufficiently small, both equations

have at least one doubly $2 \pi$-periodic solution $u(t, x) \in L_{2}(G)$.

$$
\mp \varepsilon u+u_{u}-u_{x x}=\phi(t, x)+g(u)
$$


Recently, H. Brezis [Proc. Amer. Math. Soc. Symposium on the mathematical heritage of H. Poincaré] has investigated the passage to the limit as $\varepsilon \rightarrow 0$, obtaining a solution $u(t, x)$ of the equation $u_{t t}-u_{x x}=\phi(t, x)+g(u)$.

We shall return again to this point.

2. The same identical argument applies to, and the same conclusions (i), (ii) hold for the problem

$$
\begin{array}{ll}
u_{r}-u_{x x}=f(t, x, u) & {[0, \pi] \times \mathbb{R},} \\
u(t, 0)=u(t, \pi)=0, & u(t+2 \pi, x)=u(t, x),
\end{array}
$$

as well as for the problem

$$
\begin{aligned}
& u_{\pi t}+u_{x x x x}=f(t, x, u) \\
& u(t, 0)=u_{x x}(t, 0)=u(t, \pi)=u_{x x}(t, \pi)-0 .
\end{aligned}
$$

\section{REFERENCES}

1. Adams R. A., Sobolev Spaces. Academic Press, New York (1975).

2. Bachman G. \& Narici L, Functional Analysis. Academic Press, New York (1966).

3. ByRd P. F. \& FRIEdman M. D., Handbook of Elliptic Integrals for Engineers and Scientists, 2nd edn. Springer Verlag, Berlin (1971).

4. CESARI L., Functional analysis, nonlinear differential equations, and the alternative method, in Nonlinear Functional Analysis and Differential Equations (Edited by L. Cesari, R. Kannan and J. D. Schuur), pp. 1-197. M. Dekker, New York (1976).

5. CESARI L., An abstract existence theorem across a point of resonance, Dynamical Systems, an International Symposium at the Univ. of Florida (Edited by A. R. Bednarek and L. Cesari), pp. 11-26. Academic Press, New York (1977).

6. CESARI L., Nonlinear oscillations across a point of resonance for nonselfadjoint systems, $J$. diff. Eqns 2, 43-59 (1978).

7. Cesari L. \& Kannan R., Periodic solutions of nonlinear wave equations, Archs ration. Mech. Analysis (to appear).

8. CESARI L., Existence in the large of periodic solutions of hyperbolic partial differential equations, Archs Ration. Mech. Analysis 20, 170-190 (1965).

9. CESARI L., The implicit function theorem in functional analysis, Duke math. J. 33, 417-440 (1966).

10. Cesari L. \& KanNan R., Functional analysis and nonlinear differential equations, Bull. Am. Math. Soc. 79, 1216-1219 (1973).

11. Cesari L. \& Kannan R., An abstract existence theorem at resonance, Proc. Am. math. Soc. 63, 221-225 (1977).

12. CESARI L. \& KanNAN R., Existence of solutions of nonlinear hyperbolic equations, Annali Scuola Norm. Sup., Pisa 6(4), 573-592 (1979).

13. Dieudonné J. P., Foundations of Modern Analysis. Academic Press, New York (1960).

14. FucIK S. \& MAWHIN J., Generalized periodic solutions of nonlinear telegraph equations, Nonlinear Analysis TMA 2, 609-617 (1978).

15. Hale J. K., Periodic solutions of a class of hyperbolic equations, Archs. ration. Mech. Analysis 23, 380-398 (1967).

16. Hall W. S., On the existence of periodic solutions for the equation $D_{r} u+(-1)^{p} D_{x}^{2 p} u f(\cdot, \cdot, u), J$. diff. Eqns 7, 509-526 (1970).

17. HaLL W. S., Periodic solutions of a class of weakly nonlinear evolution equations, Archs. ration. Mech. Analysis 39, 294-322 (1970).

18. HobSON E. W., The Theory of Functions of a Real Variable, 2 vols. Harren Press, Washington, D.C. (1950).

19. Kannan R. \& MCKEnNa P. J., An existence theorem by alternative method for semilinear abstract equations, Boll. Un. mat. Ital. 14-A, (5), 355-358 (1977).

20. Petzeltova H., Periodic solutions of the equation $u_{t t}+u_{x x x x}=f\left(\cdot \cdot, u, u_{t}\right)$, Czech. math. J. 23, (98), 269-285 (1973).

21. Rothe E. H., Zur Theory der topologischen Ordnung und der Vektorfelder in Banach Raumen, Composition math. 5, 177-197 (1937).

22. Rothe E. H., The theory of the topological order in some linear topological spaces, Iowa State College Journal of Science 13, 373-390 (1939).

23. RotHE E. H., On the Cesari index and the Browder-Petryshyn degree, Dynamical Systems, an International Symposium at the University of Florida. (Edited by A. R. Bednarek and L. Cesari), pp. 295-312. Academic Press, New York (1977).

24. SChwartz J. T., Nonlinear Functional Analysis. Gordon and Breach, New York (1969). 